\title{
Beam Characterization at the Neutron Radiography Reactor
}

Sarah W. Morgan

Jeffrey C. King

January 2013

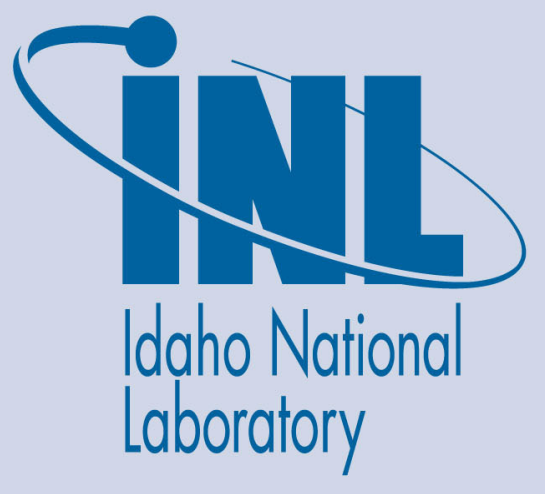

The INL is a U.S. Department of Energy National Laboratory operated by Battelle Energy Alliance 
INL/EXT-13-28427

\title{
Beam Characterization at the Neutron Radiography Reactor
}

\author{
Sarah W. Morgan ${ }^{1}$ \\ Jeffrey C. King ${ }^{1}$
}

January 2013

\section{Idaho National Laboratory \\ Idaho Falls, Idaho 83415}

http://www.inl.gov

Prepared for the U.S. Department of Energy Office of Nuclear Energy and 


\title{
BEAM CHARACTERIZATION AT THE NEUTRON RADIOGRAPHY REACTOR
}

\author{
Final Report
}

Sarah W. Morgan and Jeffrey C. King

CSM Proposal Number: 10087

CSM Index Number: 400278

INL Grant Number: 00108168 


\begin{abstract}
The quality of a neutron imaging beam directly impacts the quality of radiographic images produced using that beam. Fully characterizing a neutron beam, including determination of the beam's effective length-to-diameter ratio, neutron flux profile, energy spectrum, image quality, and beam divergence, is vital for producing quality radiographic images. This project characterized the east neutron imaging beamline at the Idaho National Laboratory Neutron Radiography Reactor (NRAD). The experiments which measured the beam's effective length-todiameter ratio and image quality are based on American Society for Testing and Materials (ASTM) standards. An analysis of the image produced by a calibrated phantom measured the beam divergence. The energy spectrum measurements consist of a series of foil irradiations using a selection of activation foils, compared to the results produced by a Monte Carlo $\mathrm{n}$ Particle (MCNP) model of the beamline. Improvement of the existing NRAD MCNP beamline model includes validation of the model's energy spectrum and the development of enhanced image simulation methods. The image simulation methods predict the radiographic image of an object based on the foil reaction rate data obtained by placing a model of the object in front of the image plane in an MCNP beamline model.
\end{abstract}




\section{TABLE OF CONTENTS}

LIST OF FIGURES FIST)

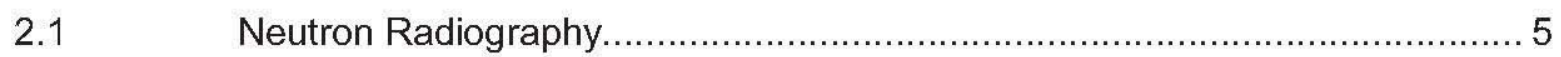

2.1.1 Neutron Radiography Reactor .................................................. 6

2.1.2 Neutron Radiography Conversion Process ........................................ 9

2.1.3 Film Processing and Optical Density ........................................... 10

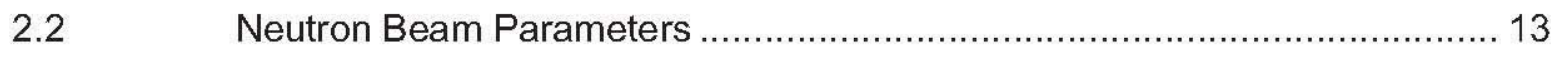

2.2.1 Effective Collimation Ratio ............................................................ 13

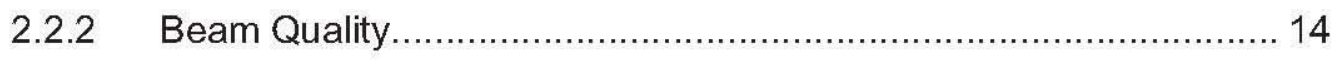

2.2.3 Beam Divergence....................................................................... 19

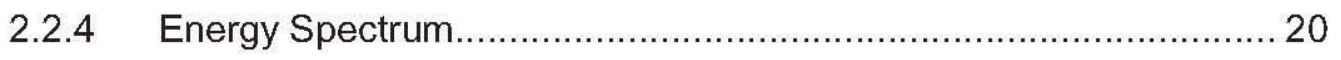

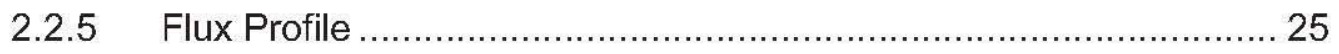

CHAPTER 3 CHARACTERIZATION EXPERIMENTS RESULTS …............................... 26

3.1 Effective Collimation Ratio Measurement ……………............................. 26

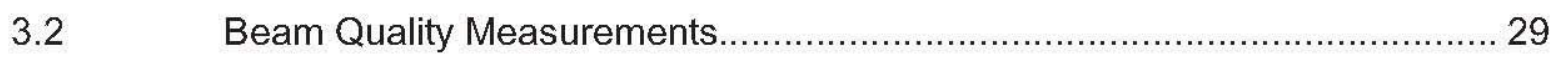

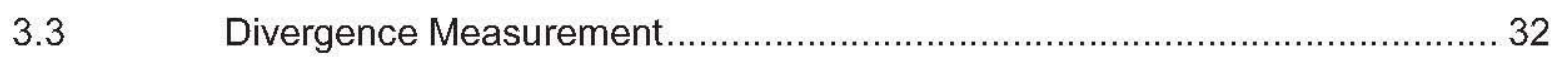

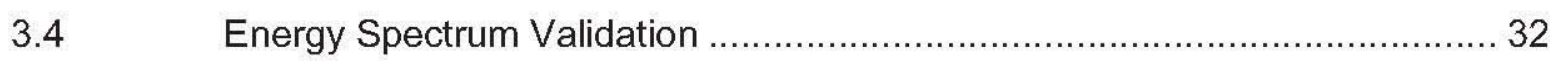

3.4.1 Bare Foil Results ........................................................................ 34

3.4.2 Cadmium Covered Foil Results ………………............................ 36 


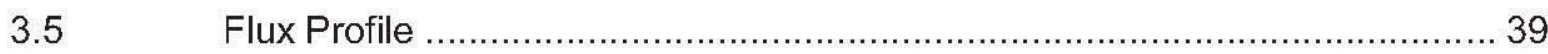

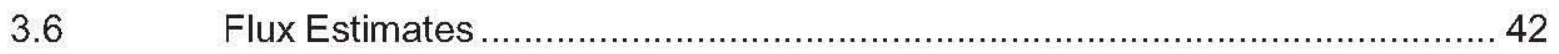

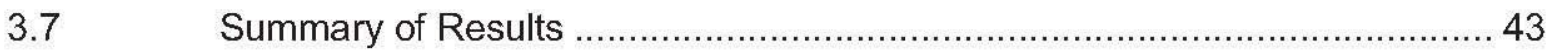

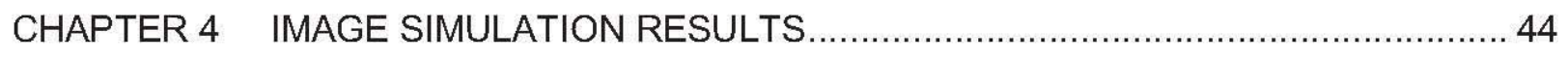

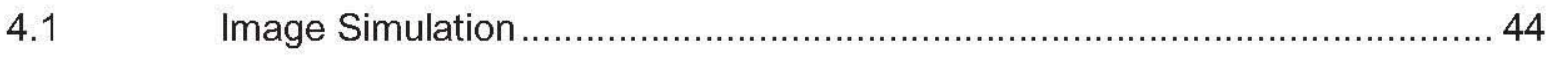

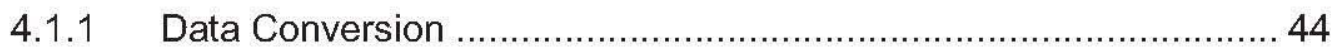

4.1.2 Development of a Film Characteristic Curve................................... 46

4.1.3 Implementation of the Simulation ................................................. 47

4.1.4 Image Simulation Validation ....................................................... 49

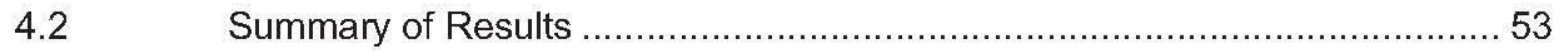

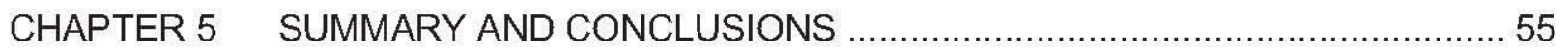

CHAPTER 6 SUGGESTIONS FOR FUTURE RESEARCH ......................................... 58

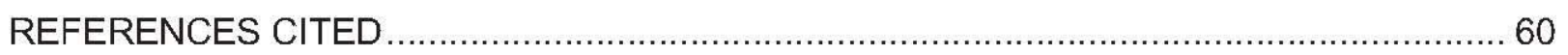

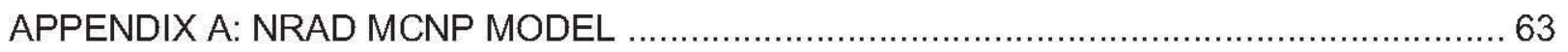

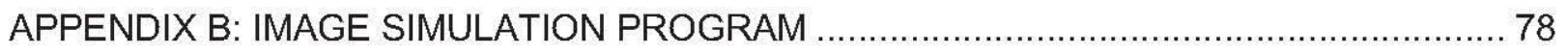




\section{LIST OF FIGURES}

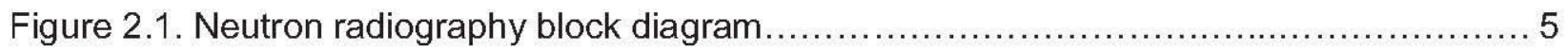

Figure 2.2. Schematic of the NRAD neutron radiography facility .........................................

Figure 2.3. NRAD beamline aperture mechanism ....................................................

Figure 2.4. D-section of the NRAD sample elevator showing foil cassette and platen ...............8

Figure 2.5. Characteristic curve for AGFA D3 s.c. X-ray film (GE Sensing and

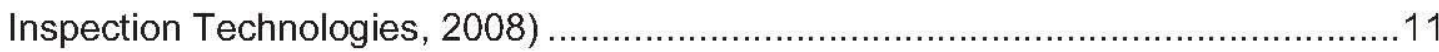

Figure 2.6. Characteristic curve for Kodak Industrex T-200 X-ray film (Carestream, 2010) .......12

Figure 2.7. Rendered view of a no-umbra device ................................................................ 15

Figure 2.8. Umbra and penumbra example .......................................................... 15

Figure 2.9. Conceptual diagram of the no-umbra device .......................................... 15

Figure 2.10. Views of the neutron radiography sensitivity indicator ..................................17

Figure 2.11. Rendering of the neutron radiography beam purity indicator .............................17

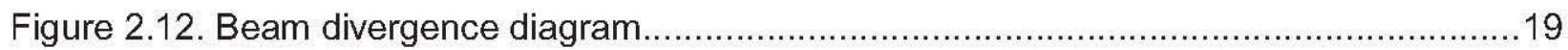

Figure 2.13. Foil activation energy ranges over-layed on a typical light water reactor spectrum taken from Malkawi and Ahmed (2000) ............................................22

Figure 2.14. Geometry of the NRAD beamline model....................................................23

Figure 2.15. Neutron energy spectrum used in the NRAD beamline model ...........................23

Figure 3.1. Renderings of the no-umbra device carrier................................................27

Figure 3.2. Renderings of the original and modified no-umbra device ...............................27

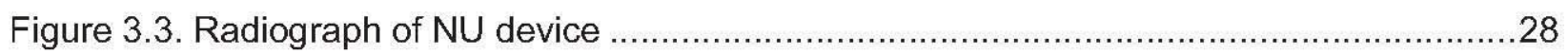

Figure 3.4. Enlarged and rotated image of the cadmium wires in the NU device ....................28

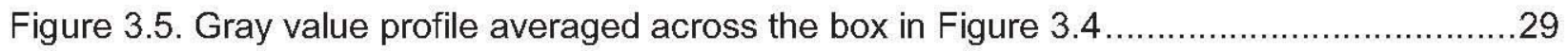

Figure 3.6. Resolution Test Piece carrier in the HFEF hot cell ........................................30

Figure 3.7. Radiograph of the beam purity and sensitivity indicators, and the divergence phantom mounted on the resolution test piece carrier ..........................30

Figure 3.8. Radiographs of sensitivity and beam purity indicators ..................................... 31

Figure 3.9. Schematic of the measured divergence phantom diameter and the actual

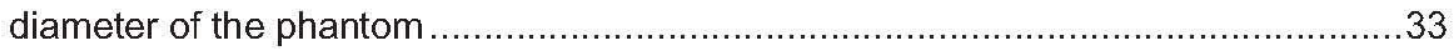

Figure 3.10. Calculated source particle rate for the bare foils ...........................................36

Figure 3.11. Calculated source particle rates for the cadmium covered foils irradiated on July 31,2011 
Figure 3.12. Calculated source particle rates for cadmium covered foils irradiated

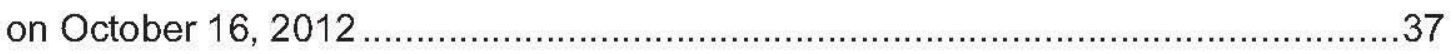

Figure 3.13. Blank indium and dysprosium radiographs ...............................................40

Figure 3.14. Normalized gray values across the dysprosium and indium foil radiographs .........41

Figure 3.15. Positions of gold and dysprosium foils on the image plane ...............................41

Figure 4.1. Grayscale image calculated by the NRAD MCNP beamline model.......................45

Figure 4.2. Flux profile of a simulated image compared to actual radiographs .......................46

Figure 4.3. Characteristic curve for AGFA D3 s.c. film exposed by activated dysprosium foil ....47

Figure 4.4. Calibration curve for Fuji FineScan 1500 scanner and an Agfa DenStep calibrated optical density step wedge 49

Figure 4.5. Polyethylene step block used to validate the image simulation program ...............50

Figure 4.6. Scanned radiograph of the polyethylene step block shown in Figure 4.5..............51

Figure 4.7. Simulated radiographs of the polyethylene step block in Figures 4.5 and 4.6 with and without the corrections for the film characteristic and scanner calibration curves.....

Figure 4.8. Averaging regions for the image data in Tables 4.2 and 4.3. .53 


\section{LIST OF TABLES}

Table 2.1. Values of characterization parameters corresponding to radiographic category

(ASTM International, 2005)

Table 2.2. Selected foils, reactions of interest, activation energy ranges, and half-lives

Table 2.3. NRAD MCNP model bin energies and probabilities .24

Table 3.1. Radiographic category designation of the NRAD 32

Table 3.2. Activation foil masses and post-irradiation activities 34

Table 3.3. Reaction rates, fluxes, and source particle rates predicted by MCNP for the bare foils. .35

Table 3.4. Cadmium covered foil fluxes and source particle rates .38

Table 3.5. MCNP calculated fluxes and fluxes calculated from foil activity. 42

Table 3.6. Thermal, resonance, and fast fluxes predicted by the MCNP model 43

Table 4.1. Irradiation results used to produce the characteristic curve in Figure 4.3 47

Table 4.2. Optical density values for the polyethylene step block in Figures 4.6and 4.7 .52

Table 4.3. Pixel values for the polyethylene step block in Figures 4.6 and 4.7 . .52 


\section{LIST OF SYMBOLS}

\section{Latin Symbol}

\section{A}

b

$\mathrm{D}_{\mathrm{H}}$

$D_{L}$ lower

$D_{L}$ higher

$D_{B}$ lower

$D_{B}$ higher

$\mathrm{D}_{\mathrm{T}}$

d

IF

L/D

m

MW

$\mathrm{N}$

$\mathrm{N}_{\mathrm{A}}$

OD

$\mathrm{R}$

$r_{1}$

$r_{2}$

$\mathrm{t}$

$t_{\text {travel }}$

$\mathrm{t}_{\text {shot }}$

SPR

$V_{\text {foil }}$

vol

w

\section{Description}

activity

"no umbra" distance

optical density of beam purity indicator (BPI) hole

optical density of lower BPI lead disc

optical density of higher BPI lead disc

optical density of lower BPI boron nitride disc

optical density of higher BPI boron nitride disc

optical density of BPI polytetrafluoroethylene block

distance from radiographed object to foil

isotope fraction

effective collimation ratio

foil mass

molar weight

number density

Avogadro's number

optical density

volumetric reaction rate

radius of phantom

radius of phantom image

irradiation time

total time between end of shot and beginning of exposure

total shot time

source particle rate

activation foil volume

voxel volume

diameter of wire in the no umbra device 


$\begin{array}{ll}\text { Greek Symbol } & \text { Description } \\ \varphi & \text { neutron flux } \\ \lambda & \text { decay constant } \\ \sigma & \text { microscopic absorption cross section } \\ \theta & \text { beam divergence angle }\end{array}$




\section{CHAPTER 1}

\section{INTRODUCTION}

There are many ways to characterize a neutron beam. The most common quantities measured for radiography facilities are the neutron flux and length/diameter (L/D) ratio, also known as the effective collimation ratio (Kobayashi, 2001). However, these two quantities do not provide a full characterization of the beam, nor do they allow for an accurate comparison between radiography facilities (Kobayashi, 2001). The image quality possible at a given facility is highly dependent on the geometry of the facility's beamline and the energy spectrum of the corresponding beam (ASTM International, 2002). Full characterization of a neutron beam enables an easy comparison between radiography facilities.

This project describes the characterization of the radiography facility at the Idaho National Laboratory's Neutron Radiography reactor (NRAD). The NRAD beamline has not been characterized since the reactor core's highly enriched uranium fuel was replaced with low enriched uranium fuel. The indicators developed in this project will improve upon the beam quality indicators already in place at the NRAD and full characterization of the beamline will benefit future radiography work at the facility.

The energy spectrum of a beamline is highly dependent on the neutron source for that beamline (Kobayashi, 2001). While a nuclear reactor is the most common neutron source for neutron radiography, several other neutron sources may be used, including sub-critical assemblies, pulsed neutron generators, and radioactive neutron sources (ASTM International, 2002). The experiments described in this work provide a complete characterization of the NRAD beamline and are designed to be easily tailored to different radiography systems and different neutron sources. The characterization experiments provide baseline measurements which can be used to track changes in the facility. 
Through this project, the NRAD now has access to the most recent ASTM beam quality indicators as well as an ASTM specified device for measuring the effective collimation ratio. Analysis of radiographs of these indicators provides meaningful information on image sharpness and resolution. The experimental results also serve to validate an existing simulation of the radiography system, which provides an important radiographic analysis tool for the facility.

The capability to accurately simulate an expected radiograph would be useful to the NRAD facility and would allow the measurement of properties such as density or thickness by comparing simulations with actual radiographs. Modeling the film response to exposure from an activated metal foil is necessary in order to accurately simulate the radiographic process. A new film characteristic curve developed for this project relates foil activity to film optical density and provides the necessary data for a film response model. The film response model creates an image simulation based on data from the existing MCNP model of the neutron beamline.

The film response simulation coupled with the MCNP beamline model expands the simulation capability of the NRAD. Better characterization of the radiography system allows for better image simulation. The more accurate the simulation capabilities of the NRAD are, the more useful the information each radiograph can provide.

The project has five related objectives:

- Measure the effective collimation ratio, beam quality, and divergence for the east radiography beamline at the $\mathrm{NRAD}$,

- determine the neutron energy spectrum for the beam using a foil activation and model reference technique,

- quantify the neutron beam profile for the facility,

- develop a characteristic curve relating the optical density of an NRAD radiograph to activity, and 
- use the new characteristic curve as part of a simulation methodology to accurately predict radiographic images at the NRAD.

The effective collimation ratio, beam quality, and beam divergence should be measured regularly at any neutron radiography facility. Objective 1 provides a baseline measurement of these quantities to aid the facility in tracking radiographic quality over time.

The energy spectrum of a neutron beam generally remains constant over time; however, changes to the core configuration, fuel, or core materials can change the energy spectrum significantly. Knowledge of the energy spectrum is necessary for the facility to quantitatively compare radiographs. Objective 2 validates the current neutron energy spectrum used at the NRAD.

The shape of the neutron flux profile affects the useable area of a neutron beam, and quantifying the profile assists in interpreting radiography results from that beam. The facility can make image adjustments based on the measured flux profile to improve the quality of the resulting neutron radiographs. Objective 3 measures the flux profile and provides the NRAD with a way to do so again in the future.

Simulating film-based radiographs requires the ability to model the film's exposure. Transfer method radiography primarily exposes film to beta particles. Objective 4 generates a characteristic curve based on beta particle and gamma ray exposure to the radiographic film used at the NRAD as input to the image simulations.

A simulation must be validated in order to be useful. Objective 5 validates the image simulation program by comparing the optical densities of a real radiograph of a polyethylene step block to a simulated image of the step block.

The suite of experiments described in this report can be easily tailored to the characterization of any neutron radiography facility. The simulation code can be edited to model any metal foil activation or film exposure. The experiments which produce the data for the film characteristic curve are easy to duplicate for any film-foil combination. 
Chapter 2 explains the concepts necessary to understand the characterization experiments and the image simulation. It also explains how these experiments benefit a neutron radiography facility as well as describing the experimental procedures and results. Chapter 3 describes the results of the characterization experiments. Chapter 4 describes the results of the experiments needed to determine the characteristic curve and the image simulation validation. Chapter 5 summarizes the results and makes final conclusions regarding the NRAD neutron radiography facility. Chapter 6 provides recommendations for future research. 


\section{CHAPTER 2}

\section{BACKGROUND}

This section describes the basic concepts related to neutron radiography, the Neutron RADiography reactor (NRAD), and the fundamentals of each characterization experiment. This section also explains radiographic film exposure, development, and scanning.

\subsection{Neutron Radiography}

Neutron imaging is a complementary technique to $X$-ray imaging (Berger and Iddings, 1998). While $X$-rays pass through light material and are attenuated by dense materials like metals, neutrons are attenuated by materials containing hydrogen and boron, and pass easily though most metals (Berger and Iddings, 1998).

A neutron radiograph is formed when an object is placed in a neutron beam in front of an image plane (see Figure 2.1) (Berger and Iddings, 1998). Neutrons from the neutron source are attenuated by the object being imaged and produce an image at the image plane as a representation of the neutron flux at that point (Nemec et al., 1995).

The image plane, also called the detector, is a combination of a material which interacts with neutrons to produce light or electrons and a material which records the emitted radiation as an image (Heller and Brenizer, 2010). These materials can include gadolinium-doped screens, dysprosium or indium foils, and neutron sensitive micro-channel plates (Crow, 2010; Craft and

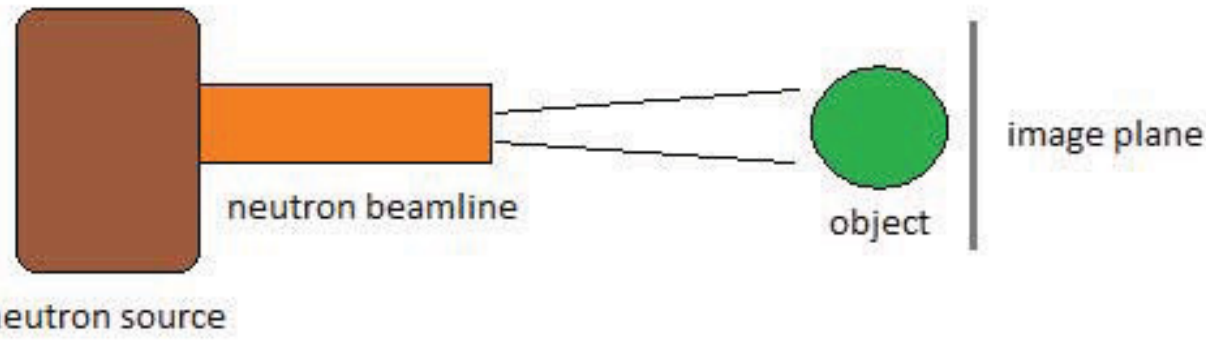

Figure 2.1. Neutron radiography block diagram. 
King, 2011). The image plane can produce either a digital or analog (film) image, depending on the type of conversion. A micro-channel plate contains many small, neutron-sensitive, channels which can provide a direct readout of the neutron image (Crow, 2010). A conversion foil and film provide an image after irradiation of the foil, mating the foil with the film, and developing the film. A more complete discussion of radiographic conversion can be found in Section 2.1.2.

The neutron source shown in Figure 2.1 can be a nuclear reactor, accelerator, or radioisotope source (Arai and Crawford, 2010). All of these source types have been used to perform neutron radiography, but accelerators and reactors provide the highest neutron fluxes and highest quality images. Radioisotope sources are generally more portable than reactors or accelerators (Arai and Crawford, 2010).

The neutron beamline (Figure 2.1) is generally a simple tube of concrete or metal which isolates neutrons in a certain solid angle (Heller and Brenizer, 2010). Since neutrons have no charge, they cannot be focused like electrons or protons, and will diverge upon exiting the beam tube (Heller and Brenizer, 2010).

The following section describes the Neutron RADiography (NRAD) reactor facility.

\subsubsection{Neutron Radiography Reactor}

The NRAD is a Mark II, $250 \mathrm{~kW}$ Testing, Research, Isotopes General Atomic (TRIGA)Fuel Life Improvement Program (FLIP) conversion reactor (Stephens, 1978). The neutron beamline exits the core on the east side of the reactor through an aperture and collimator and enters the imaging station (see Figure 2.2) (Stephens, 1978). The aperture consists of a combat grade boron nitride sheet with a circular opening (Figure 2.3). The sheet can be raised and lowered to allow for three different aperture sizes (corresponding to Length to Diameter (L/D) ratios of 50,125 , and 300) (Stephens, 1978). The L/D of 125 is the most commonly used setting at the NRAD. The collimator is a simple tube made of boral lined concrete with an inner 


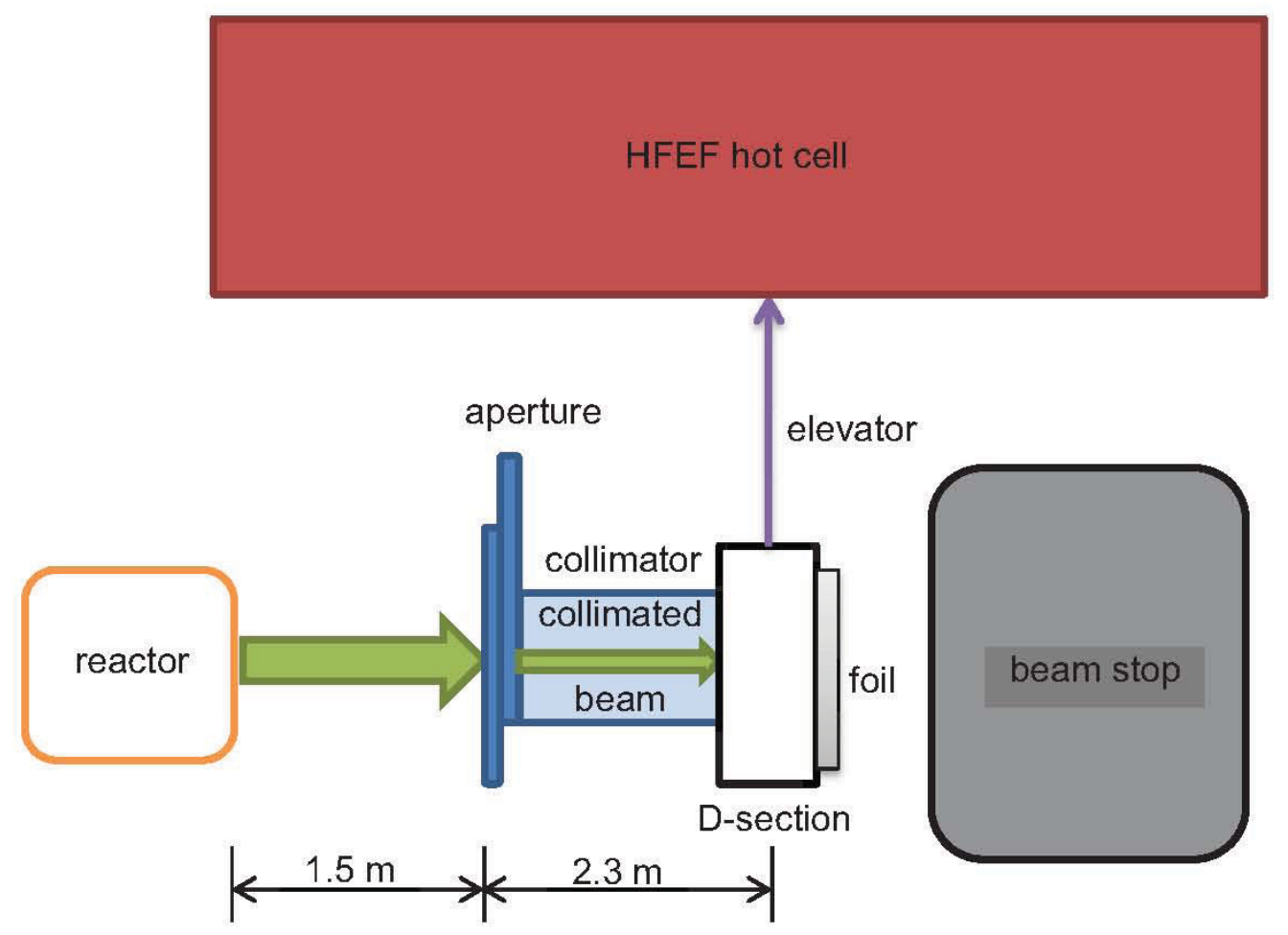

Figure 2.2. Schematic of the NRAD neutron radiography facility.

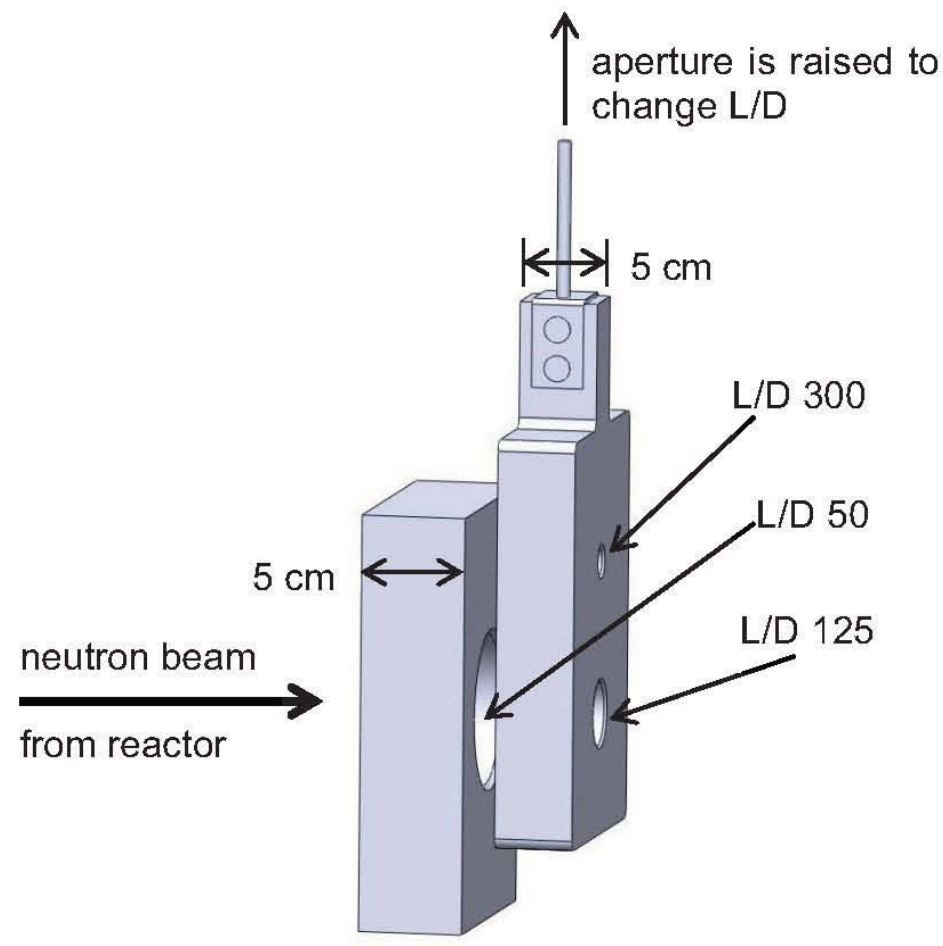

Figure 2.3. NRAD beamline aperture mechanism. 
diameter of 18 inches (Stephens, 1978).

The NRAD is located below the main hot cell in the Hot Fuel Examination Facility (HFEF) (see Figure 2.2). This location allows for the radiography of fuel elements and other highly radioactive material lowered from within the cell. An elevator (Figures 2.2 and 2.4) positions samples in the neutron beam and raises them back into the cell when the neutron exposure is complete. The cross section of the elevator tube at the point that it intersects the neutron beam is D-shaped and is known as the "D-section" (see Figure 2.4). This allows the foil cassette to be pressed against the elevator tube, as close as possible to the object being imaged (Stephens, 1978). The platen presses the cassette against the flat part of the D-section and holds it in place during radiography. The transfer method of radiographic conversion, explained in Section 2.1.2, produces the radiographs at the NRAD.

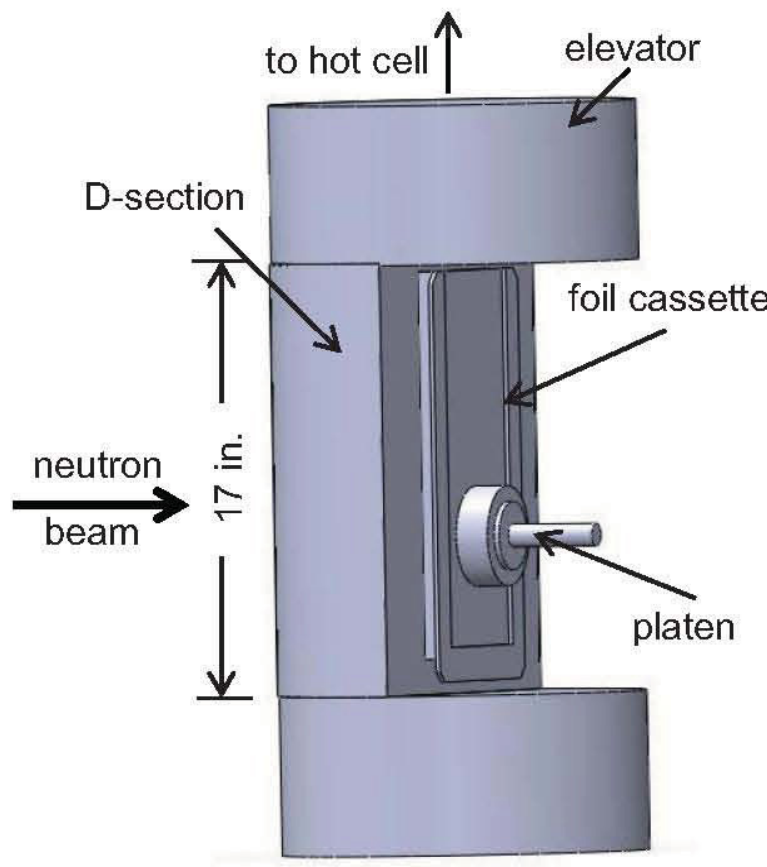

(a) D-section of sample elevator.

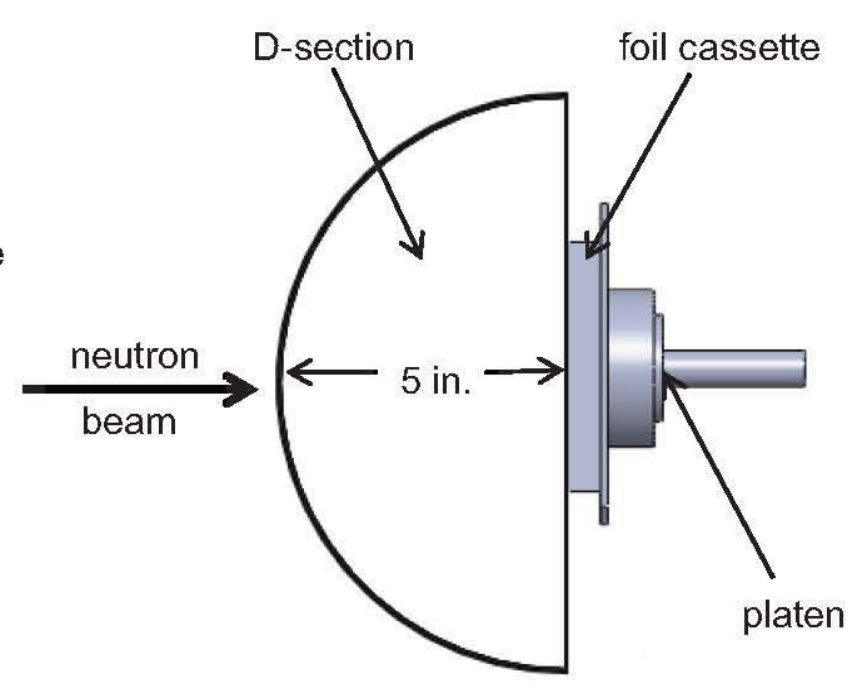

(b) Top-down view of D-section.

Figure 2.4. D-section of the NRAD sample elevator showing foil cassette and platen. 


\subsubsection{Neutron Radiography Conversion Processes}

In neutron radiography, conversion refers to the method of generating the radiographic image (Heller and Brenizer, 2010) and can be direct or indirect (Heller and Brenizer, 2010). Direct conversion utilizes a scintillation material which immediately produces light or other radiation each time a neutron interacts with it (Heller and Brenizer, 2010). The light from these interactions exposes a film, placed in contact with the scintillator (Heller and Brenizer, 2010). In direct digital conversion, a micro-channel plate reads neutron interactions and produces an image in real time (Heller and Brenizer, 2010).

Indirect conversion, also known as the transfer method, uses a foil of material such as dysprosium or indium, which interacts with neutrons through a neutron absorption reaction (Nemec et al., 1995). Exposing the foil to the neutron beam activates the foil. After the foil has been activated, film is placed in contact with the foil in a light tight container. The beta particles and gamma rays from the decay of the activated foil expose the film. The rate of activation is proportional to the neutron flux, and thus the film exposure from the decay radiation is proportional to the amount of attenuation produced by the object being imaged, producing a radiograph of the object (Heller and Brenizer, 2010).

The direct method is typically faster than the indirect method, making it possible to obtain real time images (Crow, 2010). One advantage of the indirect method is that foil activation is insensitive to gamma radiation in the neutron beam (Heller and Brenizer, 2010). While a beam with high gamma flux may fog a direct conversion image, it will have very little effect on an indirect conversion image (Heller and Brenizer, 2010). For this reason, indirect method radiography can image radioactive materials. The indirect method can result in resolutions on par with the direct technique (Heller and Brenizer, 2010).

Section 2.1.3 explains how radiography film is developed and analyzed. 


\subsubsection{Film Processing and Optical Density}

Kodak Industrex T-200 film and AGFA Structurix D3 s.c. film are the films of choice at the NRAD and can be developed using the standard Kodak developers and fixers (Quinn and Sigl, 1980). The T-200 film images the indium foils, and the D3 s.c. film images the dysprosium foils. A radiography darkroom should be humidity controlled to between $40 \%$ and $60 \%$ relative humidity to minimize static discharge marks on the film (GE Sensing and Technology, 2006; Quinn and Sigl, 1980). The developer and fixer chemicals are temperature sensitive, so the room must be also be kept between $60^{\circ} \mathrm{F}$ and $70^{\circ} \mathrm{F}$ (GE Sensing and Technology, 2006; Quinn and Sigl, 1980). Any variation in temperature produces a variation in film development time and can result in over or under developed film (Quinn and Sigl, 1980). The film needs to be in contact with the foil for the length of the exposure. At the NRAD, a vacuum sealed cassette keeps the film and foil in contact without moving. After the exposure is complete, the film can be developed by following the development procedures recommended by Kodak (Quinn and Sigl, 1980). All film handling and storage should occur within the dark room. Unexposed film should be stored individually in a horizontal position, and should not be in contact with other films.

Optical density is the amount of light transmitted through a developed film (McNaught and Wilkinson, 2006). A high optical density corresponds to a film exposed to a significant amount of radiation. A low optical density corresponds to a film exposed to less radiation. Typical optical densities range from 1.0 to 3.5 (Gonzalez-Lopez, 2007), which covers film shades from very light grey (1.0) to almost black (3.5). In neutron radiography, high optical density regions correspond to high neutron fluence regions, and vice versa.

The film density is dependent on the properties of each specific film and each film has a characteristic curve which shows the exposure necessary for the film to reach a certain optical density (Quinn and Sigl, 1980). A characteristic curve plots a film's optical density as a function of the log of the relative exposure. Figure 2.5 presents the characteristic curve for the X-ray 
response of the AGFA D3 s.c. film used at the NRAD. Figure 2.6 presents the characteristic curve for Kodak Industrex T-200 film used at the NRAD.

An unexposed film, when developed, will have an optical density greater than zero as a result of the spontaneous development of a small portion of the silver halide crystals in the film (Raj and Venkataraman, 2004). This minimum optical density is known as the fog density. Any exposure level lower than the fog density cut-off will be indistinguishable from an unexposed film. For most industrial X-ray film, the fog density is between 0.1 and 0.2 optical density (Raj and Venkataraman, 2004).

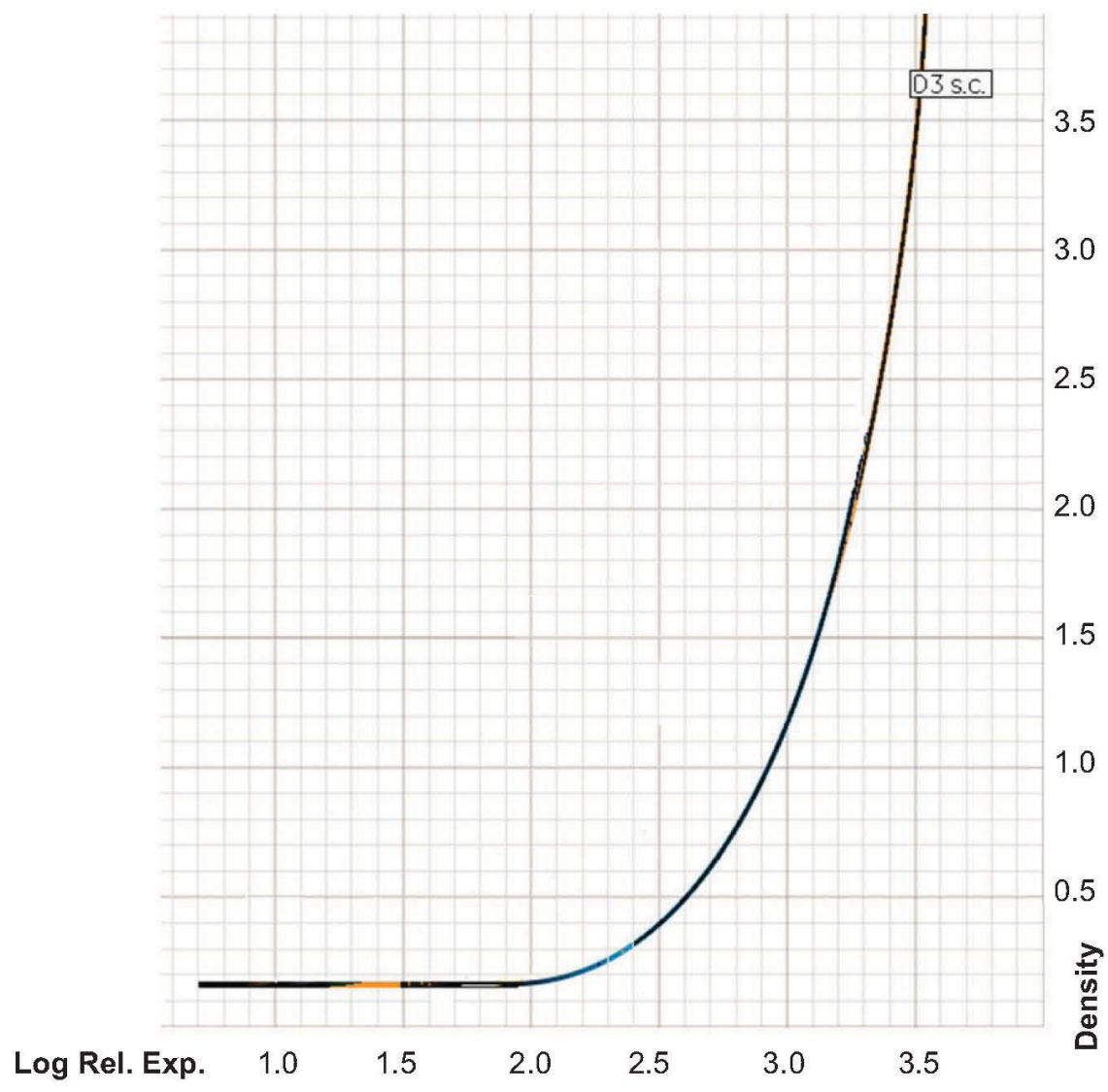

Figure 2.5. Characteristic curve for AGFA D3 s.c. X-ray film (GE Sensing and Inspection Technologies, 2008). 


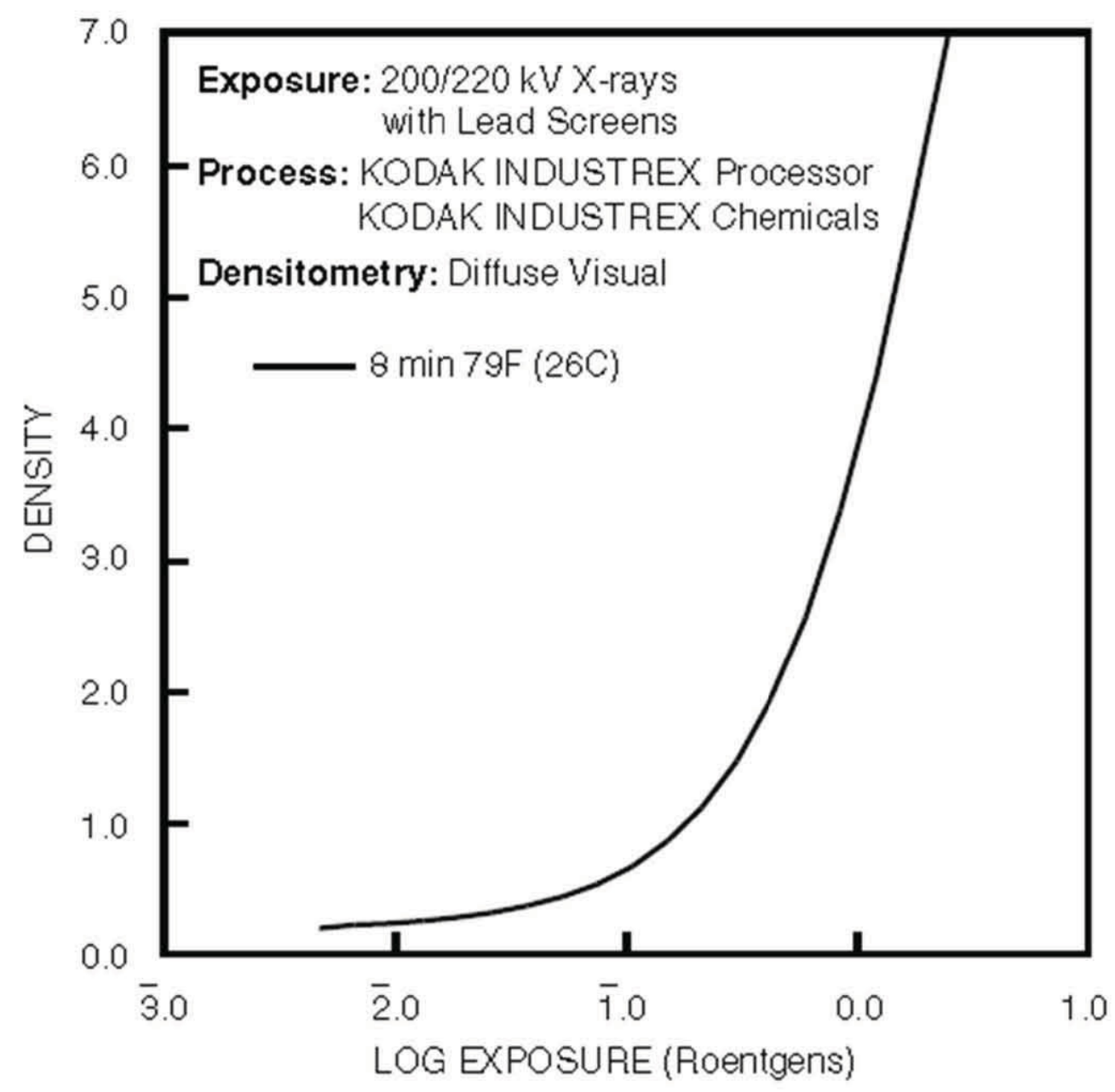

Figure 2.6. Characteristic curve for Kodak Industrex T-200 X-ray film (Carestream, 2010).

Proper exposure is usually a trial and error process, as most of the characteristic curves are plotted against the relative exposure. An initial exposure, which is analyzed by a densitometer, is needed to determine how much longer or shorter the exposure must be to achieve the desired film density (Quinn and Sigl, 1980). When using the indirect method, the highly activated portions of the dysprosium or indium foil cause the corresponding parts of the film to darken more than the parts with lower activity. Thus, high optical density corresponds to high neutron fluence at the image plane.

Characteristic curves for the industrial $\mathrm{X}$-ray film used in commercial neutron radiography relate known exposure levels to changes in film optical density (Quinn and Sigl, 
1980). Exposure is a valid measure only for photon radiation, such as X-rays and gamma rays (Hendee and Ritenour, 2002). The metals most commonly used for the transfer method, indium and dysprosium, decay by beta emission, and radiate primarily beta particles (Pritychenko, et al., 2006). The absorbed dose from the beta radiation cannot be directly related to the units of exposure used to generate the characteristic curves (Hendee and Ritenour, 2002). A characteristic curve relating optical density to either activity or absorbed dose from an activated metal foil has not been found, and a characteristic curve relating foil activity to film optical density is needed for radiography involving metal foil activation.

A scanner properly calibrated to read optical densities digitizes film for subsequent optical density analysis (Döler et al., 1994). Scanning a calibrated step wedge with known optical densities and comparing each region of known optical density to the average pixel values produced by the scanner generates a curve of optical density versus pixel value (Döler, et al., 1994).

The following section details the important parameters of neutron beams relevant to neutron radiography.

\subsection{Neutron Beam Parameters}

Characterization of a neutron beam requires knowledge of all of the parameters of the beam that relate to the quality of a radiographic image. The following sub-sections explain these parameters, as well as the experiments designed to measure them as part of this project.

\subsubsection{Effective Collimation Ratio}

The effective collimation ratio of a neutron beam is also referred to as the length-todiameter (L/D) ratio (Kobayashi, 2001). A higher L/D ratio implies that the neutron beam will produce a clearer image (Kobayashi, 2001). The effective collimation ratio is different from the physical length and diameter of the beam as a result of scattering off of the beam tube, which reduces the effective collimation (Yoshii and Kobayashi, 1996). ASTM Standard E803-91 
provides guidelines for measuring the L/D of a neutron beam using a "no umbra" (NU) device (ASTM International, 1991).

The NU device consists of an aluminum u-channel with grooves cut at regularly spaced intervals (see Figure 2.7). The grooves are filled with known diameter nylon and cadmium wires. Though ASTM E803-91 specifies a wire diameter of $0.64 \mathrm{~mm}$ (ASTM International, 1991), material availability makes a $0.7 \mathrm{~mm}$ diameter wire more practical in the present application. The u-channel sits at an angle of 45 degrees with respect to the axis of the neutron beam. Radiographing the NU device with a less than perfectly collimated beam produces a penumbra in the images of the cadmium wires. A penumbra is a secondary shadow caused by neutrons striking the object from many different angles (Thornton, 2004). Figure 2.8 illustrates the relationship between the umbra and the penumbra for a NU device. Figure 2.9 illustrates conceptually how the no umbra device works. A perfectly collimated beam will produce no penumbra in the resulting images (de Almeida et al., 2005). The size of the umbra varies with distance from the film. The distance at which the umbra disappears, relative to the film, divided by the diameter of the wire, is equal to the effective ratio L/D as given by:

$$
\frac{b}{w}=L / D
$$

The NU device should be imaged no less than $25 \mathrm{~mm}$ away from the image plane. Exposure time should be long enough to produce a nominal background film optical density of 2.5 \pm 0.4 (ASTM International, 1991). The resulting image can then be analyzed to find the "no umbra" distance and corresponding L/D ratio.

\subsubsection{Beam Quality}

Beam quality is a qualitative measure of the resolution of a radiographic system (Nemec

et al., 1995). Beam quality indicators are designed to monitor consistency in the radiographic system (ASTM International, 2005). Densitometric analysis of a radiographic image of a beam quality indicator determines the thermal neutron content, scattered neutron content, gamma 


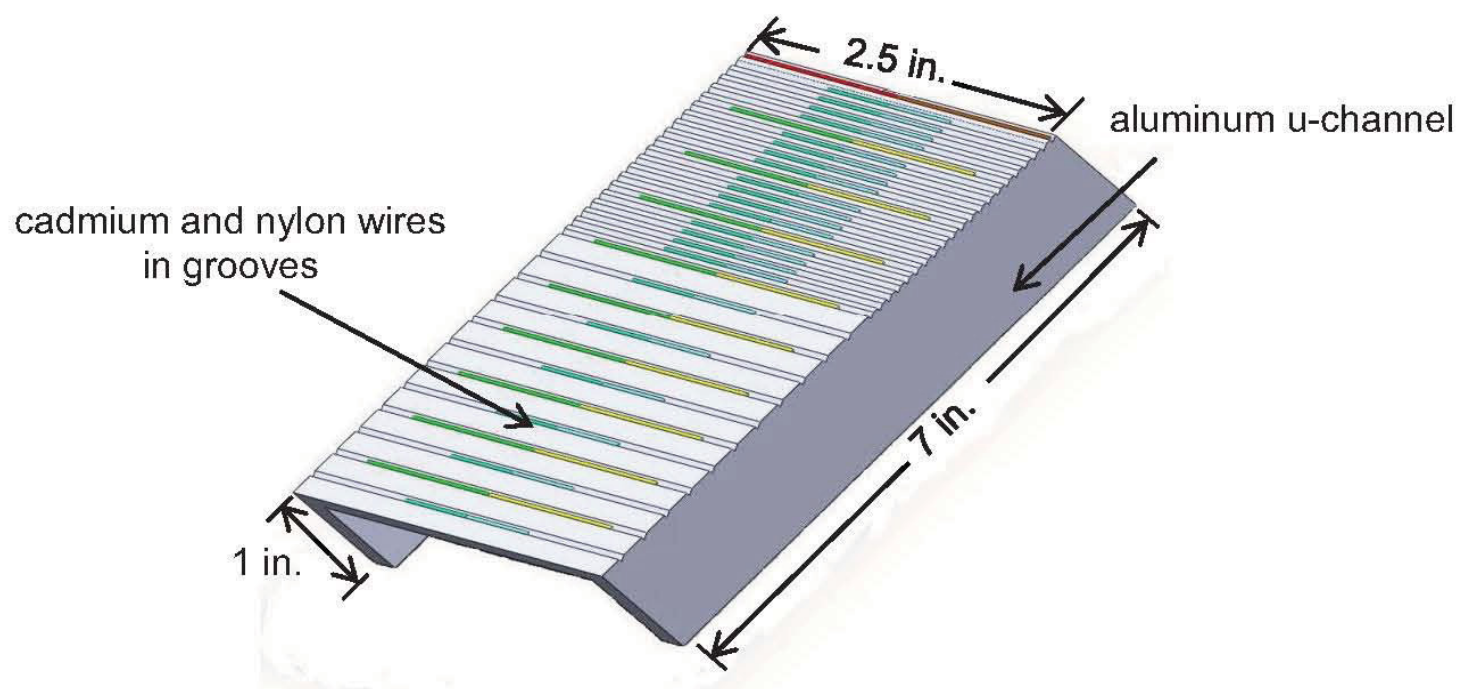

Figure 2.7. Rendered view of a no-umbra device.

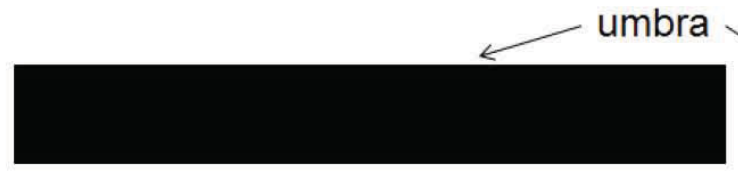

a) Perfectly collimated beam

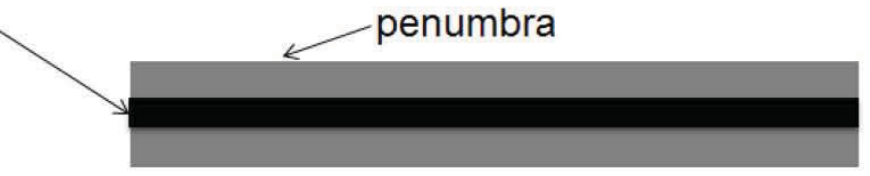

b) poorly collimated beam

Figure 2.8. Umbra and penumbra example.

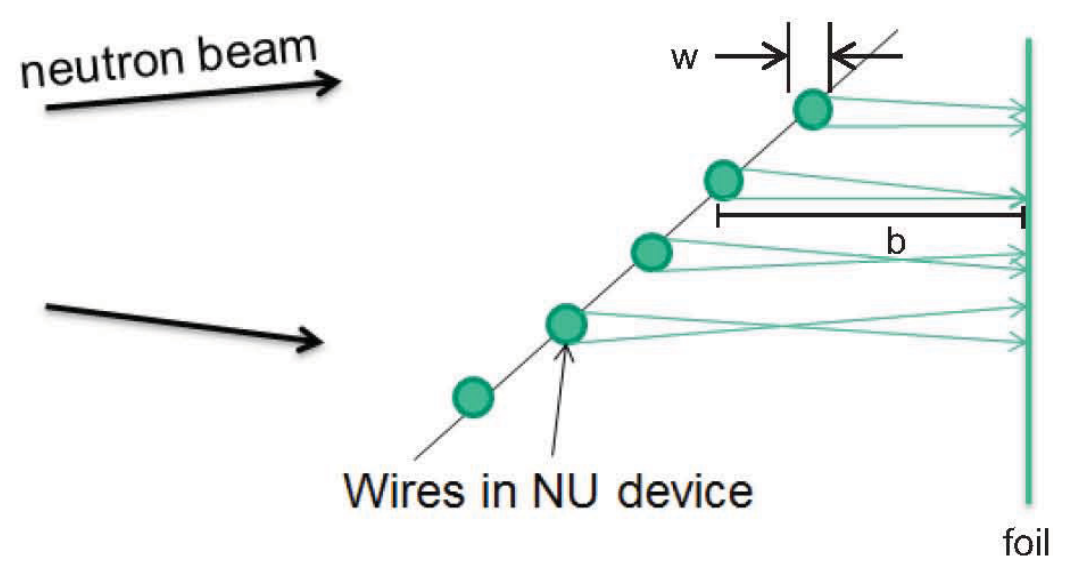

Figure 2.9. Conceptual diagram of the no-umbra device. 
content and pair production content of a neutron beam (ASTM International, 2005). These indicators are generally designed to be imaged along with each object radiographed (ASTM International, 2005).

ASTM Standard E2023-10 (ASTM International, 2010b) provides guidelines for constructing a sensitivity indicator (SI) and ASTM Standard E2003-10 (ASTM International, 2010c) provides guidelines for constructing a beam purity indicator (BPI). Figures 2.10.a, 2.10.b, and 2.11 show the details of the SI and BPI, respectively.

The sensitivity indicator is an aluminum u-channel filled with alternating strips of methylmethacrylate and aluminum (Figure 2.10.a). There are four methylmethacrylate shims under the main strips (see Figure 2.10.b). The holes in these shims are between 0.02 inches and 0.005 inches in diameter and serve as mock defects in the indicator. The top strips are milled into steps. The BPI is constructed out of a block of polytetrafluoroethylene. It contains two boron nitride dics, two lead discs and two cadmium wires (Figure 2.11). One instance of each material is set into each side of the device.

Densitometric analysis of a radiograph of each device provides information on the neutron beam (ASTM International, 2005). Since the effects of pair production and gamma content cannot be measured by the transfer method, a gadolinium foil mated with film in a light tight cassette must be used to radiograph the BPI (ASTM International, 2005). The other quantities, such as scattered neutron content, can be measured using the transfer method. The NRAD radiographed the $\mathrm{BPI}$ with dysprosium and indium foils using the transfer method.

Densitometric analysis of the beam purity indicator image produces constants for evaluating thermal neutron content (NC), scattered neutron content (S), pair production content (P), and gamma content ( $\mathrm{Y}$ ) of the beam (ASTM International, 2005). 


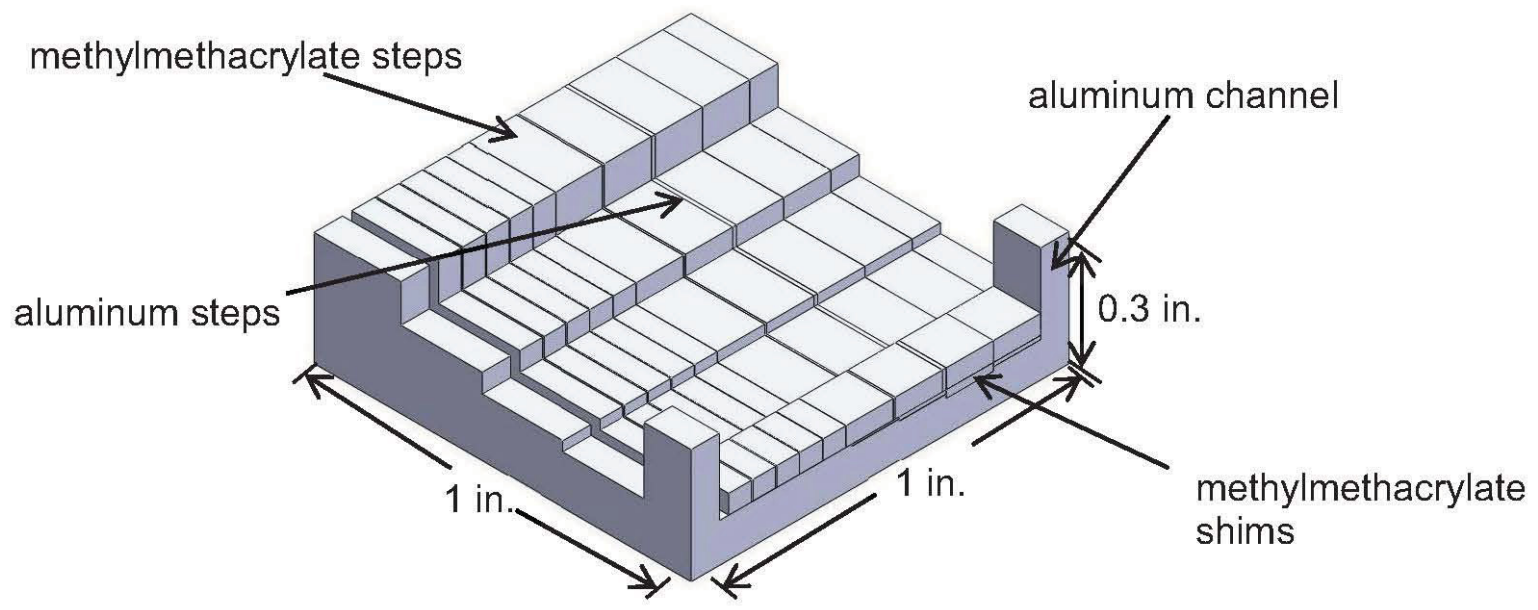

(a) Rendered view

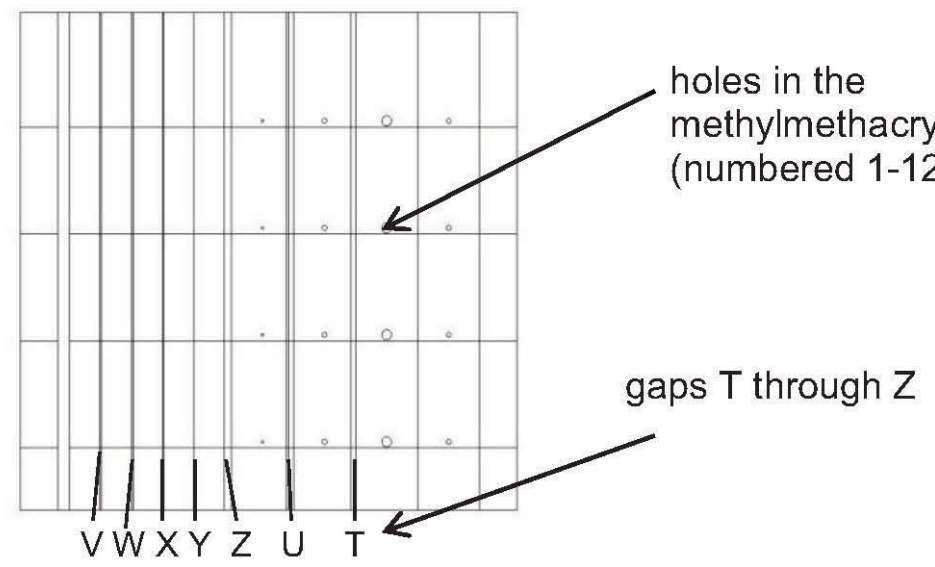

(b) Top-down view

Figure 2.10. Views of the neutron radiography sensitivity indicator.

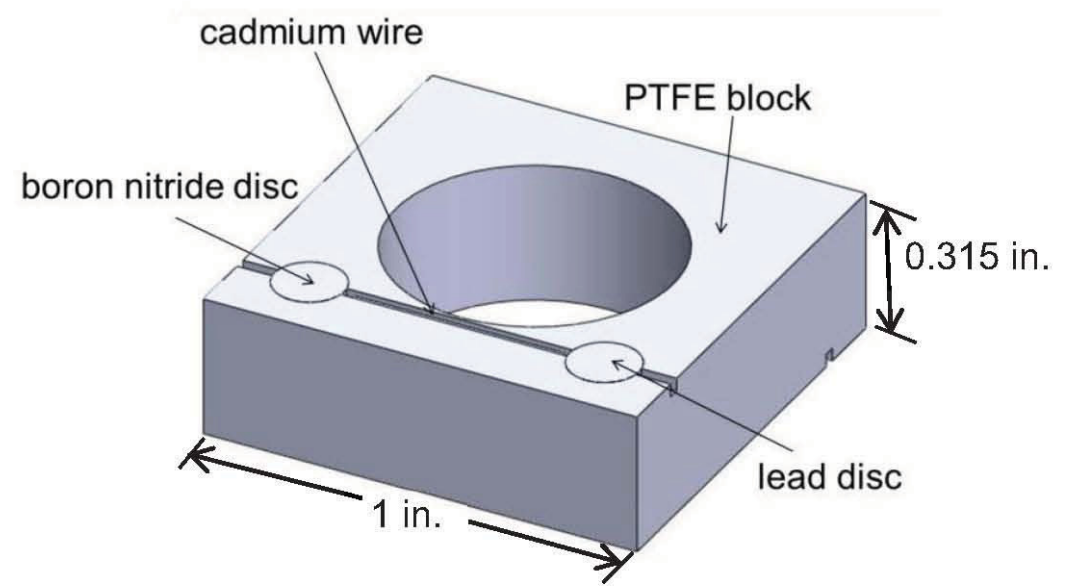

Figure 2.11. Rendering of the neutron radiography beam purity indicator. 
The equations relating the optical density in each part of the image to NC, S, P and $\mathrm{Y}$ are given below:

$$
\begin{gathered}
N C=\frac{D_{H}-\left(\text { higher } D_{B}+\Delta D_{L}\right)}{D_{H}} \times 100, \\
S=\frac{\Delta D_{B}}{D_{H}} \times 100, \\
P=\frac{\Delta D_{L}}{D_{H}} \times 100, \\
\gamma=\left(\left(D_{T}-\text { lower } D_{L}\right) / D_{H}\right) \times 100 .
\end{gathered}
$$

Tables 4 and 5 in ASTM Standard E545-05 (combined in Table 2.1), provide a rating system based on the smallest hole and thinnest aluminum shim visible in the image (ASTM International, 2005). The $\mathrm{H}$ and $\mathrm{G}$ values are image quality ratings based on the smallest hole and gap that are discernible in the SI radiograph (see Figure 2.10.b) (ASTM International, 2005). The combination of the NC, S, P, Y, H, and $\mathrm{G}$ values contribute to the radiographic category designation as shown in Table 2.1 (ASTM International, 2005).

To obtain good results, the beam purity indicator and the sensitivity indicator should be placed at least $25 \mathrm{~mm}$ from any film edge and as close as possible to the surface of the film (ASTM International, 2005). The optical density should not vary by more than $5 \%$ in five measurements across the film, including one in the center and four $25-30 \mathrm{~mm}$ from each corner of the film (ASTM International, 2005). The film should be exposed long enough that the

Table 2.1. Values of characterization parameters corresponding to radiographic category (ASTM International, 2005)

\begin{tabular}{ccccccc}
\hline $\begin{array}{c}\text { Radiographic } \\
\text { Category }\end{array}$ & NC & S & Y & P & H & G \\
\hline I & 65 & 9 & 3 & 3 & 6 & $6(Y)$ \\
II & 60 & 8 & 4 & 4 & 6 & $6(Y)$ \\
III & 55 & 7 & 5 & 5 & 5 & $5(X)$ \\
IV & 50 & 6 & 6 & 6 & 4 & $5(X)$ \\
V & 45 & 5 & 7 & 7 & 3 & $5(X)$ \\
\hline
\end{tabular}


background optical density is between 2.0 and 3.0 (ASTM International, 2005).

\subsubsection{Beam Divergence}

The divergence of a beam of particles describes the angle which the beam subtends after leaving the beam port (Arai and Crawford, 2010). This affects the placement of an object to be imaged, as an object imaged further from the beam port will experience a lower neutron flux than an object imaged very close to the beam port (de Almeida, 2005). Measurement of the shadow produced when a calibrated phantom is imaged can determine the divergence of the neutron beam (ASTM International, 2011). The difference between the size of the shadow created by the phantom and the actual size of the phantom gives an angle which is related to the angle of divergence by the inverse tangent (see Figure 2.12):

$$
\theta=\tan ^{-1}\left(\frac{r_{2}-r_{1}}{d}\right)
$$

The phantom should be imaged using both indium and dysprosium foils. The measurement of the diameter of the phantom in the resulting image and the actual diameter of the phantom provide the values needed to solve for the angle of divergence.

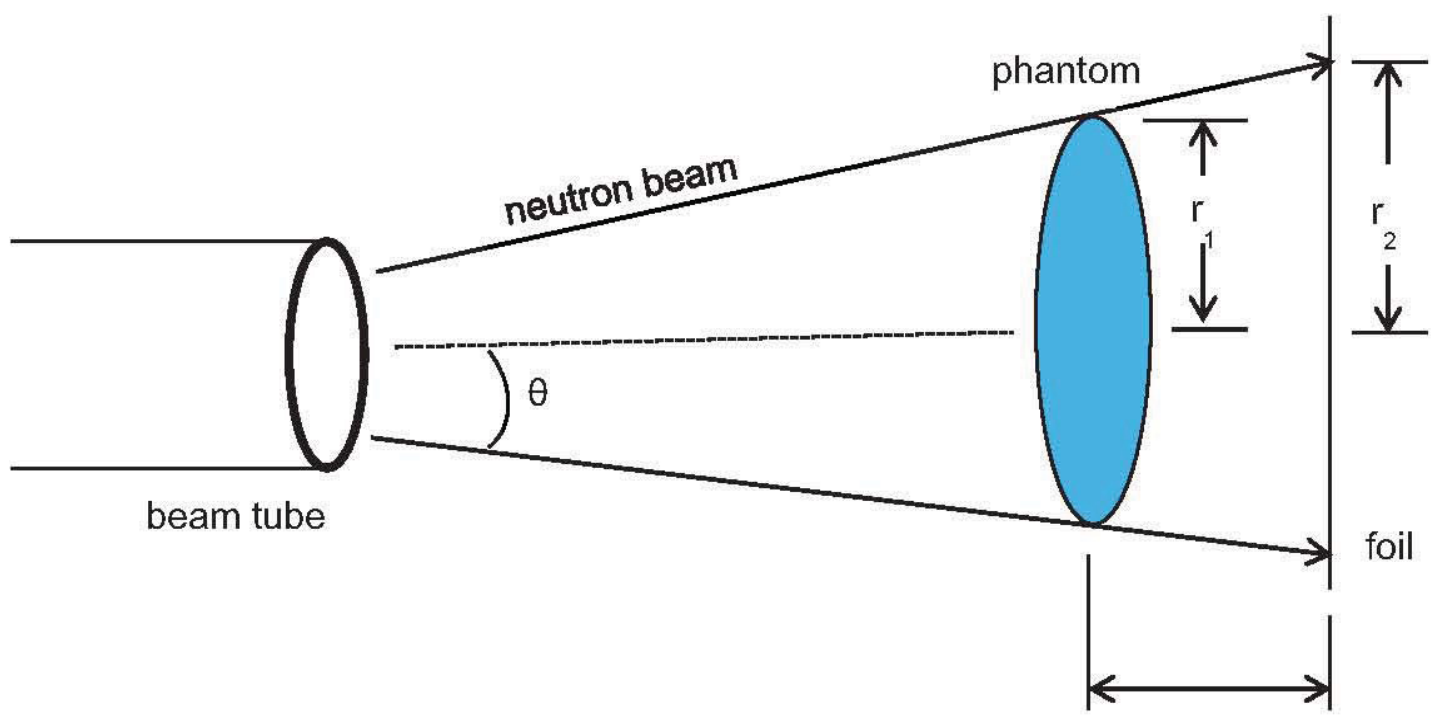

d

Figure 2.12. Beam divergence diagram. 


\subsubsection{Energy Spectrum}

Each reactor produces a slightly different neutron energy spectrum. The differences in neutron energies create different beam conditions for neutron radiography (Kobayashi, 2001). A well-known neutron energy spectrum is useful for analyzing radiographic images, as well as for comparing the images from different radiographic systems (Kobayashi, 2001).

Measurement of a neutron energy spectrum requires materials which respond in a measurable way to different neutron energies. The neutron activation technique takes advantage of the different resonance peaks in many materials' neutron absorption cross sections. For example, gold has a large, well-defined resonance peak around $4 \mathrm{eV}$, making gold a useful material for measuring the neutron flux in the $1-10 \mathrm{eV}$ range, when the foil is covered in cadmium to block thermal neutrons (Kirk and Greenwood, 1979). Since most isotope cross sections follow a $1 / \mathrm{v}$ rule in the thermal region, most foils must be covered in cadmium to measure threshold reactions in the epithermal and fast regions (Herwig, 2010). Bare foils measure reaction rates in the thermal region. Irradiating many foils with resonance peaks at energies across the spectrum produces an accurate picture of the neutron energy spectrum (Kirk and Greenwood, 1979).

Comparing calculated reaction rates for a neutron radiography beam to experimentally measured reaction rates for that beam can validate a computational model of a radiography beamline. The validated model can then provide an accurate energy spectrum in the regions with no experimental data. Traditional energy spectrum measurements use a process called unfolding to derive the energy spectrum from activation rates in the foils (Kirk and Greenwood, 1979). Unfolding programs take the foil activation rates observed in many foils and calculate the neutron energy spectrum necessary to produce the observed activations (Imel and Urbatsch, 1992). The goal of this project was to validate the energy spectrum produced by an existing Monte Carlo N-Particle (MCNP) (X-5 Monte Carlo Team, 2003) beamline model. The validation 
compared reaction rates predicted by the model to experimental reaction rates, and a full spectrum unfolding was not necessary to accomplish this task.

A recent Ph.D. thesis from Idaho State University produced a beamline model of the NRAD (Pope, 2010), with a neutron source based on a previous effort by the Idaho National Laboratory to characterize the NRAD beamline (Imel and Urbatsch, 1992). The NRAD beamline model contains all of the NRAD beamline components, foils, and cadmium covers which exist in a given experiment in the correct positions relative to the geometry of the beamline. During irradiation of the cadmium covered foils, a $0.5 \mathrm{~mm}$ cadmium cover surrounded each foil. Several foils were also irradiated bare, including tungsten, gold, indium, manganese, copper, and scandium. The MCNP model performs reaction rate calculations for each foil in the beamline. The gamma counting apparatus consists of a high purity germanium detector inside a lead cell at the Idaho National Laboratory Analytical Laboratory. ASTM standards E261-10 and E720-08 and other papers detailing the measurement of neutron beam energy spectra provide the basis for the selection and preparation of foils in this project (ASTM International, 2008; ASTM International, 2010a; Aghara et al., 2006; Auterinen et al., 2004; Howerton et al., 2006).

Table 2.2 presents the activation foils chosen for this project and Figure 2.13 illustrates a typical light water reactor spectrum (Malkawi and Ahmad, 2000) with each foil's activation energy range superimposed on the spectrum. The energy ranges covered by the foils capture the majority of the energy spectrum. The lack of foils covering the energy spectrum between 0.01 and $10 \mathrm{MeV}$ does not affect the accuracy of the characterization, as it is filled in by the MCNP model. All of the foil activation energy ranges, except the ranges for dysprosium, assume that the foils are cadmium covered. The cadmium covered foils required longer irradiation times, and therefore only four cadmium covered foils were irradiated along with the bare foils as a result of safety concerns related to handling highly activated foils. The remaining cadmium covered foils were irradiated in a separate irradiation. 
Table 2.2. Selected foils, reactions of interest, activation energy ranges, and half-lives.

\begin{tabular}{llcl}
\hline Isotope & Reaction & Energy Range $(\mathrm{eV})$ & Half-Life \\
\hline Gold-197 & $(\mathrm{n}, \mathrm{Y})$ & 3.80 to $9.20^{\mathrm{a}}$ & 2.694 days \\
Dysprosium-164 & $(\mathrm{n}, \mathrm{Y})$ & $6.00 \times 10^{-3}$ to $6.50 \times 10^{-1} \mathrm{~b}$ & 2.334 hours \\
Copper-63 & $(\mathrm{n}, \mathrm{Y})$ & $5.25 \times 10^{-1}$ to $9.60 \times 10^{3 \mathrm{c}}$ & 12.7 hours \\
Cobalt-59 & $(\mathrm{n}, \mathrm{Y})$ & $6.90 \times 10^{-1}$ to $1.43 \times 10^{2 \mathrm{c}}$ & 1925.28 days \\
Manganese-55 & $(\mathrm{n}, \mathrm{Y})$ & $4.75 \times 10^{-1}$ to $1.10 \times 10^{3 \mathrm{c}}$ & 0.10745 days \\
Scandium-45 & $(\mathrm{n}, \mathrm{Y})$ & $4.00 \times 10^{-1}$ to $4.75 \times 10^{2 \mathrm{c}}$ & 83.81 days \\
Indium-115 & $(\mathrm{n}, \mathrm{Y})$ & $9.00 \times 10^{-1}$ to $2.00^{\mathrm{b}}$ & 54.29 minutes \\
Tantalum-181 & $(\mathrm{n}, \mathrm{Y})$ & $8.90 \times 10^{-1}$ to $1.50^{\mathrm{d}}$ & 117 days \\
Tungsten-186 & $(\mathrm{n}, \mathrm{Y})$ & $5.0 \times 10^{-3}$ to $40^{\mathrm{b}}$ & 23.72 hours \\
\hline a & & &
\end{tabular}

${ }^{a}$ Aghara et al., $2006 \quad{ }^{\text {b }}$ Auterinen et al., $2004 \quad{ }^{\mathrm{c}}$ ASTM International, 2008

${ }^{\mathrm{d}}$ Howerton et al., 2006

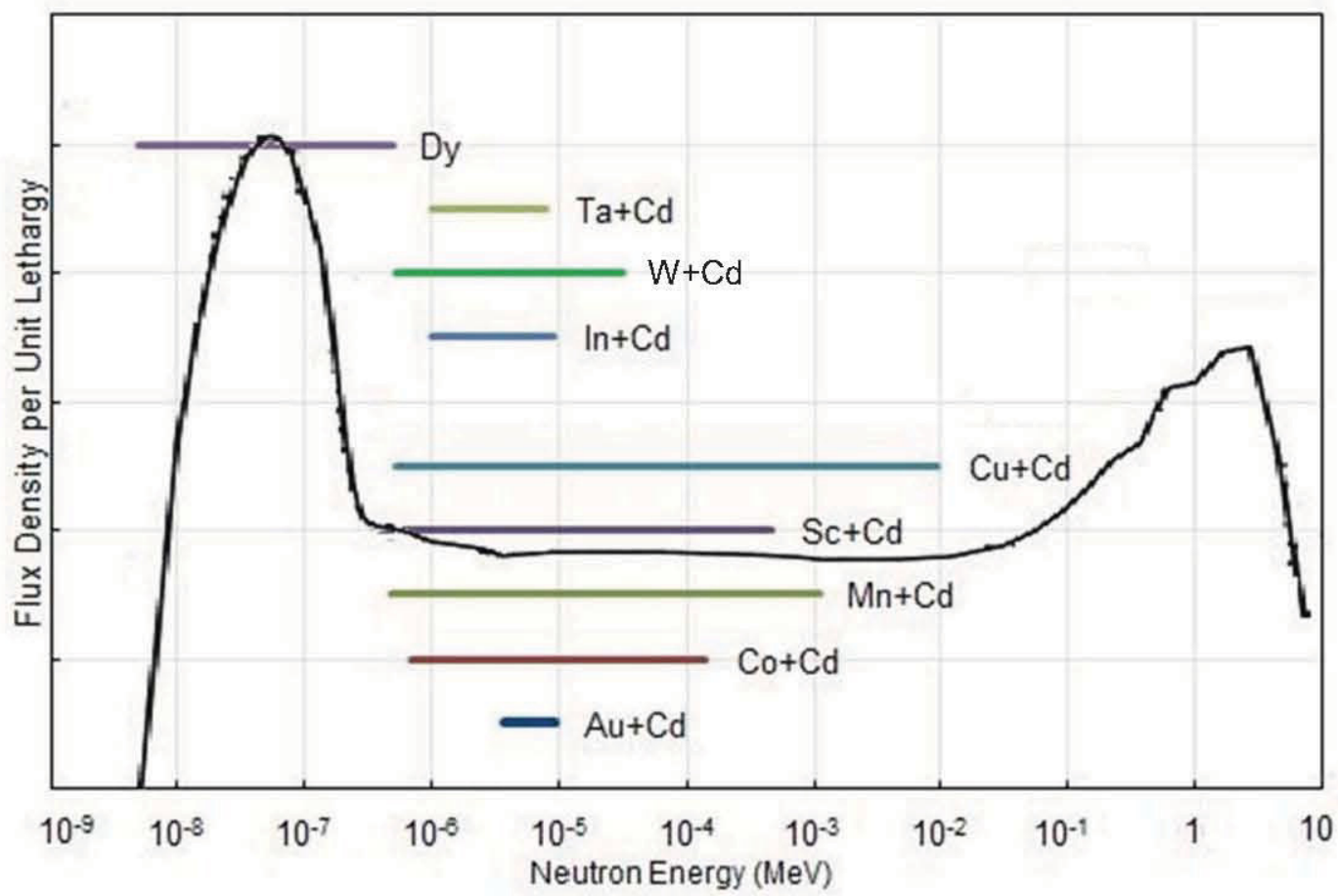

Figure 2.13. Foil activation energy ranges over-layed on a typical light water reactor spectrum taken from Malkawi and Ahmed (2000). 
Figure 2.14 shows the geometry of the MCNP model of the NRAD beamline (Pope, 2010). The neutron source in this model is a disk source located at the beamline aperture with a divergence of 5 degrees. Figure 2.15 displays the energy spectrum used in the NRAD beamline model (Imel and Urbatsch, 1992; Pope, 2010). Table 2.3 displays the energy bin numbers and bin boundaries.
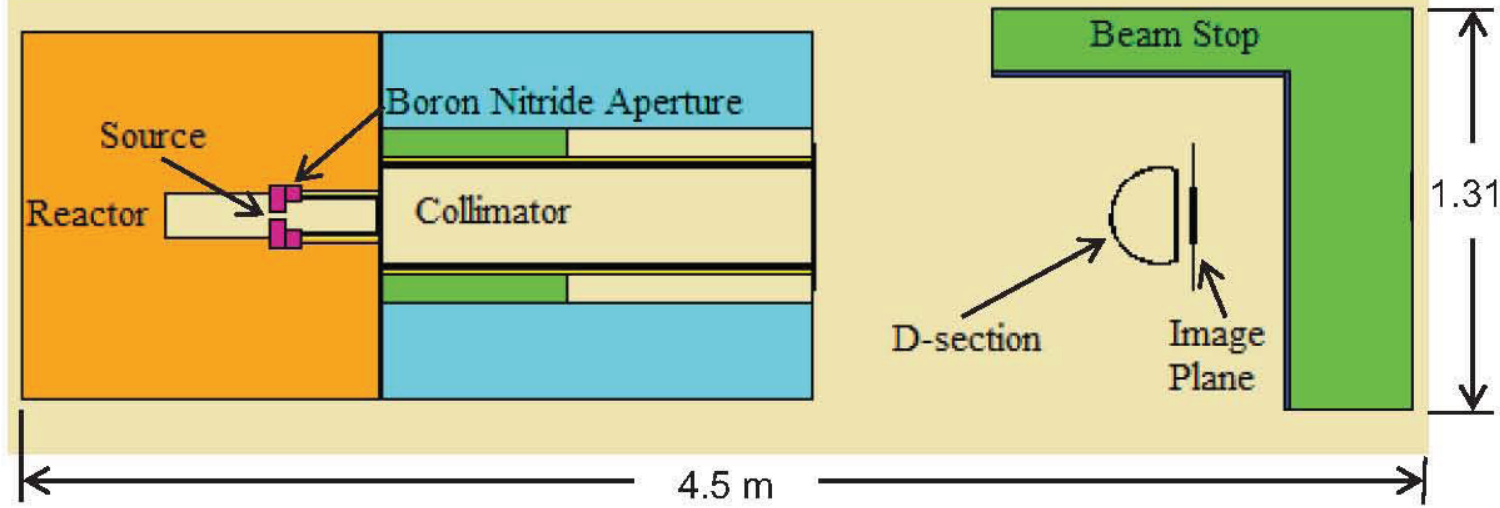

Figure 2.14. Geometry of the NRAD beamline model.

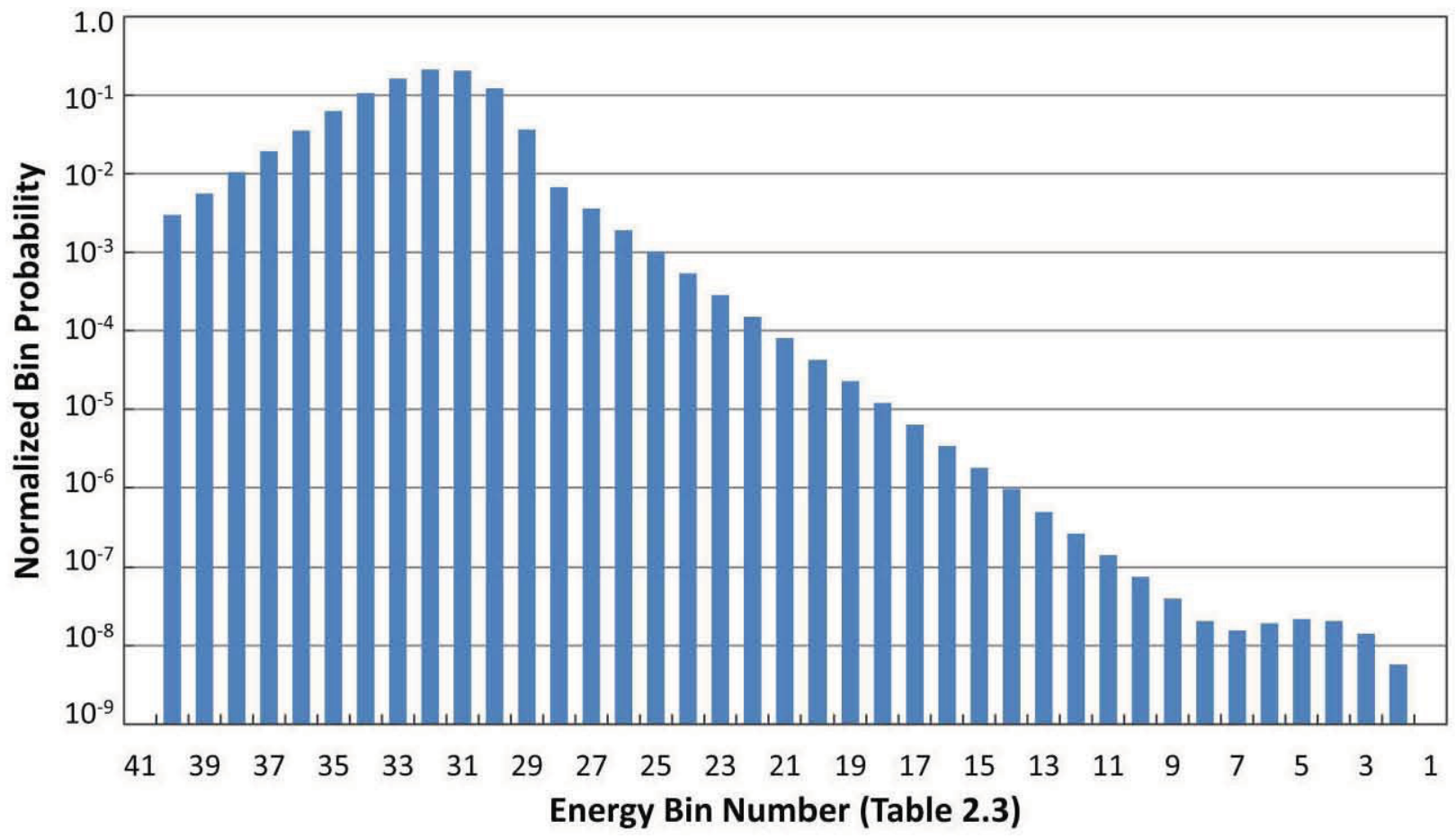

Figure 2.15. Neutron energy spectrum used in the NRAD beamline model. 
Table 2.3. NRAD MCNP model bin energies and probabilities.

\begin{tabular}{|c|c|c|c|}
\hline $\begin{array}{c}\text { Bin } \\
\text { Number }\end{array}$ & $\begin{array}{c}\text { Minimum Bin } \\
\text { Energy } \\
(\mathrm{MeV})\end{array}$ & $\begin{array}{c}\text { Maximum } \\
\text { Bin Energy } \\
(\mathrm{MeV})\end{array}$ & $\begin{array}{c}\text { Bin } \\
\text { Probability }\end{array}$ \\
\hline 41 & $1.00 \mathrm{E}-12$ & $1.00 \mathrm{E}-10$ & 0 \\
\hline 40 & $1.00 \mathrm{E}-10$ & $1.88 \mathrm{E}-10$ & $3.00 \mathrm{E}-03$ \\
\hline 39 & $1.88 E-10$ & $3.55 E-10$ & $5.62 \mathrm{E}-03$ \\
\hline 38 & $3.55 E-10$ & $6.62 \mathrm{E}-10$ & $1.05 E-02$ \\
\hline 37 & $6.62 E-10$ & 1.26E-09 & $1.94 \mathrm{E}-02$ \\
\hline 36 & 1.26E-09 & 2.37E-09 & $3.55 E-02$ \\
\hline 35 & 2.37E-09 & 4.47E-09 & $6.31 \mathrm{E}-02$ \\
\hline 34 & 4.47E-09 & 8.41E-09 & 1.07E-01 \\
\hline 33 & 8.41E-09 & $1.58 \mathrm{E}-08$ & $1.64 \mathrm{E}-01$ \\
\hline 32 & $1.58 \mathrm{E}-08$ & 2.99E-08 & 2.13E-01 \\
\hline 31 & $2.99 E-08$ & $5.62 E-08$ & 2.05E-01 \\
\hline 30 & $5.62 E-08$ & $1.06 \mathrm{E}-07$ & 1.23E-01 \\
\hline 29 & 1.06E-07 & $2.00 \mathrm{E}-07$ & $3.68 \mathrm{E}-02$ \\
\hline 28 & 2.00E-07 & 3.76E-07 & 6.77E-03 \\
\hline 27 & $3.76 \mathrm{E}-07$ & 7.08E-07 & $3.59 E-03$ \\
\hline 26 & 7.08E-07 & 1.33E-06 & $1.91 \mathrm{E}-03$ \\
\hline 25 & 1.33E-06 & 2.51E-06 & $1.01 \mathrm{E}-03$ \\
\hline 24 & $2.51 \mathrm{E}-06$ & $4.73 E-06$ & 5.37E-04 \\
\hline 23 & 4.73E-06 & 8.91E-06 & 2.85E-04 \\
\hline 22 & 8.91E-06 & $1.68 \mathrm{E}-05$ & 1.51E-04 \\
\hline 21 & 1.68E-05 & $3.16 \mathrm{E}-05$ & 8.04E-05 \\
\hline 20 & $3.16 \mathrm{E}-05$ & 5.96E-05 & 4.27E-05 \\
\hline 19 & $5.96 \mathrm{E}-05$ & $1.12 E-04$ & 2.27E-05 \\
\hline 18 & $1.12 E-04$ & $2.11 \mathrm{E}-04$ & 1.20E-05 \\
\hline 17 & $2.11 \mathrm{E}-04$ & 3.98E-04 & 6.39E-06 \\
\hline 16 & $3.98 \mathrm{E}-04$ & $7.50 \mathrm{E}-04$ & $3.39 E-06$ \\
\hline 15 & 7.50E-04 & $1.41 \mathrm{E}-03$ & 1.80E-06 \\
\hline 14 & $1.41 \mathrm{E}-03$ & 2.66E-03 & 9.56E-07 \\
\hline 13 & 2.66E-03 & $5.01 \mathrm{E}-03$ & 5.07E-07 \\
\hline 12 & $5.01 E-03$ & $9.44 \mathrm{E}-03$ & $2.69 \mathrm{E}-07$ \\
\hline 11 & $9.44 \mathrm{E}-03$ & $1.78 \mathrm{E}-02$ & 1.43E-07 \\
\hline 10 & $1.78 \mathrm{E}-02$ & 3.35E-02 & 7.59E-08 \\
\hline 9 & $3.35 \mathrm{E}-02$ & 6.31E-02 & $4.03 E-08$ \\
\hline 8 & $6.31 \mathrm{E}-02$ & $1.19 \mathrm{E}-01$ & 2.09E-08 \\
\hline 7 & 1.19E-01 & 2.24E-01 & 1.57E-08 \\
\hline 6 & 2.24E-01 & 4.22E-01 & $1.94 \mathrm{E}-08$ \\
\hline 5 & $4.22 \mathrm{E}-01$ & 7.94E-01 & $2.18 \mathrm{E}-08$ \\
\hline 4 & 7.94E-01 & $1.50 \mathrm{E}+00$ & 2.07E-08 \\
\hline 3 & $1.50 \mathrm{E}+00$ & $2.82 E+00$ & $1.43 \mathrm{E}-08$ \\
\hline 2 & $2.82 E+00$ & $5.31 \mathrm{E}+00$ & 5.81E-09 \\
\hline 1 & $5.31 \mathrm{E}+00$ & $1.00 \mathrm{E}+01$ & $9.80 \mathrm{E}-10$ \\
\hline
\end{tabular}


The spectrum in Figure 2.5 and Table 2.3 was measured before the NRAD's core was replaced with low enriched fuel. The authors of the spectrum paper imply that the fast region of the spectrum may not be well modeled (Imel and Urbatsch, 1992). The activation foils were modeled in the simulations at the same location, relative to the image plane, as the experiment. The foil irradiation simulations tracked 9 billion particles and all $2 \sigma$ tally uncertainties were under $15 \%$.

\subsubsection{Flux Profile}

The flux across a neutron beam may not be uniform and tends to taper at the edges and peak in the middle (Nemec et al., 1995). Asymmetries in the flux profile may also result from asymmetries in the neutron source and beamline. The resulting flux asymmetries, known as flux tilting, can affect the quality of the radiographic image (Nemec et al., 1995). The flux profile is measured by generating blank radiographs using dysprosium and indium foils. An image editing program analyzes the scanned radiographs to yield a plot of the pixel values, and thus the optical density as a function of position.

The flux profile generated by the NRAD beamline MCNP model can then be compared to the normalized radiograph profile. Matching the simulated profile to the radiograph profile is another validation method for the MCNP model. A validated flux profile is important for

producing accurate radiograph simulations. Chapter 3 discusses the results of the characterization experiments performed at the NRAD. 


\section{CHAPTER 3}

\section{NRAD BEAM CHARACTERIZATION RESULTS}

Characterization of the NRAD beamline began in the summer of 2011. The beam purity indicator (BPI), sensitivity indicator (SI) and no umbra (NU) device have been imaged and analyzed. Four blank dysprosium foils have been imaged, the flux profile has been calculated, and the divergence phantom has been imaged. One set of bare activation foils and two sets of cadmium covered foils have been irradiated and counted. An array of gold and dysprosium foils have been irradiated and counted. The following section presents and analyzes the results from the beam characterization experiments.

\subsection{Effective Collimation Ratio Measurement}

The elevator mechanism in the HFEF hot cell lowered the NU device for the NRAD into place in the D-section. A special carrier, designed and built as part of this project (Figure 3.1), held the NU device in the D-section at a $45^{\circ}$ angle, and guaranteed that the distance between the film and the NU device was known.

The length of the NU device was approximately 1 inch too long to fit in the D-section. Removing two of the top grooves and one of the bottom grooves reduced the size of the NU device and allowed it to fit in the $D$-section. This change slightly reduces the range of $L / D$ values that the device can measure; however, hand calculations indicated that the predicted L/D ratio of the NRAD beam should be within the range that can be measured with the modified NU device. The predicted value is based on the aperture with a L/D value of 125 . Figure 3.2 shows the original and modified NU devices.

Figure 3.3 displays the radiograph of the NU device imaged on October 15,2012 . The no-umbra point was not found by visual inspection. Figure 3.4 displays a rotated and enlarged 


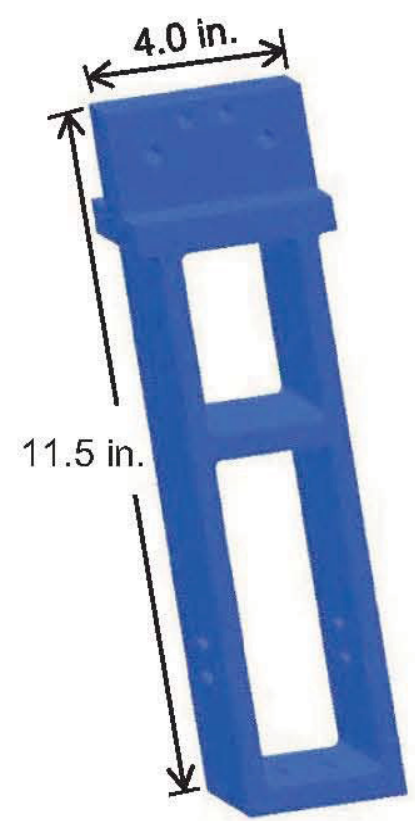

(a) No-umbra device carrier

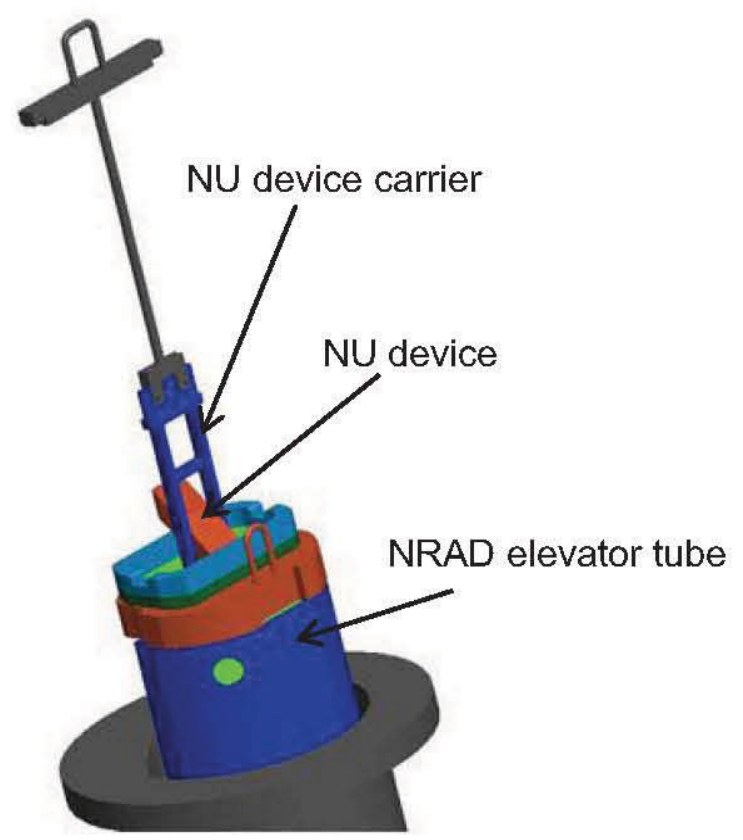

(b) No-umbra device and carrier in the NRAD elevator mechanism.

Figure 3.1. Renderings of the no-umbra device carrier.

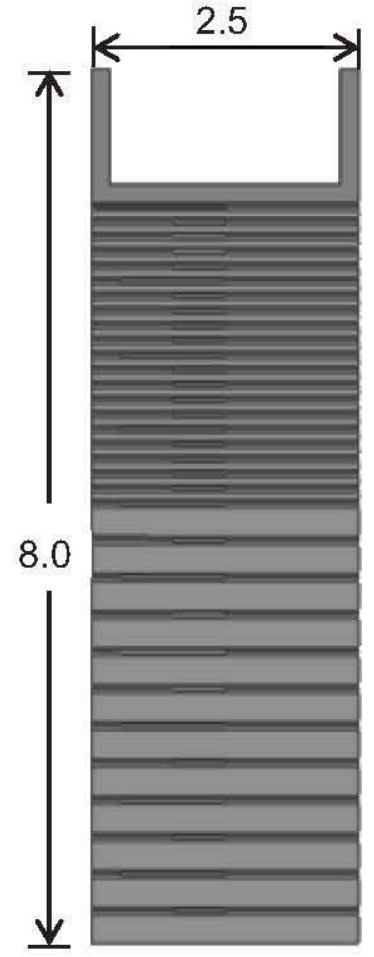

(a) Original NU device
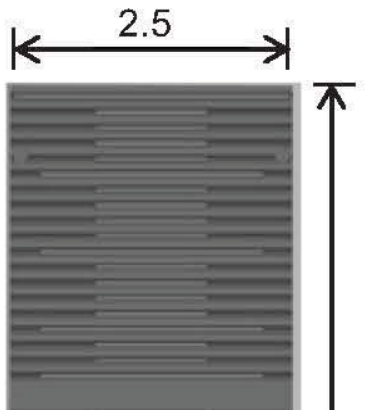

6.0

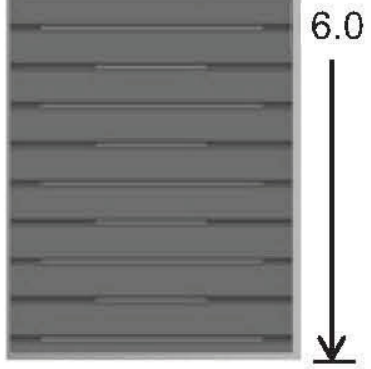

(b) Modified NU device

Figure 3.2. Renderings of the original and modified no-umbra device. 


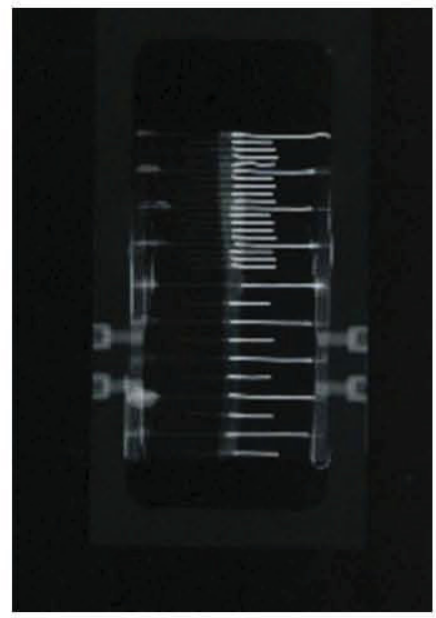

Figure 3.3. Radiograph of the NU device.

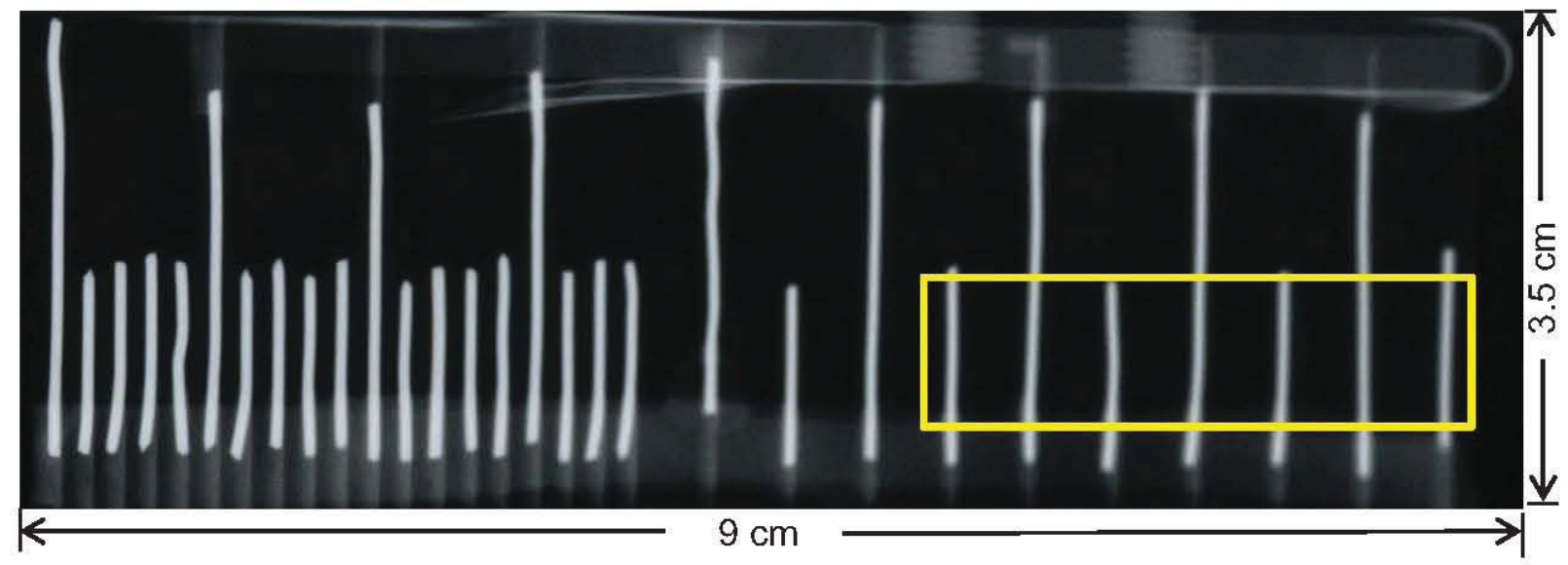

Figure 3.4. Enlarged and rotated image of the cadmium wires in the NU device.

image of the cadmium wires on the device. Figure 3.5 displays the gray value profile of the radiograph averaged along the box highlighted in Figure 3.4 .

The peaks in the profile represent the cadmium wires. The peak values are steadily decreasing, but have not reached a minimum, indicating that the no umbra point has not been reached. This indicates that the actual $L / D$ of the beam is greater than 125 , thus requiring a larger NU device or a NU device with smaller diameter wires to in order to measure the L/D. Imaging a larger device is difficult at the NRAD as a result of the constraining width of the dsection. A NU device with smaller diameter wires would increase the measureable range of L/D without increasing the size of the device. 


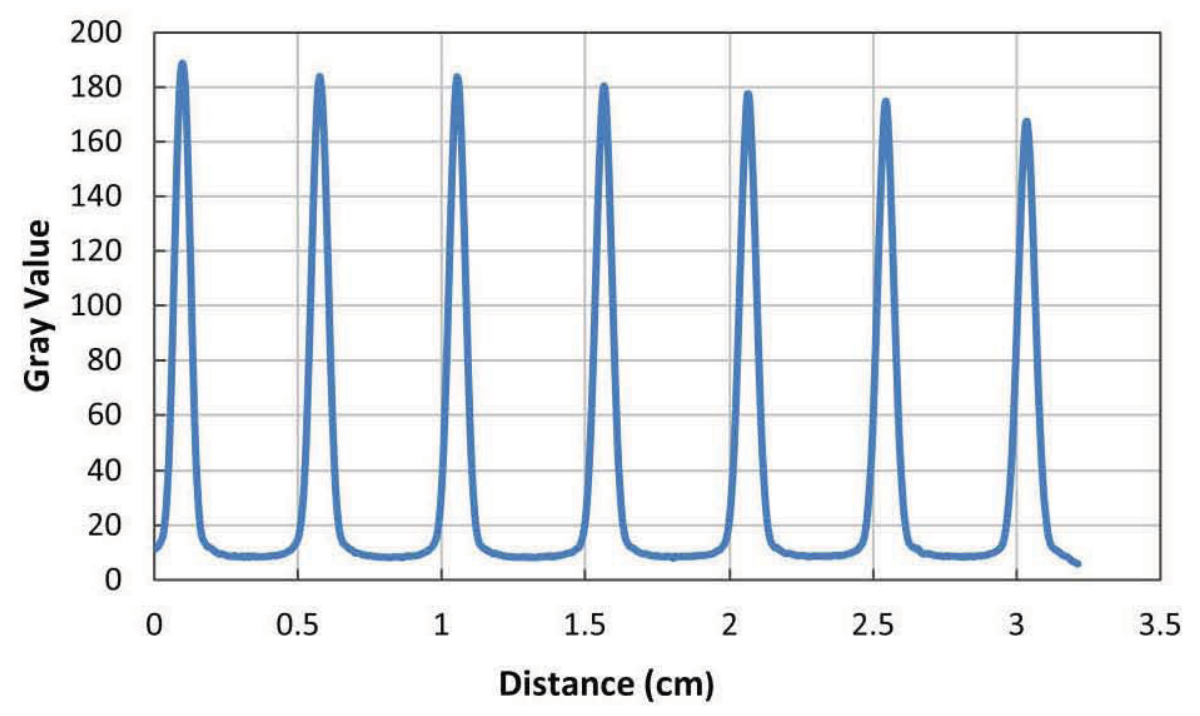

Figure 3.5. Gray value profile averaged across the box in Figure 3.4.

\subsection{Beam Quality Measurements}

The NRAD imaged the sensitivity indicator, beam purity indicator, and divergence phantom on October 18, 2012. Figure 3.6 shows the locations of the beam purity indicator (BPI), sensitivity indicator (SI), and divergence phantom mounted on the resolution test piece (RTP) carrier in the HFEF hot cell. The RTP carrier also contains an old BPI and SI. Each indicator must be enclosed in a holder in order to maneuver it in-cell, and thus the indicators are not visible in photographs of the carrier. Figure 3.7 shows the radiograph of the RTP carrier containing the $\mathrm{BPI}, \mathrm{SI}$, and divergence phantom. The RTP carrier is longer than the maximum length of a radiograph, thus the radiograph does not show all of the indicators present on the RTP.

Analyzing the image of the BPI using a densitometer produces the values listed in Figure 3.8.a for each area of the BPI. The gaps in the sensitivity indicator are labeled T through Z, with $T$ being the thickest shim $(0.010$ ") and $Z$ being the thinnest $(0.0005$ "). Shims $T$ through $Z$ correspond to $\mathrm{G}$ values of 1 through 7 respectively. The thinnest distinguishable shim in the SI 


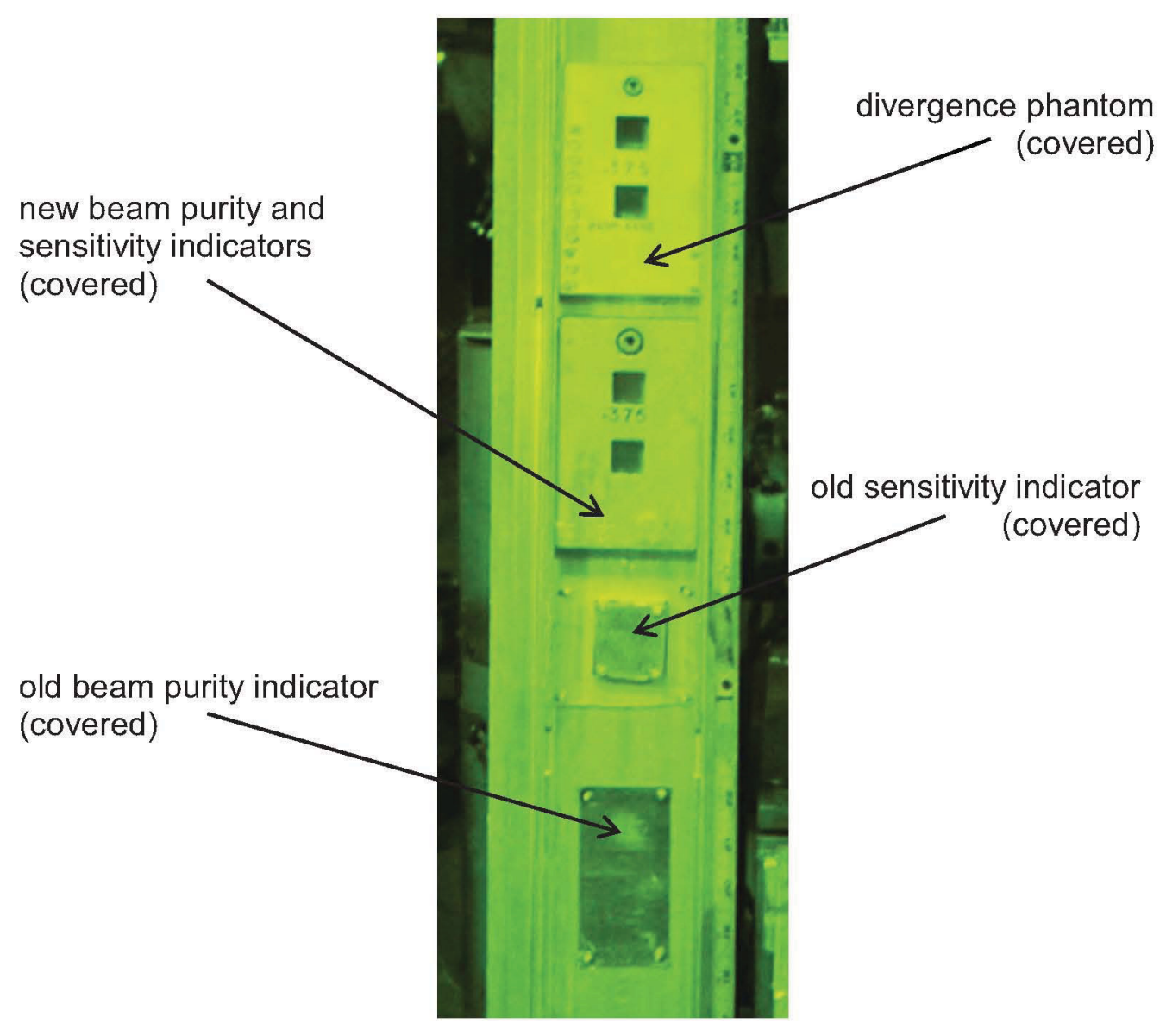

Figure 3.6. Resolution Test Piece carrier in the HFEF hot cell.

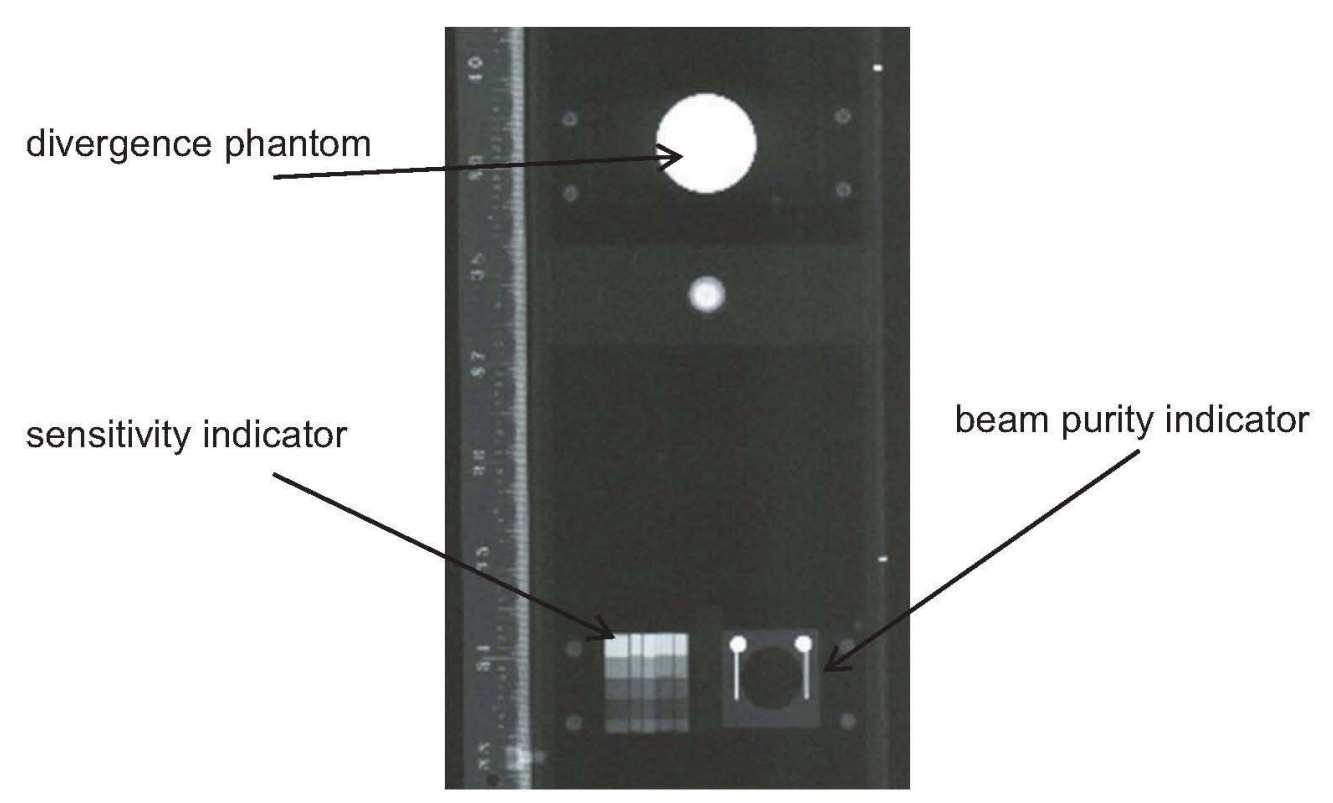

Figure 3.7. Radiograph of the beam purity and sensitivity indicators, and the divergence phantom mounted on the resolution test piece carrier. 


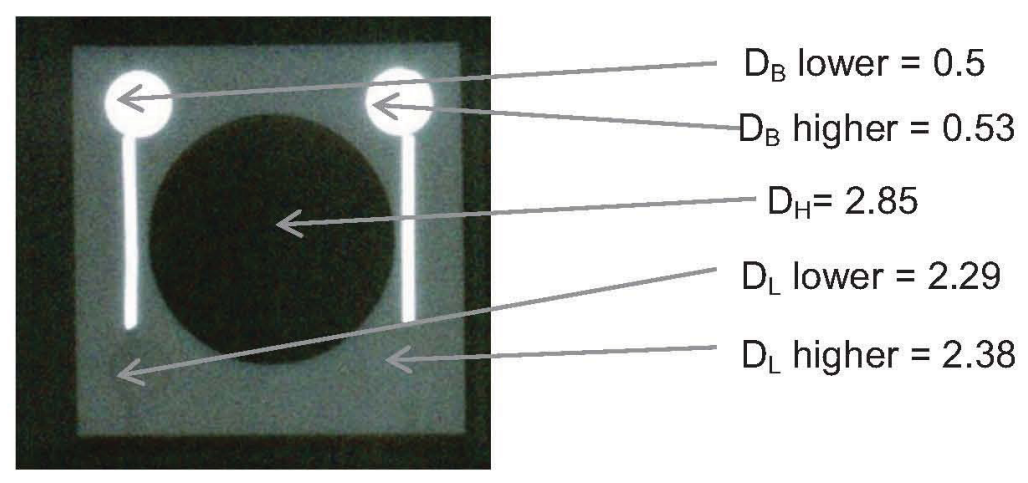

(a) Radiograph of the beam purity indicator

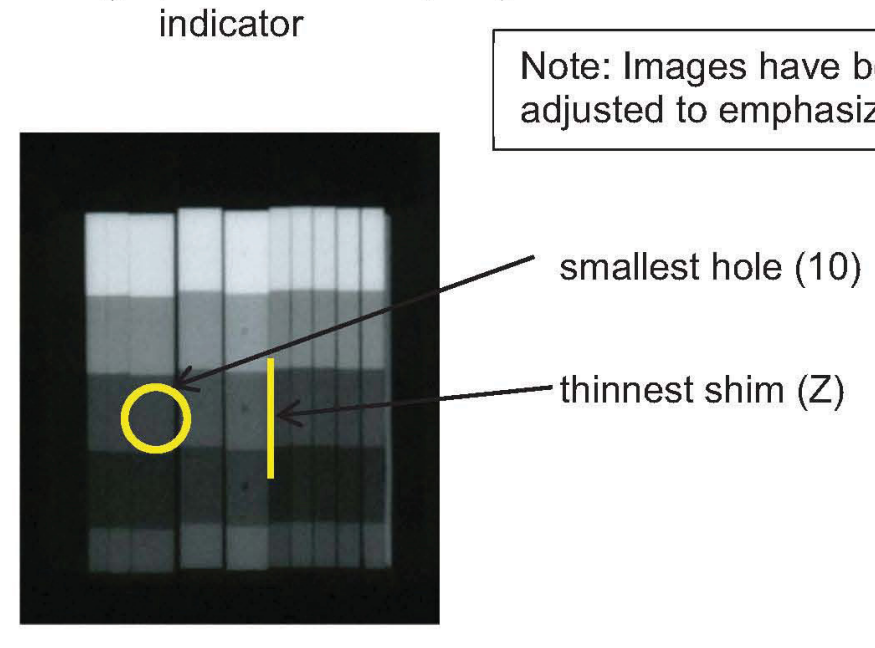

(b) Radiograph of the sensitivity indicator.

Figure 3.8. Radiographs of sensitivity and beam purity indicators.

radiograph (Figure 3.8.b) is the 0.0005 inch thick gap $Z$, highlighted in Figure 3.8, therefore, the $\mathrm{G}$ value is 7 . The smallest distinguishable hole is hole number 10 , circled in Figure 3.8.b, and thus, the $\mathrm{H}$ value is 10. Applying Equations 2.2-2.5 yielded the values listed in Table 3.1 for the beam quality.

ASTM standard E545-08 considers facilities using direct conversion radiography, and the $\mathrm{P}$ and $\mathrm{V}$ values can only be measured using direct conversion techniques (ASTM International, 2005). These values are not applicable to facilities using only the indirect transfer method. The NRAD does not have the capability to perform direct radiography using gadolinium 
Table 3.1. Radiographic category designation of the NRAD.

\begin{tabular}{ccccccc}
\hline NC & H & G & S & $\mathbf{P}$ & $\mathbf{Y}$ & $\begin{array}{c}\text { Radiographic } \\
\text { Category }\end{array}$ \\
\hline 78 & 10 & 7 & 1 & N/A & N/A & I \\
\hline
\end{tabular}

foil and measuring the $P$ and $y$ values is not possible. The remaining values are sufficient to determine the radiographic category (ASTM International, 2005). Based on the analysis of the $\mathrm{BPI}$ and SI radiographs the NRAD is a category I facility (Table 3.1).

\subsection{Divergence Measurement}

Mounting the divergence phantom on the RTP carrier created a distance of 2.4 inches between the phantom and the image plane. Figure 2.12 from Section 2.2.5 illustrates the setup of the divergence measurement. Using the standard shot time of 22 minutes and an L/D setting of 125 , the image of the phantom was 0.012 inches larger in diameter than the indicator itself. Figure 3.9 illustrates the difference in size between the divergence phantom and the image of the phantom. Using Equation 2.7, the distance from indicator to foil, and the diameter of the indicator, the divergence of the NRAD beam is $0.3 \pm 0.1$ degrees. This low angle of divergence suggests that there should not be a large variation over the image plane of the NRAD.

\subsection{Energy Spectrum}

The NRAD irradiated 21 foils for one hour on July 31, 2011, at an L/D aperture setting of 125 and a reactor power of $250 \mathrm{~kW}$. On October 16, 2012, the NRAD irradiated 17 foils for 8 hours. Table 3.2 lists the foils, their masses, and the resulting activities for both irradiations. After irradiation, the Idaho National Laboratory Analytical Laboratory measured the gamma activity for each of the product isotopes of interest.

The majority of the foils irradiated in 2011 were irradiated bare. The manganese, gold, copper and indium foils were also irradiated with $0.5 \mathrm{~mm}$ thick cadmium covers. 


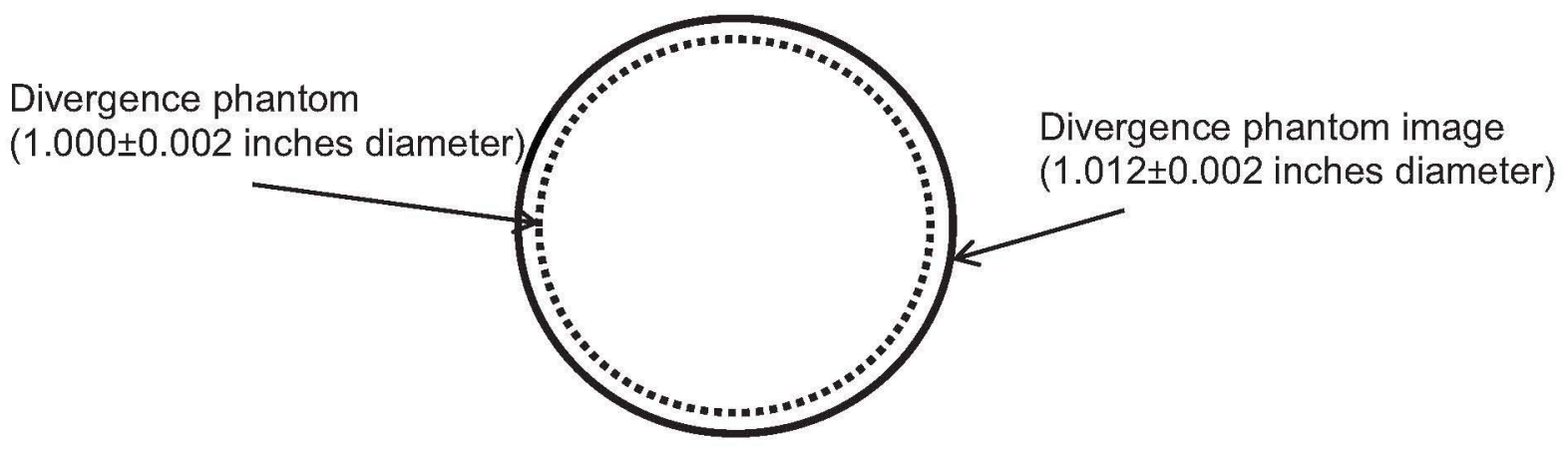

Figure 3.9. Schematic of the measured divergence phantom diameter and the actual diameter of the phantom.

The scandium foil was still radioactive from the 2011 irradiation at the time of the 2012 irradiation. The residual activity and decay time are accounted for in the resulting scandium activity. All of the foils irradiated in 2012 were covered with $0.5 \mathrm{~mm}$ thick cadmium covers. With the exception of scandium, iron, and cobalt, the irradiation included at least two foils of each material. The measured activities provide an estimate of the reaction rates and fluxes averaged over the foil volumes at the image plane, as described in Equations 3.1 and 3.2:

$$
\begin{gathered}
R=\frac{A}{\left(1-e^{-\lambda t}\right) \cdot V_{f o i l}} \\
\phi=\frac{A}{m \cdot I F} \cdot \frac{M W}{\sigma \cdot N_{A} \cdot\left(1-e^{-\lambda t}\right)}
\end{gathered}
$$

ASTM Standard E261-10 provided the thermal absorption cross sections for the bare foils (ASTM International, 2010a). A modified version of the MCNP beamline model discussed in Section 2.2.6 incorporated the position and composition of each foil. A volume flux tally (type F4) (X-5 Monte Carlo Team, 2003) averaged over each foil estimated the flux per source particle in each foil. An additional flux tally, including the foil's atom density and an energy dependent cross section multiplier, predicts the reaction rate per source particle averaged over each foil. 
Table 3.2. Activation foil masses and post-irradiation activities.

\begin{tabular}{|c|c|c|c|c|}
\hline Foil & Species & Mass $(g)$ & $\begin{array}{c}2011 \text { Activity } \\
(\mu \mathrm{Ci})\end{array}$ & $\begin{array}{c}2012 \text { Activity } \\
(\mu \mathrm{Ci})\end{array}$ \\
\hline Dy-1 & Dy-165 & 0.0312 & 2.4679 & - \\
\hline Dy-2 & Dy-165 & 0.0343 & 2.8331 & - \\
\hline $\ln -1$ & In-116m & 0.107 & 10.1971 & - \\
\hline $\ln -2^{*}$ & In-116m & 0.1564 & 4.5199 & 9.51 \\
\hline $\ln -3$ & $\ln -116 \mathrm{~m}$ & 0.1467 & 13.3056 & 9.30 \\
\hline $\mathrm{MnCu}-1$ & $\mathrm{Mn}-56$ & 0.0477 & 0.2404 & - \\
\hline MnCu-2 & $\mathrm{Mn}-56$ & 0.0480 & 0.1848 & $1.58 \mathrm{E}-1$ \\
\hline $\mathrm{MnCu}-3^{*}$ & $\mathrm{Mn}-56$ & 0.0506 & 0.0536 & $1.89 \mathrm{E}-1$ \\
\hline$A u-1$ & Au-198 & 0.3325 & 0.2403 & $6.38 \mathrm{E}-1$ \\
\hline $\mathrm{Au}-2$ & Au-198 & 0.3304 & 0.2312 & 5.7E-1 \\
\hline $\mathrm{Au}-3^{*}$ & Au-198 & 0.3366 & 0.0881 & - \\
\hline Sc-1 & Sc- -46 & 0.0463 & 0.0008 & $4.95 \mathrm{E}-4$ \\
\hline $\mathrm{Cu}-1$ & Cu-64 & 0.1419 & 0.0373 & $6.41 \mathrm{E}-2$ \\
\hline Cu-2 & Cu-64 & 0.2881 & 0.0726 & $1.21 \mathrm{E}-1$ \\
\hline $\mathrm{Cu}-3^{*}$ & Cu-64 & 0.2873 & 0.0202 & - \\
\hline W-1 & W-187 & 0.0571 & 0.0200 & $9.63 \mathrm{E}-2$ \\
\hline W-2 & W-187 & 0.0582 & 0.0240 & $1.01 \mathrm{E}-1$ \\
\hline Ta-1 & Ta-182 & 0.1037 & - & $5.37 \mathrm{E}-3$ \\
\hline Ta-2 & Ta-182 & 0.1042 & - & $5.25 \mathrm{E}-3$ \\
\hline $\mathrm{Nb}-1$ & $\mathrm{Nb}-93$ & 0.1397 & - & $N / D^{* *}$ \\
\hline $\mathrm{Nb}-2$ & $\mathrm{Nb}-93$ & 0.1395 & - & $N / D^{* *}$ \\
\hline Co-1 & Co- 60 & 0.0623 & - & $1.32 \mathrm{E}-4$ \\
\hline $\mathrm{Fe}-1$ & $\mathrm{Fe}-57$ & 0.1319 & - & $\mathrm{N} / \mathrm{D}^{* *}$ \\
\hline
\end{tabular}

${ }^{*}$ Cadmium covered foil irradiated in 2011

${ }^{* *}$ No detectable activity

\subsubsection{Bare Foil Results}

Table 3.3 shows the MCNP calculated reaction rates and fluxes, the measured reaction rates and fluxes, and the resulting source particle rates for all of the bare foils. The simulation tracked 5 billion particles resulting in tally uncertainties below $10 \%$. Calculating the source particle rate required to produce the observed activation rate in each foil allows the foils to be compared for consistency. The source particle rate (SPR) is an MCNP abstraction and corresponds to the total number of neutrons leaving the aperture per unit time. 
Table 3.3. Reaction rates, fluxes, and source particle rates predicted by MCNP for the bare foils.

\begin{tabular}{|c|c|c|c|c|c|c|}
\hline Foil & $\begin{array}{c}\text { Predicted } \\
\text { Reaction } \\
\text { Rate } \\
\left(\mathrm{rxn} / \mathrm{cm}^{3} \mathrm{sp}\right)\end{array}$ & $\begin{array}{c}\text { Measured } \\
\text { Reaction } \\
\text { Rate } \\
\left(\mathbf{r x n} / \mathrm{cm}^{3} \mathrm{~s}\right)\end{array}$ & $\begin{array}{l}\text { Predicted } \\
\text { Flux } \\
\left(\mathrm{n} / \mathrm{cm}^{2} \mathrm{sp}\right)\end{array}$ & $\begin{array}{l}\text { Measured } \\
\text { Flux } \\
\left(\mathrm{n} / \mathrm{cm}^{2} \mathbf{s}\right)\end{array}$ & $\begin{array}{c}\text { SPR (flux) } \\
\text { (n/s) }\end{array}$ & $\begin{array}{c}\text { SPR } \\
\text { (reaction } \\
\text { rate) } \\
(n / s)\end{array}$ \\
\hline Dy-2 & $3.50 \mathrm{E}-02$ & $3.41 \mathrm{E}+08$ & $3.24 \mathrm{E}-04$ & $4.09 \mathrm{E}+06$ & $1.26 \mathrm{E}+10$ & $9.75 \mathrm{E}+09$ \\
\hline Dy-1 & $3.45 \mathrm{E}-02$ & $3.60 \mathrm{E}+08$ & $3.17 \mathrm{E}-04$ & $4.27 \mathrm{E}+06$ & $1.35 \mathrm{E}+10$ & $1.05 E+10$ \\
\hline In-1 & $3.00 \mathrm{E}-03$ & $5.24 \mathrm{E}+07$ & $3.10 \mathrm{E}-04$ & $7.91 \mathrm{E}+06$ & $2.55 \mathrm{E}+10$ & $1.68 \mathrm{E}+10$ \\
\hline In-3 & $2.85 \mathrm{E}-03$ & $4.99 \mathrm{E}+07$ & $2.98 \mathrm{E}-04$ & $7.53 E+06$ & $2.52 \mathrm{E}+10$ & $1.68 \mathrm{E}+10$ \\
\hline $\mathrm{MnCu}-1$ & 4.48E-04 & $8.41 E+06$ & 3.55E-04 & $6.64 \mathrm{E}+06$ & $1.87 \mathrm{E}+10$ & $1.62 E+10$ \\
\hline MnCu-2 & $4.32 \mathrm{E}-04$ & $6.42 \mathrm{E}+06$ & $3.42 \mathrm{E}-04$ & $5.07 \mathrm{E}+06$ & $1.48 \mathrm{E}+10$ & $1.28 \mathrm{E}+10$ \\
\hline $\mathrm{Au}-1$ & $2.47 \mathrm{E}-03$ & $4.85 \mathrm{E}+07$ & 3.27E-04 & $8.32 \mathrm{E}+06$ & $2.55 \mathrm{E}+10$ & $1.96 \mathrm{E}+10$ \\
\hline Au-2 & 2.53E-03 & $4.70 \mathrm{E}+07$ & 3.35E-04 & $8.06 \mathrm{E}+06$ & $2.41 \mathrm{E}+10$ & $1.86 \mathrm{E}+10$ \\
\hline Sc-1 & 4.17E-04 & $4.83 \mathrm{E}+06$ & $3.44 \mathrm{E}-04$ & $5.32 \mathrm{E}+06$ & $1.54 \mathrm{E}+10$ & $1.16 \mathrm{E}+10$ \\
\hline Cu-1 & $1.40 \mathrm{E}-04$ & $2.83 \mathrm{E}+06$ & $3.28 \mathrm{E}-04$ & $6.25 \mathrm{E}+06$ & $1.91 \mathrm{E}+10$ & $1.70 \mathrm{E}+10$ \\
\hline Cu-2 & 1.44E-04 & $2.71 \mathrm{E}+06$ & $3.38 \mathrm{E}-04$ & $5.99 \mathrm{E}+06$ & $1.77 \mathrm{E}+10$ & $1.58 \mathrm{E}+10$ \\
\hline W-1 & $1.11 \mathrm{E}-03$ & $3.07 \mathrm{E}+07$ & $3.51 \mathrm{E}-04$ & $1.30 \mathrm{E}+07$ & $3.71 \mathrm{E}+10$ & $2.76 \mathrm{E}+10$ \\
\hline \multirow[t]{2}{*}{ W-2 } & $1.08 \mathrm{E}-03$ & $3.61 \mathrm{E}+07$ & 3.44E-04 & $1.53 E+07$ & $4.46 \mathrm{E}+10$ & $3.33 \mathrm{E}+10$ \\
\hline & & & Average & $7.52 \mathrm{E}+06$ & $2.26 \mathrm{E}+10$ & $1.74 \mathrm{E}+10$ \\
\hline
\end{tabular}

The source particle rate for this simulation is simply the ratio of the measured and predicted quantities:

$$
S P R=\frac{\varphi_{\text {Experimental }}}{\varphi_{M C N P}} \text { or } \frac{R_{\text {Experimental }}}{R_{M C N P}}
$$

If the source particle rates calculated for each foil agree with each other, the model can be assumed to reasonably predict the energy spectrum corresponding to the peak absorption regions of each foil.

Figure 3.10 presents the source particle rates calculated for the bare foils in the 2011 irradiation. The square data points represent source particle rates calculated using fluxes, based on mono-energetic cross sections from literature (Pritychenko, et al., 2006). The top line is the average of the source particle rates calculated using the flux data. The diamond data points represent source particle rates calculated based on reaction rate data. The bottom line is the average value of the source particle rates calculated using the reaction rate data. The error bars correspond to a $95.4 \%(2 \sigma)$ confidence interval. 


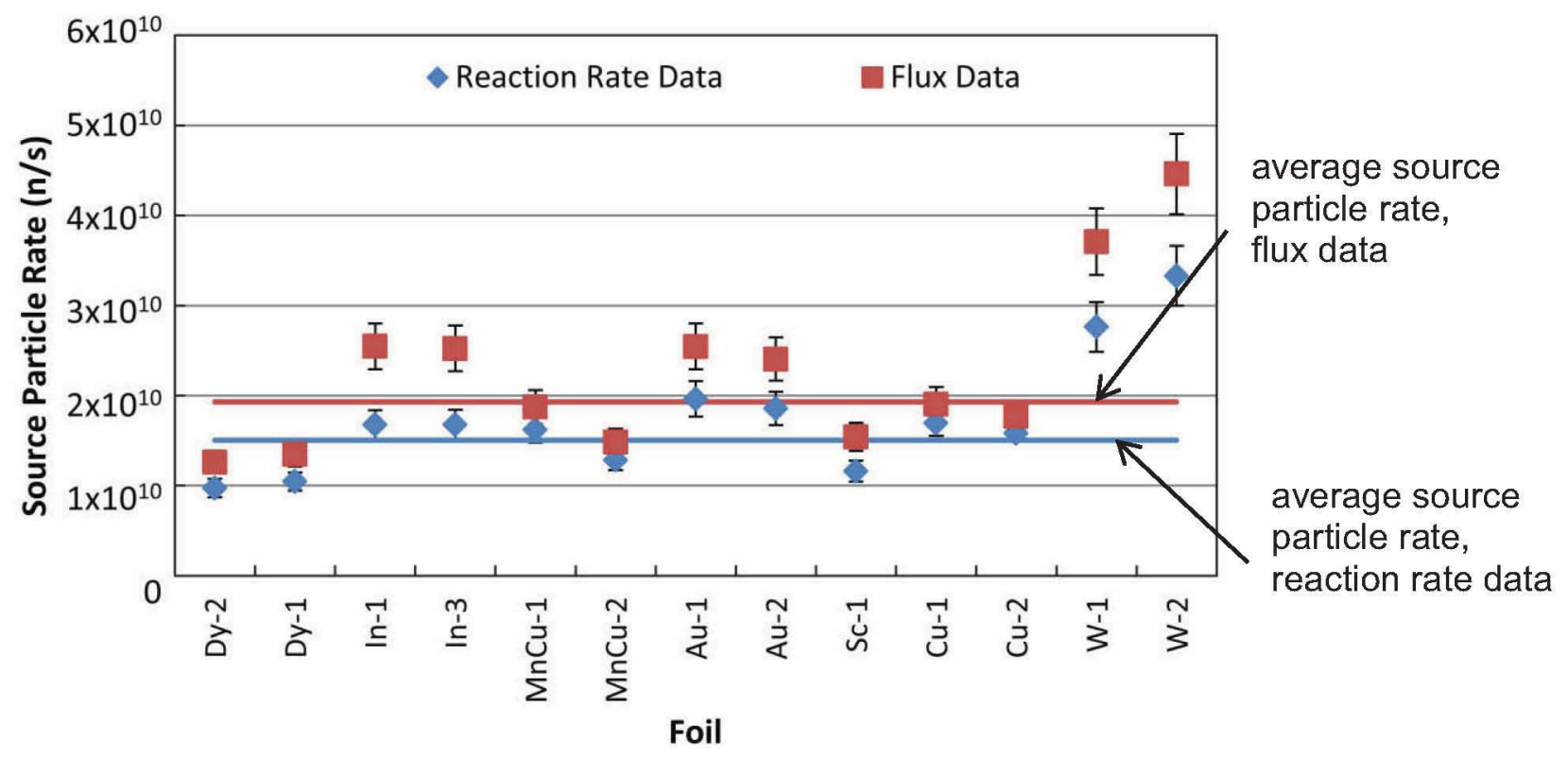

Figure 3.10. Calculated source particle rate for the bare foils.

The reaction rate calculations include the energy-dependent cross section data from MCNP. MCNP uses the energy dependent cross section libraries for each interaction; the NRAD beamline model uses ENDF/B-VII cross sections (Chadwick et al., 2006). The reaction rates were calculated by multiplying the MCNP tally result by the atom density of the foil and by the energy-dependent absorption reaction cross section. The source particle rates agree relatively well among the bare foils, with the exception of tungsten.

\subsubsection{Cadmium Covered Foil Results}

Figure 3.11 shows the cadmium covered source particle rates from the July 31, 2011 irradiations calculated using both literature and MCNP generated cross sections. Figure 3.12 shows the source particle rates calculated for the cadmium covered foils irradiated on October 16, 2012. Table 3.4 presents the cadmium covered fluxes, reaction rates, and source particle rates for both irradiations. The source particle rates calculated for the cadmium covered foils are an order of magnitude higher than those calculated for the bare foils. 


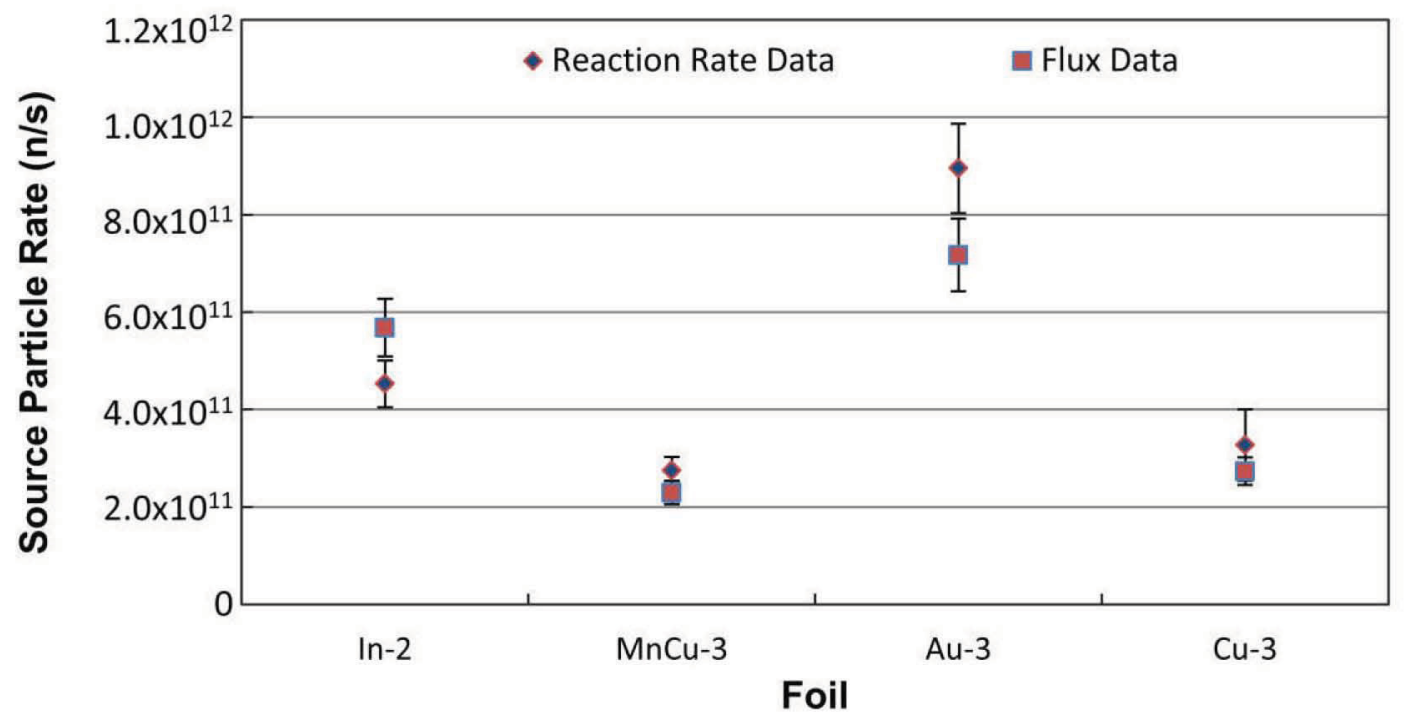

Figure 3.11. Calculated source particle rates for the cadmium covered foils irradiated on July 31, 2011.

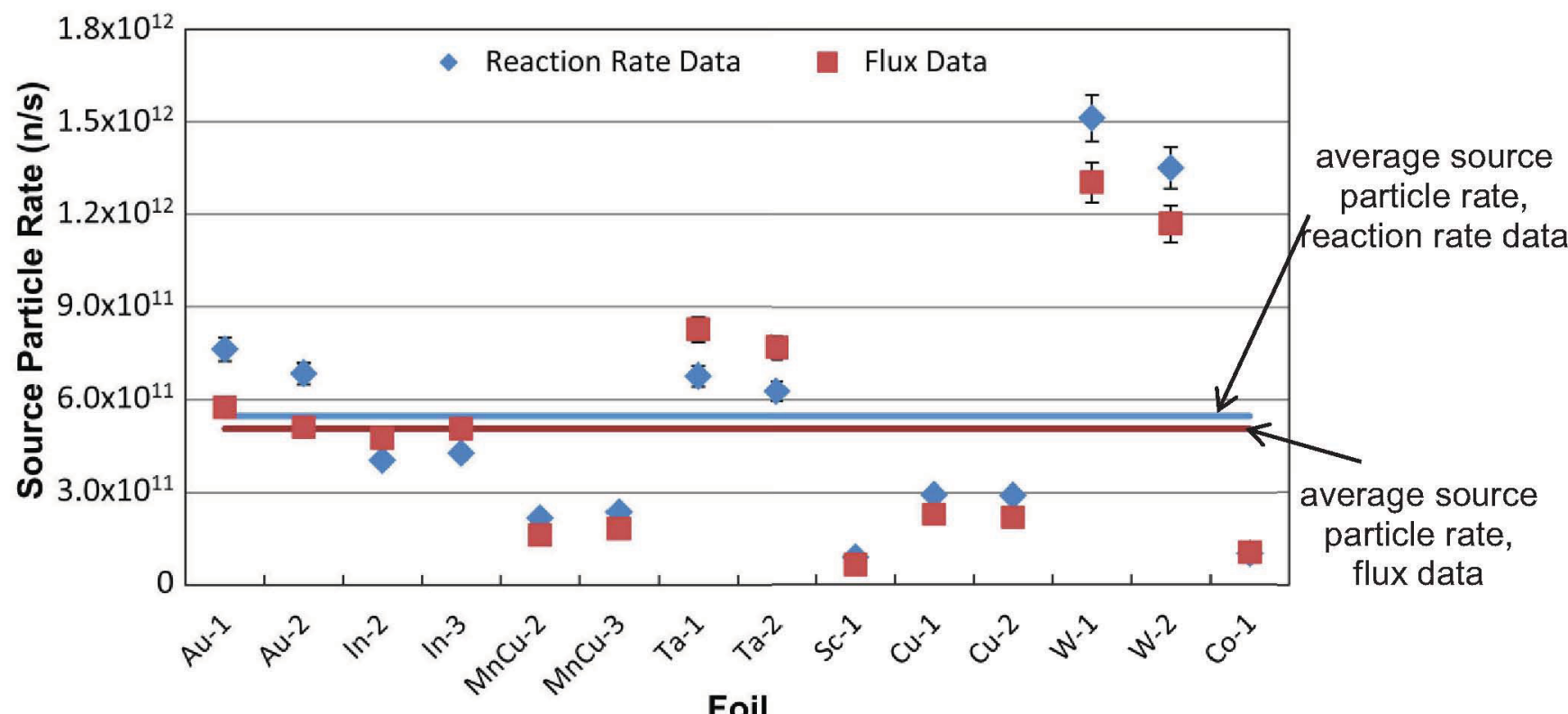

Figure 3.12. Calculated source particle rates for cadmium covered foils irradiated on October 16, 2012.

The data from the foil irradiations suggests that the model spectrum under- or oversamples the flux in the epithermal and fast regions of the spectrum. If the thermal region is assumed to be accurate, then the model underpredicts the flux in the fast region; however, if the 
Table 3.4. Cadmium covered foil fluxes and source particle rates.

\begin{tabular}{|c|c|c|c|c|c|c|}
\hline Foil & $\begin{array}{l}\text { Predicted } \\
\text { Reaction } \\
\text { Rate } \\
\left(\mathbf{r x n} / \mathrm{cm}^{3} \mathrm{sp}\right)\end{array}$ & $\begin{array}{l}\text { Measured } \\
\text { Reaction } \\
\text { Rate } \\
\left(\mathbf{r x n} / \mathrm{cm}^{3} \mathrm{~s}\right)\end{array}$ & $\begin{array}{c}\text { MCNP } \\
\text { Calculated } \\
\text { Foil } \\
\text { Averaged } \\
\text { Flux } \\
\left(\mathrm{n} / \mathrm{cm}^{2} \mathrm{sp}\right) \\
\end{array}$ & $\begin{array}{c}\text { Experimental } \\
\text { Foil Averaged } \\
\text { Flux } \\
\left(\mathrm{n} / \mathrm{cm}^{2} \mathrm{~s}\right)\end{array}$ & $\begin{array}{c}\text { SPR (flux) } \\
(n / s)\end{array}$ & $\begin{array}{c}\text { SPR } \\
\text { (Rxn rate) } \\
(\mathrm{n} / \mathrm{s})\end{array}$ \\
\hline $\mathrm{Au}-1$ & $3.50 E-02$ & $1.67 E+07$ & 4.99E-06 & $2.86 \mathrm{E}+06$ & $5.74 \mathrm{E}+11$ & $7.61 \mathrm{E}+11$ \\
\hline $\mathrm{Au}-2$ & 3.45E-02 & $1.50 \mathrm{E}+07$ & $5.05 E-06$ & $2.58 \mathrm{E}+06$ & $5.09 \mathrm{E}+11$ & $6.84 E+11$ \\
\hline $\ln -2$ & 3.00E-03 & $1.65 \mathrm{E}+07$ & 4.68E-06 & $2.22 \mathrm{E}+06$ & $4.74 \mathrm{E}+11$ & $4.03 E+11$ \\
\hline $\ln -3$ & 2.85E-03 & $1.72 E+07$ & 4.59E-06 & $2.32 E+06$ & $5.05 E+11$ & $4.25 E+11$ \\
\hline MnCu-2 & 4.48E-04 & $1.02 E+06$ & $7.16 \mathrm{E}-06$ & $1.16 \mathrm{E}+06$ & $1.61 \mathrm{E}+11$ & $2.16 \mathrm{E}+11$ \\
\hline $\mathrm{MnCu}-3$ & 4.32E-04 & $1.16 \mathrm{E}+06$ & $7.24 \mathrm{E}-06$ & $1.31 \mathrm{E}+06$ & $1.81 \mathrm{E}+11$ & $2.35 E+11$ \\
\hline Ta-1 & $2.47 \mathrm{E}-03$ & $5.50 \mathrm{E}+06$ & $1.64 \mathrm{E}-05$ & $1.35 \mathrm{E}+07$ & $8.25 E+11$ & $6.76 E+11$ \\
\hline Ta-2 & 2.53E-03 & $5.59 E+06$ & 1.79E-05 & $1.37 \mathrm{E}+07$ & $7.68 \mathrm{E}+11$ & $6.27 E+11$ \\
\hline $\mathrm{Sc}-1$ & 4.17E-04 & $3.59 \mathrm{E}+05$ & 6.29E-06 & $3.95 \mathrm{E}+05$ & $6.27 E+10$ & $8.65 E+10$ \\
\hline $\mathrm{Cu}-1$ & 1.40E-04 & $4.23 E+05$ & 7.11E-06 & $1.61 \mathrm{E}+06$ & $2.26 \mathrm{E}+11$ & $2.91 E+11$ \\
\hline Cu-2 & 1.44E-04 & $3.94 \mathrm{E}+05$ & $6.90 \mathrm{E}-06$ & $1.50 \mathrm{E}+06$ & $2.17 E+11$ & $2.89 E+11$ \\
\hline W-1 & 1.11E-03 & $6.07 E+06$ & 6.96E-06 & $9.06 \mathrm{E}+06$ & $1.30 E+12$ & $1.51 E+12$ \\
\hline W-2 & $1.08 \mathrm{E}-03$ & $5.68 \mathrm{E}+06$ & $7.25 \mathrm{E}-06$ & $8.48 E+06$ & 1.16E+12 & $1.35 E+12$ \\
\hline Co-1 & 5.90E-05 & $5.81 E+06$ & 1.67E-05 & $1.72 \mathrm{E}+06$ & $1.02 E+11$ & $9.85 E+10$ \\
\hline $\ln -2^{*}$ & $3.24 \mathrm{E}-05$ & $1.52 E+07$ & 3.47E-06 & $1.57 \mathrm{E}+06$ & $4.53 E+11$ & $4.53 E+11$ \\
\hline $\mathrm{MnCu}-3^{*}$ & 4.80E-06 & $1.53 E+06$ & 6.09E-06 & 1.67E+06 & $2.74 \mathrm{E}+11$ & $2.74 \mathrm{E}+11$ \\
\hline $\mathrm{Au}-3^{*}$ & 1.96E-05 & $1.76 \mathrm{E}+07$ & 4.20E-06 & $3.76 \mathrm{E}+06$ & $8.95 E+11$ & $8.95 E+11$ \\
\hline \multirow[t]{2}{*}{$\mathrm{Cu}-3^{*}$} & 1.63E-06 & $6.36 \mathrm{E}+05$ & $6.13 \mathrm{E}-06$ & $2.01 E+06$ & 3.27E+11 & $3.27 E+11$ \\
\hline & & & Average & $4.46 \mathrm{E}+06$ & $4.76 \mathrm{E}+11$ & $5.17 \mathrm{E}+11$ \\
\hline
\end{tabular}

*Irradiated in 2011.

fast region is assumed to be accurate, then the model overpredicts the flux in the thermal region. This explains the difference in calculated source particle rates between the thermal and fast regions, but does not necessarily explain which region is correctly or incorrectly modeled.

The MCNP model predicts a gold foil cadmium ratio of 68.5 based on the average of all calculated gold foil fluxes. The cadmium ratios calculated from the 2011 and 2012 measured flux data are more than an order of magnitude lower than the cadmium ratios calculated using the MCNP generated flux data. The cadmium ratio calculated from the 2011 gold foil irradiation data is 2.7 . The cadmium ratio calculated using the gold foils from the 2012 irradiations is 2.8 . 
This indicates that the model spectrum oversamples the thermal region. The spectrum in the NRAD beamline model is based on the highly enriched core (Imel and Urbatsch, 1992), and neglects the resonance region absorption by the U-238 currently in the low-enriched core. This implies that the model spectrum overestimates the ratio of thermal to fast neutrons in the neutron beam. Resolving this discrepancy will require the creation of a new model spectrum, which is outside scope of this project, but is recommended for a future project.

In both the thermal and cadmium covered foil results, tungsten is a clear outlier. This may be due to inaccuracies in the composition of the tungsten foils. This may also be due to the lack of cross section data for W-180 in the MCNP libraries. The following section presents the results of measuring the flux profile of the NRAD beam.

\subsection{Flux Profile}

The blank dysprosium and indium radiographs shown in Figure 3.13 were taken on August 1, 2011, digitized using a Fuji FineScan 1500 film scanner, and imported into the ImageJ graphics editing program (http://rsb.info.nih.gov/ij/). The film is the standard size for a NRAD radiograph (7 inches wide by 17 inches tall) (Davidson and Kahn, 2006). Figure 3.14 shows a normalized gray-value profile across the top middle of the indium and dysprosium radiographs. The positions at which the profiles were taken are indicated on Figures 3.13.a and 3.13.b. Lower pixel values in Figure 3.14 represent higher neutron flux values. The figure indicates a slightly asymmetric beam cross section, most likely due to asymmetry in the core of the NRAD.

The NRAD also irradiated an array of gold and dysprosium foils for 20 minutes on October 17, 2012. Once the foils were counted, the flux averaged across each foil could be calculated using literature cross sections. A modified MCNP model of the beamline simulated the experiment, placing gold and dysprosium foils in the same locations relative to the image plane as in the model. Figure 3.15 depicts the positions of the gold foils. Table 3.5 compares the calculated flux values to measured values. 


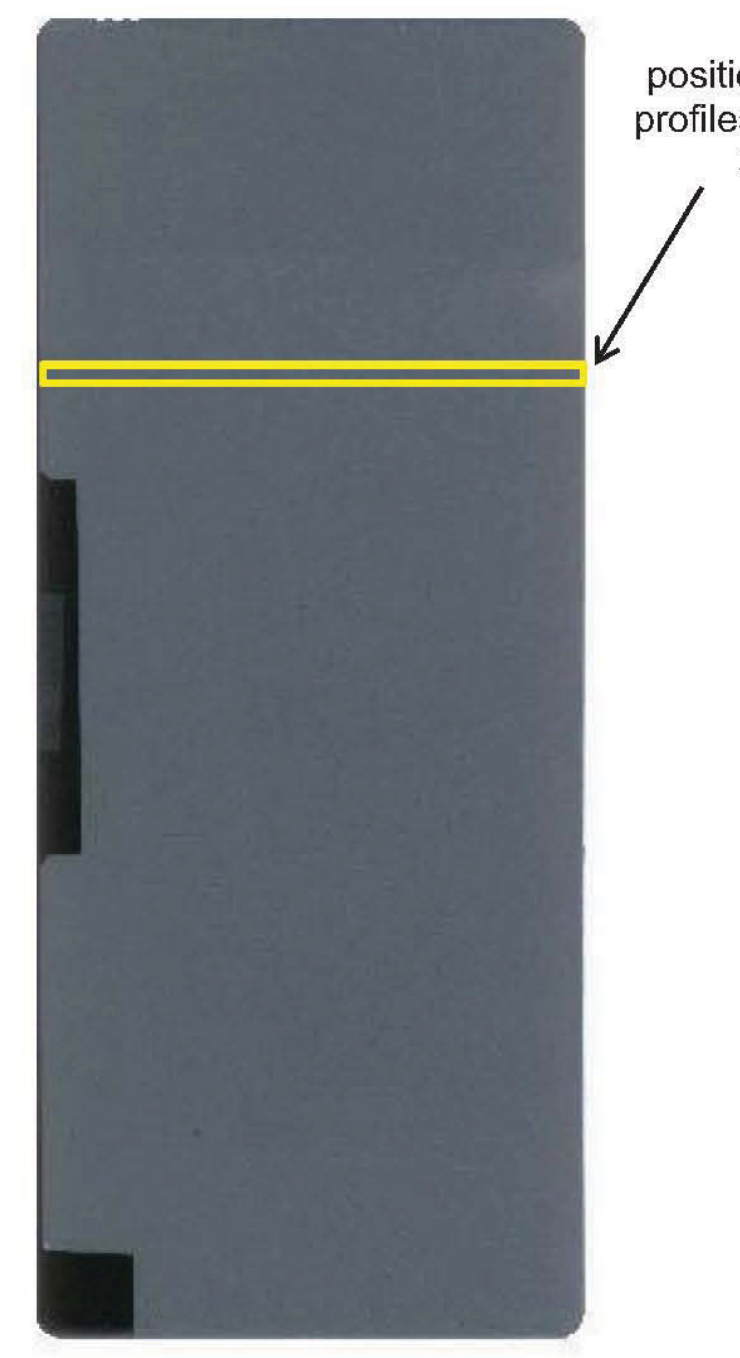

(a) indium radiograph
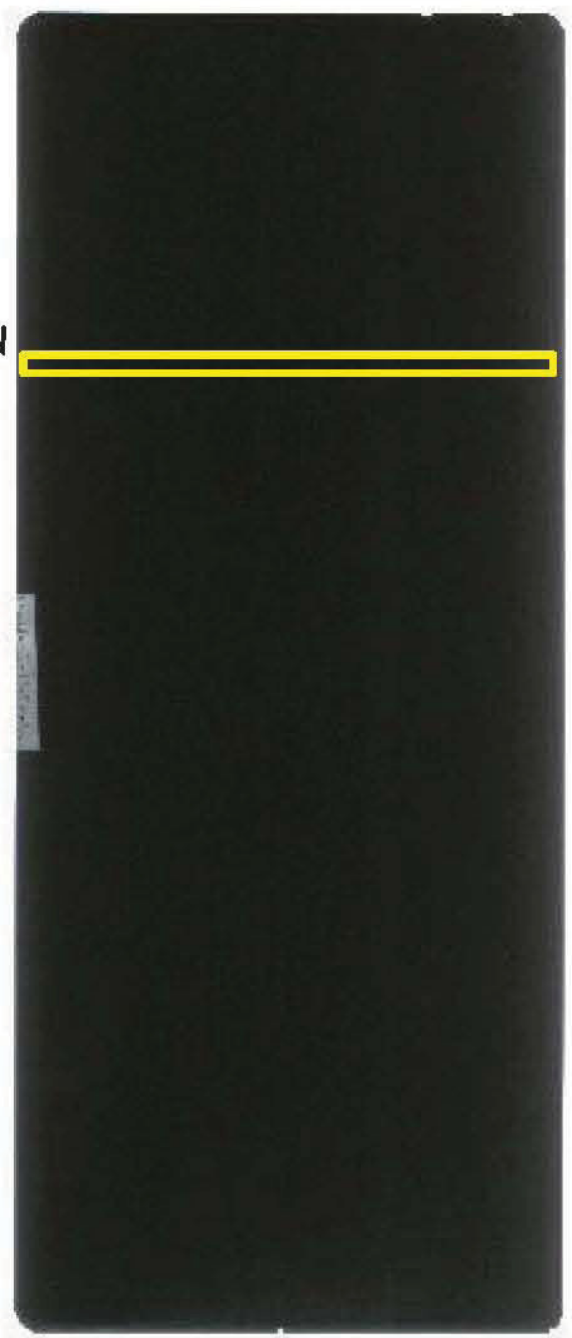

(b) dysprosium radiograph

Figure 3.13. Blank indium and dysprosium radiographs.

The peak to average flux values consider either gold or dysprosium data and show that there is little variation across the image plane. The gold and dysprosium data sets are consistent within themselves. The calculated source particle rates agree well with the source particle rates calculated using the bare foil activation data from 2011. The average source particle rate from the 2011 bare foil data was $2.26 \times 10^{10} \mathrm{n} / \mathrm{s}$ and the average source particle rate from the 2012 gold and dysprosium array data was $1.95 \times 10^{10} \mathrm{n} / \mathrm{s}$ (see Table 3.5).

Dysprosium has a high neutron absorbtion cross section which causes the flux within a dysprosium foil to be lower than the flux in the surrounding area. This causes the measured flux 


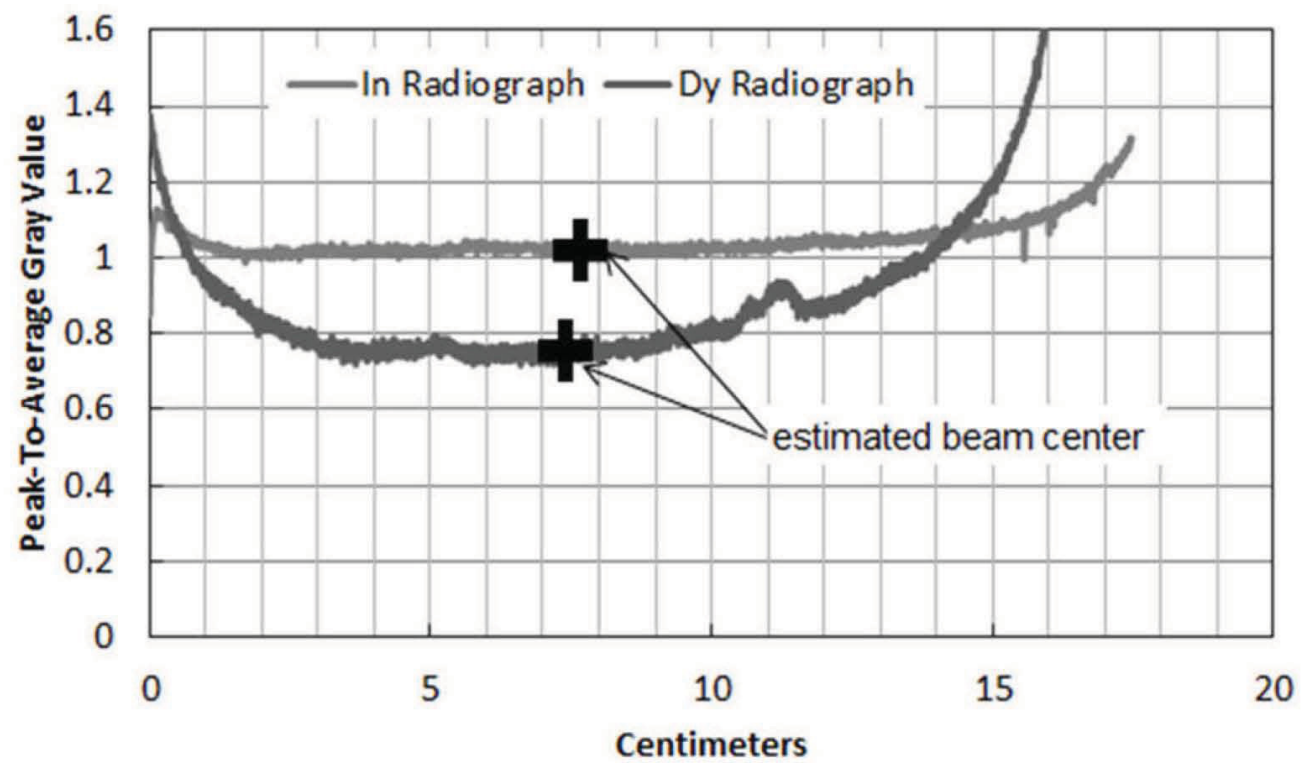

Figure 3.14. Normalized gray values across the dysprosium and indium foil radiographs.

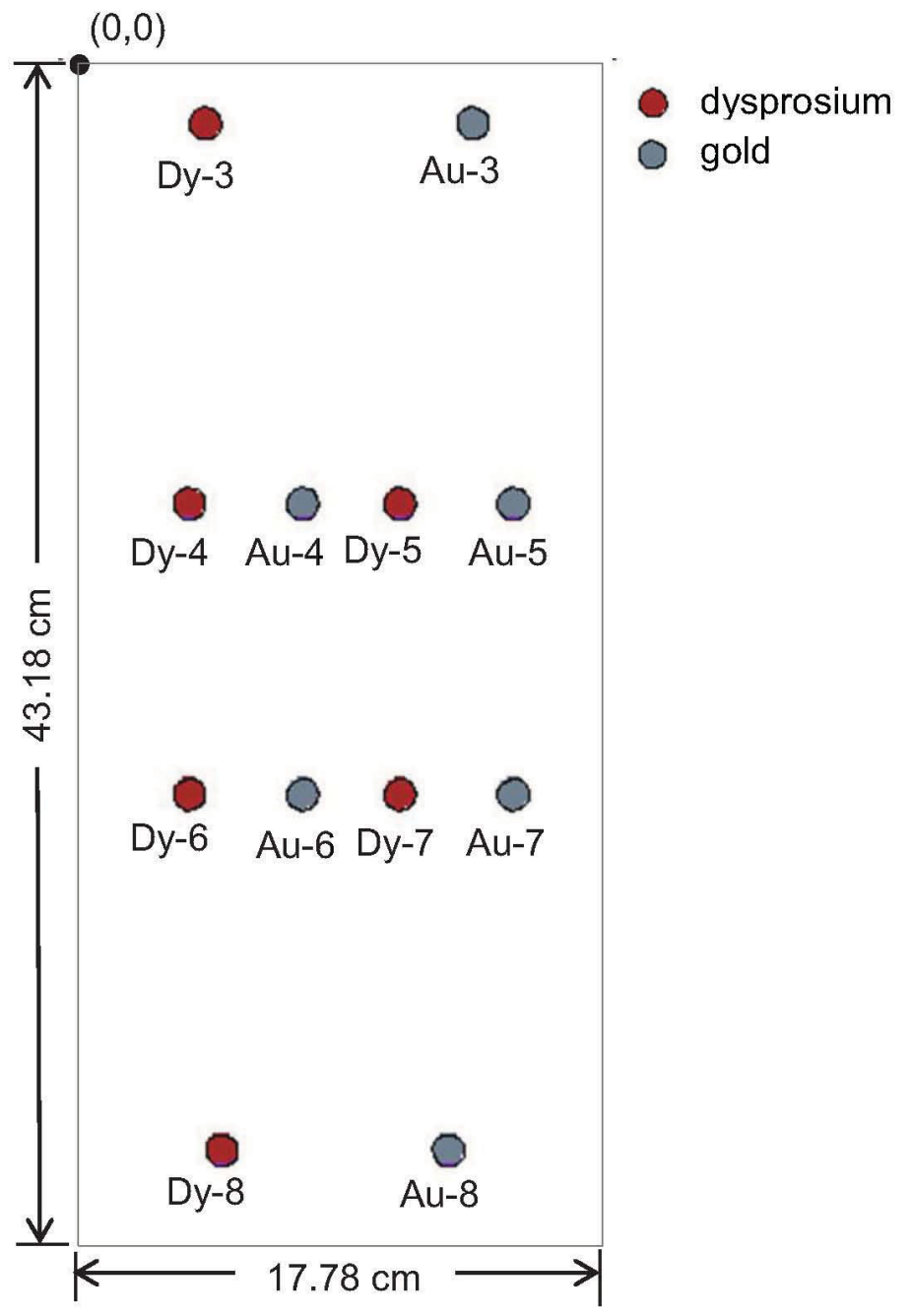

Figure 3.15. Positions of gold and dysprosium foils on the image plane. 
Table 3.5. MCNP calculated fluxes and fluxes calculated from foil activity.

\begin{tabular}{cccccc}
\hline Position from & $\begin{array}{c}\text { MCNP } \\
(\mathbf{0}, \mathbf{0})\end{array}$ & $\begin{array}{c}\text { Calculated Foil } \\
\text { Averaged Flux } \\
\left(\mathbf{n} / \mathbf{c m}^{2} \mathbf{s p}\right)\end{array}$ & $\begin{array}{c}\text { Experimentally } \\
\text { Measured Foil } \\
\text { Averaged Flux } \\
\left(\mathbf{n} / \mathbf{c m}^{2} \mathbf{s}\right)\end{array}$ & $\begin{array}{c}\text { Peak to } \\
\text { Average } \\
\text { Ratio }\end{array}$ & $\begin{array}{c}\text { Source } \\
\text { Particle Rate } \\
\text { (n/s) }\end{array}$ \\
\hline Dy-3 & $(3.81,-1.59)$ & $3.04 \times 10^{-4}$ & $3.68 \times 10^{6}$ & 0.95 & $9.08 \times 10^{9}$ \\
Dy-4 & $(3.175,-16.5)$ & $2.34 \times 10^{-4}$ & $3.74 \times 10^{6}$ & 0.97 & $1.43 \times 10^{10}$ \\
Dy-5 & $(11.43,-16.5)$ & $3.01 \times 10^{-4}$ & $4.01 \times 10^{6}$ & 1.03 & $1.08 \times 10^{10}$ \\
Dy-6 & $(3.175,-27.94)$ & $2.37 \times 10^{-4}$ & $4.03 \times 10^{6}$ & 1.04 & $1.51 \times 10^{10}$ \\
Dy-7 & $(11.43,-27.94)$ & $2.96 \times 10^{-4}$ & $3.89 \times 10^{6}$ & 1.00 & $1.07 \times 10^{10}$ \\
Dy-8 & $(4.445,-41.91)$ & $2.19 \times 10^{-4}$ & $3.91 \times 10^{6}$ & 1.01 & $1.50 \times 10^{10}$ \\
Au-3 & $(14.2875,-1.59)$ & $3.07 \times 10^{-4}$ & $7.36 \times 10^{6}$ & 0.95 & $1.80 \times 10^{10}$ \\
Au-4 & $(7.62,-16.5)$ & $3.39 \times 10^{-4}$ & $7.81 \times 10^{6}$ & 1.00 & $1.76 \times 10^{10}$ \\
Au-5 & $(15.875,-16.5)$ & $3.26 \times 10^{-4}$ & $7.87 \times 10^{6}$ & 1.01 & $1.84 \times 10^{10}$ \\
Au-6 & $(7.62,-27.94)$ & $3.36 \times 10^{-4}$ & $7.88 \times 10^{6}$ & 1.01 & $1.79 \times 10^{10}$ \\
Au-7 & $(15.558,-27.94)$ & $3.26 \times 10^{-4}$ & $7.84 \times 10^{6}$ & 1.01 & $1.83 \times 10^{10}$ \\
Au-8 & $(13.335,-41.91)$ & $3.08 \times 10^{-4}$ & $7.95 \times 10^{6}$ & 1.02 & $1.94 \times 10^{10}$ \\
\hline & & & $5.83 \times 10^{6}$ & Average & $1.95 \times 10^{10}$ \\
\hline
\end{tabular}

within the dysprosium foils to be lower than the flux measured within the gold foils. Gold does not have as high of a cross section as dysprosium, and so the self-shielding effect is less significant.

\subsection{Flux Estimates}

Modeling the array of gold and dysprosium foils using void filled foil cells and a fluence (F2) tally at the image plane predicts the flux at the image plane without self-shielding effects. Table 3.6 displays the fluxes calculated by the MCNP model of the NRAD beamline for the thermal, resonance, and fast regions of the spectrum, using the source particle rate determined by both the bare foil activation results in Table 3.3 , and the cadmium covered foil activation results in Table 3.4 .

The source particle rates calculated from the cadmium covered foils likely over-estimate the beam flux. The calculated flux at the image plane based on the SPR from the bare foil activation data in Table 3.3 is $5.54 \times 10^{6} \mathrm{n} / \mathrm{cm}^{2} \mathrm{~s}\left( \pm 5.5 \times 10^{5} \mathrm{n} / \mathrm{cm}^{2} \mathrm{~s}\right)$, which is closer to the average flux values calculated in Tables $3.3,3.4$, and $3.5\left(7.52 \times 10^{6}, 4.46 \times 10^{6}\right.$, and $5.83 \times 10^{6} \mathrm{n} / \mathrm{cm}^{2} \mathrm{~s}$, respectively). 
Table 3.6. Thermal, resonance, and fast fluxes predicted by the MCNP model.

\begin{tabular}{ccccc}
\hline $\begin{array}{c}\text { Source } \\
\text { Particle Rate } \\
(\mathbf{n} / \mathbf{s})\end{array}$ & $\begin{array}{c}\text { Average Thermal } \\
\text { Flux [0-0.5eV] } \\
\left(\mathbf{n} / \mathbf{c m}^{2} \mathbf{s}\right)\end{array}$ & $\begin{array}{c}\text { Average Resonance } \\
\text { Flux }[\mathbf{0 . 5 e V - 0 . 1 M e V ]} \\
\left(\mathbf{n} / \mathbf{c m}^{2} \mathbf{s}\right)\end{array}$ & $\begin{array}{c}\text { Average Fast } \\
\text { Flux }[\mathbf{0 . 1 M e V}- \\
10 M e V] \\
\left(\mathbf{n} / \mathbf{c m}^{2} \mathbf{s}\right)\end{array}$ & $\begin{array}{c}\text { Total Flux } \\
\left(\mathbf{n} / \mathbf{c m}^{2} \mathbf{s}\right)\end{array}$ \\
\hline $1.74 \mathrm{E}+10$ & $5.50 \mathrm{E}+06$ & $3.77 \mathrm{E}+04$ & $8.34 \mathrm{E}-01$ & $5.54 \mathrm{E}+06$ \\
\hline $5.17 \mathrm{E}+11$ & $1.74 \mathrm{E}+08$ & $1.19 \mathrm{E}+06$ & $2.05 \mathrm{E}+01$ & $1.75 \mathrm{E}+08$ \\
\hline
\end{tabular}

\subsection{Summary of Results}

The NRAD has an L/D greater than 125 and is a category I radiographic facility. The divergence of the NRAD neutron beam is $0.3 \pm 0.1$ degrees.

The foil activation and flux profile experiments provide a partial validation of the MCNP model of the neutron beamline. The source particle rates calculated for the bare foils are consistent across all of the bare foils. The source particle rates calculated for the cadmium covered foils are consistent across all of the cadmium covered foils. The bare foil results in 2011 predict a source particle rate of $1.74 \times 10^{10} \mathrm{n} / \mathrm{s}$, while the cadmium covered foils from 2011 and 2012 predict a source particle rate of $5.17 \times 10^{11} \mathrm{n} / \mathrm{s}$. The gold foil cadmium ratios for the 2011 and 2012 gold foil irradiations are 2.7 and 2.8 respectively. The MCNP model predicts an average flux of $5.54 \times 10^{6} \mathrm{n} / \mathrm{cm}^{2} \mathrm{~s}$ across the image plane based on bare source particle rate.

The difference in enrichment between the highly enriched and low enriched cores may cause either the thermal or fast regions to be inaccurately modeled in the current NRAD beamline model, as the neutron source is based on the highly enriched core. It is likely that the thermal region is over-sampled in the current NRAD beamline model spectrum.

Chapter 4 discusses the development and validation of the image simulation program to simulate the radiography process at NRAD. 


\section{CHAPTER 4}

\section{IMAGE SIMULATION RESULTS}

The previous chapter provided a full characterization of the NRAD east beamline, along with a partial validation of the MCNP model of the NRAD beamline. This chapter presents a methodology to accurately simulate radiographs produced by the NRAD. Mesh tallies at the image plane in a beamline model can provide raw data to simulate radiograph images. These raw data are not accurate representations of radiographs, however, because simply assigning gray values to flux data using a linear conversion does not take into account film response or scanner calibration. This chapter describes the radiograph simulation program developed for this project, the experimental procedure used to obtain a new characteristic curve for industrial X-ray film, and a comparison of simulated and actual radiographs.

\subsection{Image simulation}

The following section describes the process of developing a radiograph simulator for the NRAD. The simulation works with the MCNP model of the NRAD to produce 8-bit gray value images of objects of interest. The simulation required the development of a new characteristic curve to properly account for film response to radiation emitted by an activated metal foil.

\subsubsection{Data Conversion}

A mesh tally taken at the image plane in the MCNP model provides data for the image simulation seen in Figure 4.1. A MCNP mesh tally estimates the flux over each volume in a three dimensional grid defined by the user. The volumes are right quadrilaterals and each volume is treated as a separate F4 tally. A C++ program (listed in Appendix B) translates the tally data to a gray value image which can be read by ImageJ (http://rsb.info.nih.gov/ij/). The initial prototype of the program used a simple inverted linear interpolation using the tally data to calculate the image gray values (GV) from 0 to 255 (Equation 4.1): 


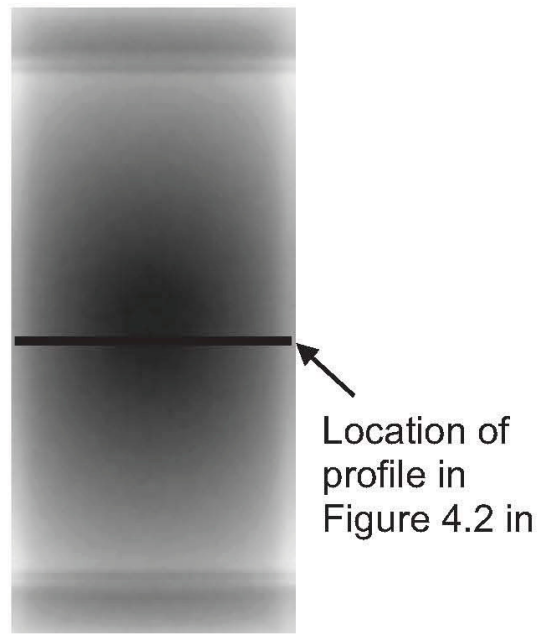

Figure 4.1. Initial grayscale image calculated by the NRAD MCNP beamline model.

$$
G V=256-256\left(\frac{p-p_{\min }}{p_{\max }-p_{\min }}\right) .
$$

In order for lower gray values to correspond to higher neutron fluxes as they do in Figure 3.10, the MCNP data must be inverted to match the radiographs. The simulated profile shown in Figure 4.2 uses a peak-to-average normalization scheme, and is taken at the same relative location in Figure 4.1 as the profiles from Figures 3.14 and 3.15. The curve calculated by the uncorrected simulation has a clearly different shape than that of the radiographs. The actual radiographs have a nearly flat profile, while the model results are much more peaked. This is due to the unknown response of the film to exposure to beta and gamma radiation as well as the non-linearity of the optical density to gray value conversion performed by the scanner. Thus, correction curves that incorporate the actual response of the film and scanner are needed to accurately simulate radiographic images. 


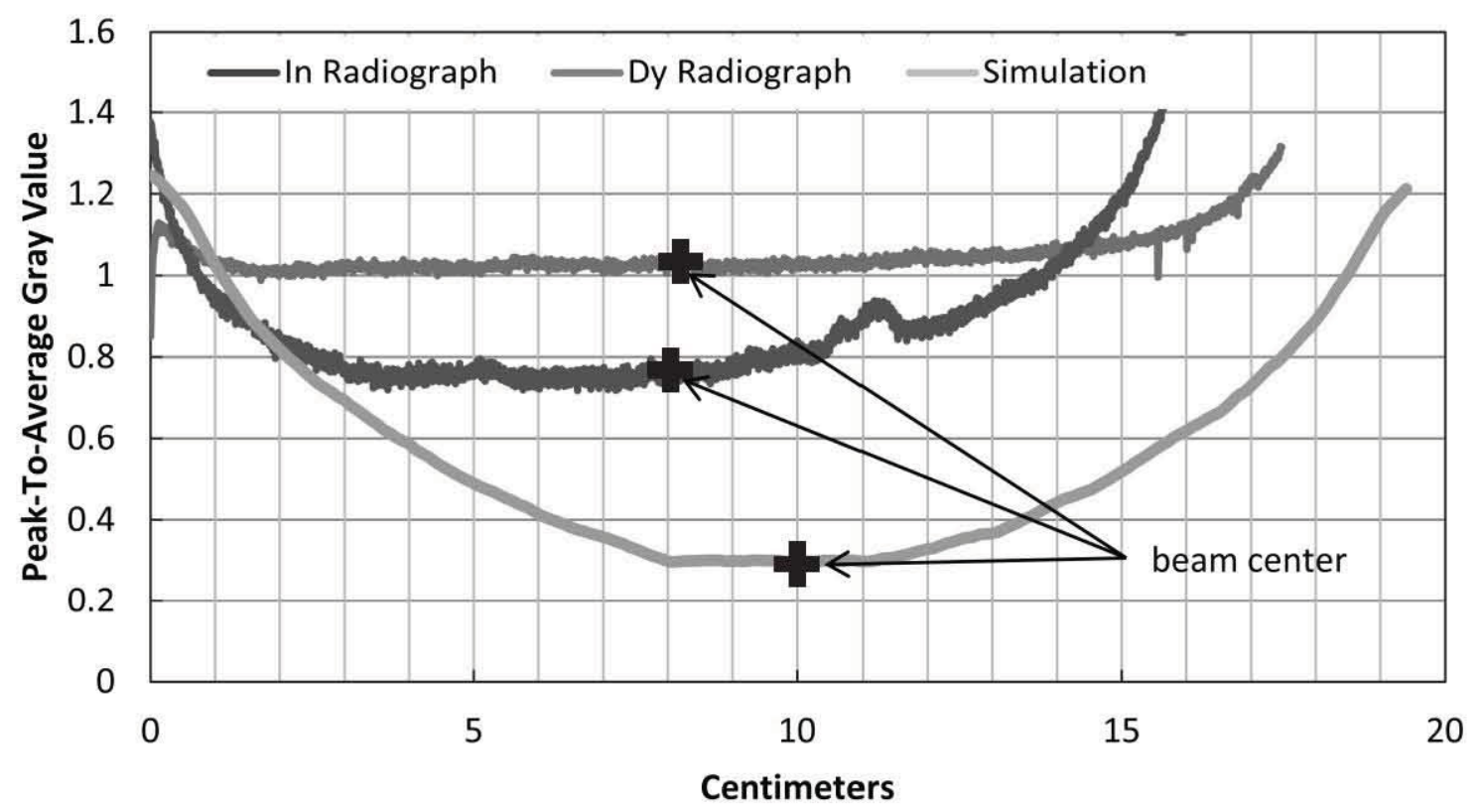

Figure 4.2. Flux profile of a simulated image compared to actual radiographs.

\subsubsection{Development of a Film Characteristic Curve}

To relate activity to optical density, the NRAD irradiated four sets of two dysprosium foils for different amounts of time in the east neutron beam (15, 18, 22, and 27 minutes). The standard shot time for a radiograph at the NRAD is 22 minutes. One foil in each set was a standard imaging foil, 7 inches $\times 17$ inches $\times 0.004$ inches in thickness, and the other was a 0.5 inch diameter, 0.004 inch thick circular foil placed in front of the imaging foil. A gamma spectrometer measured the activity in the small foils while the large foil produced a full size radiograph using the standard development methods. Table 4.1 shows the small foil activities from each irradiation time corrected to activity at beginning of film exposure and the resulting average optical density values on the surrounding area of the larger foil.

Plotting the optical density of the larger foil as a function of the activity of the smaller foil generates a new characteristic curve. Figure 4.3 shows the four points obtained in this experiment plotted with independent activity and dependent optical density. 
Table 4.1. Irradiation results used to produce the characteristic curve in Figure 4.3.

\begin{tabular}{ccc}
\hline $\begin{array}{c}\text { Foil Irradiation Time in } \\
\text { Minutes }\end{array}$ & $\begin{array}{c}\text { Small Foil Activity } \\
\left(\boldsymbol{\mu C i} / \mathbf{c m}^{2}\right)\end{array}$ & $\begin{array}{c}\text { Large Foil Average Optical } \\
\text { Density }\end{array}$ \\
\hline 15 & $0.59 \pm 0.02$ & $2.10 \pm 0.02$ \\
18 & $0.73 \pm 0.02$ & $2.52 \pm 0.02$ \\
22 & $0.81 \pm 0.02$ & $2.81 \pm 0.02$ \\
27 & $0.95 \pm 0.03$ & $3.47 \pm 0.02$ \\
\hline
\end{tabular}

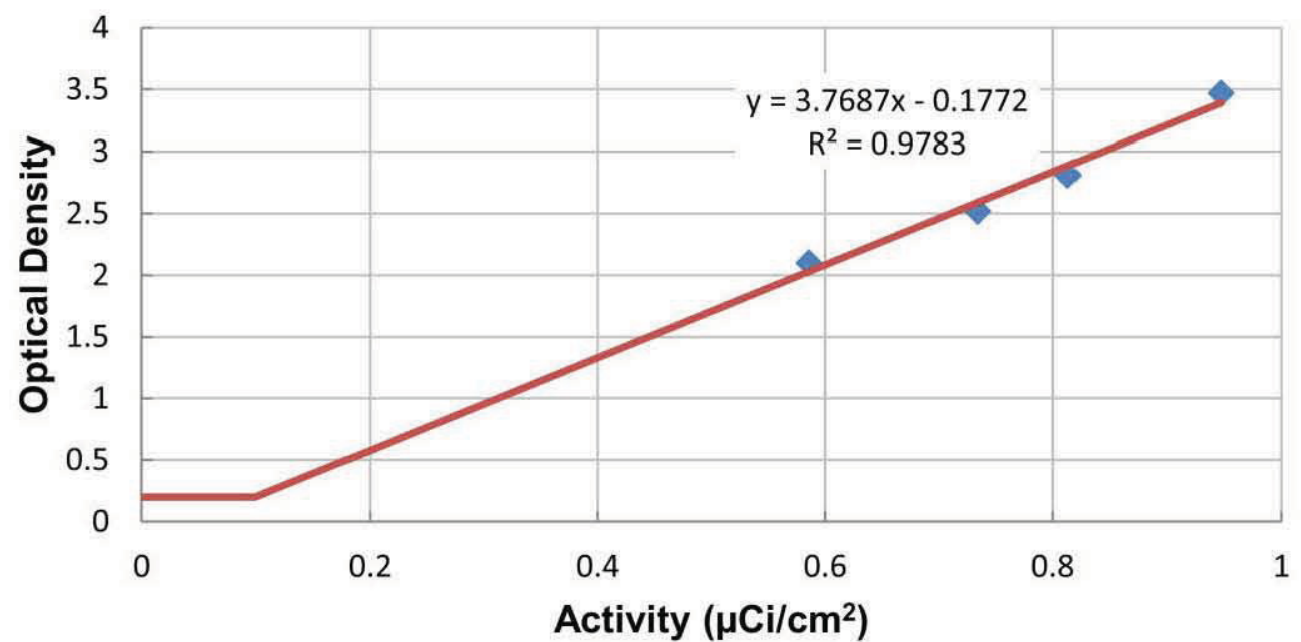

Figure 4.3. Characteristic curve for AGFA D3 s.c. film exposed by activated dysprosium foil.

Fitting a linear equation to the data provides the characteristic curve for the film from an optical density of 0.2 to 3.5 . The curve likely follows an exponential path from 0.2 towards the linear portion of the graph, similar the characteristic curves shown in Section 2.1.3. There are too few data points to accurately estimate the nonlinear region of the curve, so the simulation relies on an linear extrapolation down to the optical density of 0.2 from the 4 known points. The following section explains the structure of the simulation as well and how it utilizes the characteristic curve.

\subsubsection{Implementation of the Simulation}

The image simulation program is written in $\mathrm{C}++$ and can be easily modified to model any metal foil. The simulation outputs an image file in portable gray map (.pgm) format which is a prediction of the image produced when a film radiograph produced by the transfer method is 
scanned into a computer. The simulation models the activation of the foil, the foil decay in the time between foil irradiation and film exposure, the film response to the level of activity in the foil, and the response curve of the film scanner which converts optical densities to grayscale values.

The simulation begins by parsing mesh tally data from the MCNP mesh tally (MESHTAL) output file and storing the values in a data structure. The mesh tally in MCNP calculates the reaction rate per source particle across the image plane of the NRAD beamline model. The mesh is composed of $450 \times 450$ voxels, simulating a $8 \times 8$ centimeter image plane. The simulation uses Equation 4.2 to calculate the activity of each voxel when the foil is mated to the film using the reaction rate data produced by MCNP:

$$
A=\left(1-e^{-\lambda t_{\text {shot }}}\right) \cdot S P R \cdot \operatorname{vol} \cdot e^{-\lambda t_{\text {travel }} \cdot R}
$$

The source particle rate used in Equation 4.2 is the bare foil derived source particle rate from Table 3.3. The simulation then uses the characteristic curve of the specified film to model the change in optical density due to the radiation from the foil. Equation 4.3 calculates the optical density change caused by the activity of each voxel for AGFA D3s.c. film mated to dysprosium foil based on the results in Figure 4.3.

$$
O D=\max \left\{\begin{array}{c}
3.768 \cdot A-0.1772 \\
0.2
\end{array}\right.
$$

The activity $(A)$ in Equation 4.3 has units of $\mu \mathrm{Ci} / \mathrm{cm}^{2}$. The characteristic curve based on the measured activity of a specific foil represents the contribution of both the beta and gamma radiation.

Scanning a calibrated optical density step wedge prior to scanning the film allows for the creation of a calibration curve relating optical density to pixel value. Figure 4.4 shows the calibration curve calculated for the scanner used in this experiment using a fifth order polynomial fit. 


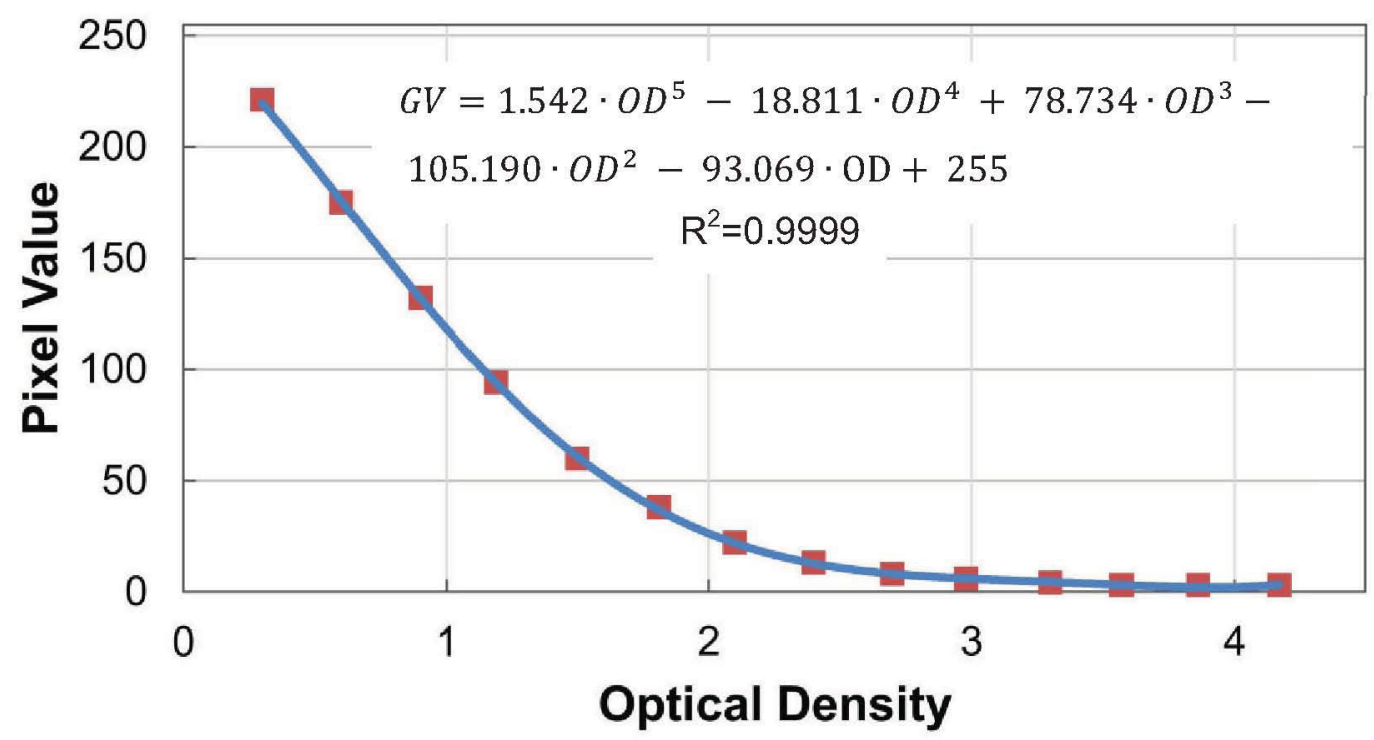

Figure 4.4. Calibration curve for a Fuji FineScan 1500 scanner and an Agfa DenStep calibrated optical density step wedge.

Equation 4.4, derived from the calibration curve in Figure 4.4, converts each optical density value into an 8-bit gray value:

$$
\begin{aligned}
& G V=1.542 \cdot O D^{5}-18.811 \cdot O D^{4}+78.734 \cdot O D^{3}-105.190 \cdot O D^{2}-93.069 \cdot \\
& O D+255
\end{aligned}
$$

The program writes the gray values to a file using the portable gray map (pgm) file format. This format is simple and readable by a variety of image analysis programs, including ImageJ.

\subsubsection{Image Simulation Validation}

An image of a polyethylene step block highlights the importance of film response in accurately simulating radiographs. Figure 4.5 provides the dimensions of the step block used to test the image simulation program. The different thicknesses of the block form areas of differing activity on the foil. This illustrates that the correlation between optical density and activity can assist in determining the thickness of a known material from a radiograph.

Figure 4.6 shows the scanned radiograph of the step block produced using the typical 


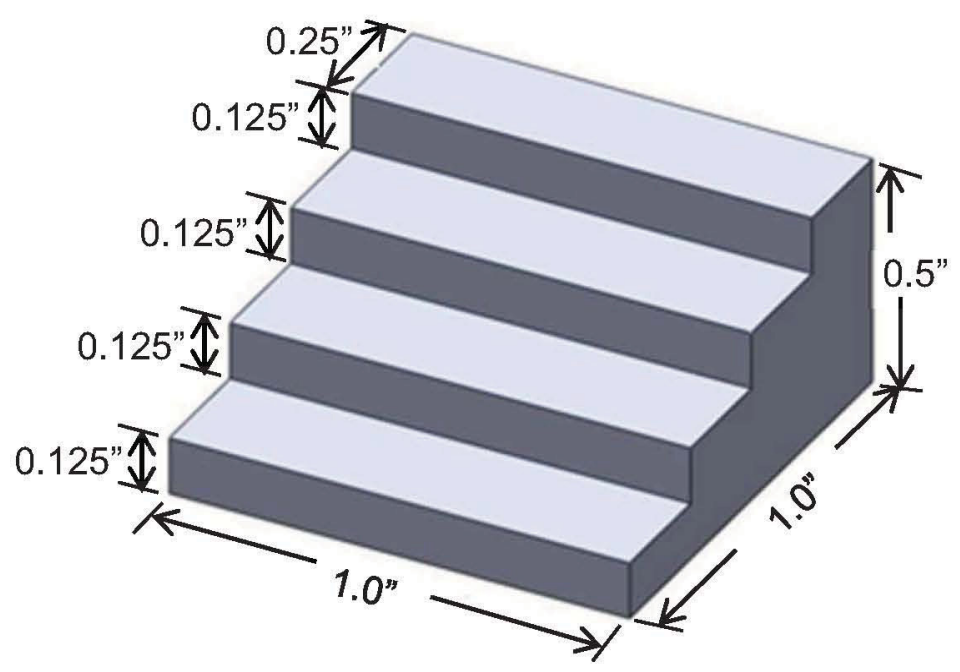

Figure 4.5. Polyethylene step block used to validate the image simulation program.

process at the NRAD. Figure 4.7.a displays the simulated image generated using the linear approximation in Equation 4.1. Figure 4.7.b represents the image generated using the film response and scanner calibration curves provided by Equations 4.3 and 4.4, respectively.

Tables 4.2 and 4.3 detail the average optical densities and pixel values, respectively, for the actual radiograph and the simulated images with and without the corrections for film and scanner response. Each image is divided into 12 regions, as shown in Figure 4.8. A densitometer measured the optical density in each region of the radiograph. The percent difference between the average values from each region of the actual radiograph and the averages from the corresponding regions of the simulated images are shown in parentheses in Tables 4.2 and 4.3. The simulated image is noticeably pixelated as a result of the high variances caused by the high resolution of the mesh. A lower resolution mesh run with more particles would result in lower tally uncertainty for each pixel, and a smoother image.

The optical density varies across each step, with a maximum optical density in the center of each step (see Figures 4.6 and 4.7.b and Table 4.2). The simulation does show this behavior, but it is more pronounced than in the actual radiograph. The predicted optical 


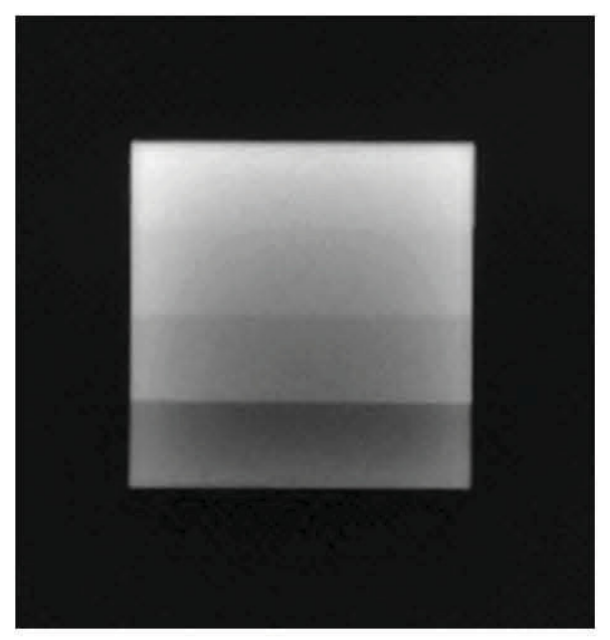

Figure 4.6. Scanned radiograph of the polyethylene step block shown in Figure 4.5.

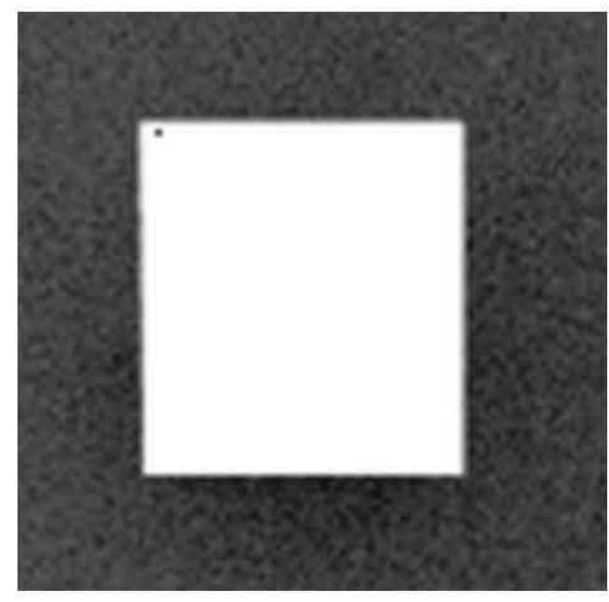

(a) uncorrected

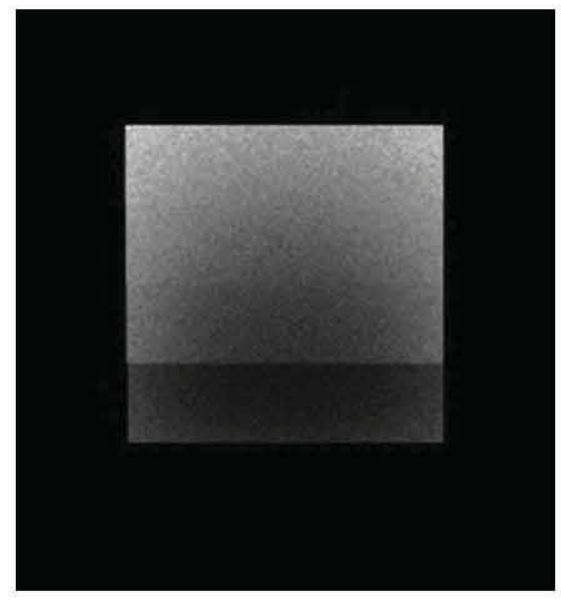

(b) corrected

Figure 4.7. Simulated radiographs of the polyethylene step block in Figures 4.5 and 4.6 with and without the corrections for the film characteristic and scanner calibration curves.

densities in the corrected image are consistently lower than the optical densities of the actual radiograph. The step averaged optical density calculated by the corrected simulation differs 
Table 4.2. Optical density values for the polyethylene step block in Figures 4.6 and 4.7.

\begin{tabular}{cccccc}
\hline & & Left & Center & Right & Average \\
\hline \multirow{2}{*}{ Step 1 } & Actual & 0.72 & 0.78 & 0.79 & 0.75 \\
& Predicted & $0.47(-34.7 \%)$ & $0.57(-26.9 \%)$ & $0.43(-45.5 \%)$ & $0.49(-34.7 \%)$ \\
\hline Step 2 & Actual & 0.98 & 1.17 & 1.09 & 1.08 \\
& Predicted & $0.77(-21.4 \%)$ & $0.95(-18.8 \%)$ & $0.72(-33.9 \%)$ & $0.83(-23.1 \%)$ \\
\hline Step 3 & Actual & 1.47 & 1.58 & 1.49 & 1.53 \\
& Predicted & $1.10(-25.2 \%)$ & $1.36(-13.9 \%)$ & $1.06(-28.8 \%)$ & $1.17(-23.5 \%)$ \\
\hline Step 4 & Actual & 1.87 & 1.91 & 1.84 & 1.89 \\
& Predicted & $1.64(-12.3 \%)$ & $1.83(-4.2 \%)$ & $1.56(-15.2 \%)$ & $1.67(-11.6 \%)$ \\
\hline
\end{tabular}

Table 4.3. Pixel values for the polyethylene step block in Figures 4.6 and 4.7.

\begin{tabular}{cccccc}
\hline & & Left & Center & Right & Average \\
\hline Step 1 & Actual & 175 & 159 & 169 & 170 \\
& Uncorrected & $247(+41.1 \%)$ & $245(+54.1 \%)$ & $247(+46.2 \%)$ & $247(+45.3 \%)$ \\
& Corrected & $188(+7.4 \%)$ & $175(+10.1 \%)$ & $192(+13.6 \%)$ & $183(+7.7 \%)$ \\
\hline Step 2 & Actual & 135 & 108 & 128 & 123 \\
& Uncorrected & $241(+78.5 \%)$ & $235(+117.6 \%)$ & $241(+88.3 \%)$ & $238(+93.5 \%)$ \\
& Corrected & $148(+9.6 \%)$ & $121(+12.0 \%)$ & $151(+18.0 \%)$ & $139(+13.0 \%)$ \\
\hline Step 3 & Actual & 99 & 70 & 91 & 86 \\
& Uncorrected & $231(+133.3 \%)$ & $224(+220 \%)$ & $231(+153.8 \%)$ & $229(+166.3 \%)$ \\
& Corrected & $97(-2.0 \%)$ & $69(-1.4 \%)$ & $101(+11.0 \%)$ & $89(+3.5 \%)$ \\
\hline Step 4 & Actual & 62 & 44 & 57 & 53 \\
& Uncorrected & $219(+253.2 \%)$ & $212(+381.8 \%)$ & $218(+282.5 \%)$ & $217(+309.4 \%)$ \\
& Corrected & $49(-21.0 \%)$ & $32(-27.3 \%)$ & $52(-8.8 \%)$ & $44(-17.0 \%)$ \\
\hline
\end{tabular}

from the actual image by $-11.6 \%$ for the fourth step (Table 4.2 ). This difference generally increases on each step moving up the block until the first step where the step averaged optical density of the corrected simulation differs from the actual image by $-34.7 \%$.

The step averaged pixel values of the uncorrected simulation differ from the step averaged pixel values of the actual radiograph by more than $45.3 \%$ on all steps. The step averaged pixel value from the first step of the corrected simulation differs from the step averaged pixel value of the actual radiograph by $+7.7 \%$. The difference between the step averaged pixel value for the corrected image and the actual radiograph is $-17.0 \%$ for the fourth step. 


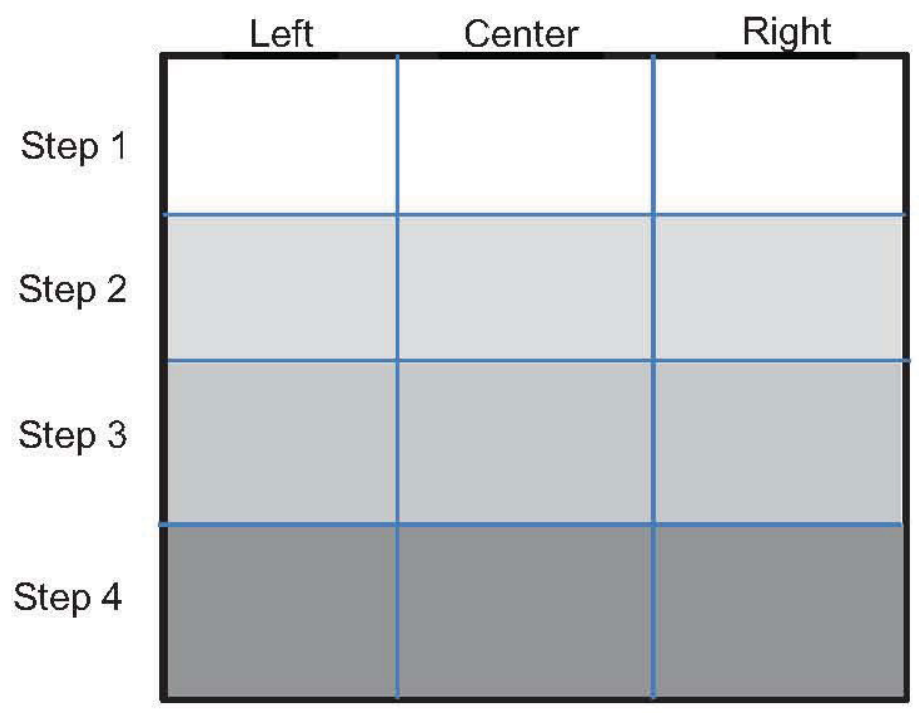

Figure 4.8. Averaging regions for the image data in Tables 4.2 and 4.3.

As a comparison, the step averaged pixel value for the fourth step of the uncorrected image differs by $+309.4 \%$ from the step averaged pixel value of the fourth step in the actual image and decreases to $+45.3 \%$ on the first step of the step block. In all cases, the corrected pixel values are significantly closer to the actual image pixel values.

The accuracy of the optical density data indicates that the characteristic curve in Figure 4.3 provides a reasonably accurate approximation for the response of the film to beta and gamma radiation. The difference between the actual and predicted optical densities increases as the optical density decreases. This may be due to inaccuracies in the characteristic curve at low optical densities. The characteristic curve in Figure 4.3 contains data points for optical density values above 2.0 , and the remainder of the curve is linearly extrapolated from this region. Based on Figures 2.5 and 2.6, this region most likely has a logarithmic behavior. Additional irradiations to fill in this part of the curve are recommended as part of future research.

\subsection{Summary of Results}

A film and scanner response correction program allows MCNP data to be converted into an accurate simulation of a radiographic image. Accurately modeling the film response required 
a new characteristic curve relating AGFA D3 s.c. film optical density to dysprosium foil activity. An image simulation program using this curve can simulate dysprosium radiographs produced at the NRAD.

The simulation validation compared a radiograph of a polyethylene step block to a simulated radiograph of the same step block. The simulation calculates optical densities which agree reasonably well with actual film optical densities. The corrected radiograph simulates the actual radiograph more accurately for the thinner steps, resulting in step averaged optical density differences between the actual and simulated images of $-11.6 \%$ for the thinnest step versus a difference of $-34.7 \%$ for the thickest step, possibly due to the greater accuracy of the higher optical density region of the characteristic curve.

Applying the scanner calibration curve to the calculated optical density values decreases the difference between the actual radiograph pixel values and the simulated pixel values for each step except the fourth step (corresponding to the thinnest step and the darkest region of the image). The step averaged differences between the corrected and actual images increase from $-11.6 \%$ to $-17.0 \%$ for the thinnest step and decrease from $-34.7 \%$ to $+7.7 \%$ for the thickest step after the calibration curve is applied.

The smoothness of the simulated image could be improved by lowering the resolution of the mesh and increasing the number of particles included in the simulation. Accurate correction curves will allow NRAD to produce simulated radiographs. This will allow NRAD to extract more useful information from their radiographs, such as material density, or thickness of well characterized materials. 


\section{CHAPTER 5}

\section{SUMMARY AND CONCLUSIONS}

A fully characterized neutron beam is important for producing reproducible and comparable results at neutron radiography facilities. Since all neutron beams are different, characterization allows different facilities to effectively compare radiographs. Characterization also allows facilities to more efficiently track changes in radiographic parameters over time, such as beam quality and effective collimation ratio.

The effective collimation ratio, beam quality, divergence, flux profile, and energy spectrum have been characterized at the NRAD. The results of this characterization show that the NRAD has an L/D greater than 125 , a beam divergence of $0.3 \pm 0.1$ degrees, and is a category I radiographic facility.

Activation of bare foils in the NRAD east neutron radiography beam measured the thermal flux in the beam. In 2011, 13 bare foils and 4 cadmium covered foils were irradiated in the east radiography station. In 2012, 17 cadmium covered foils were irradiated in the same location. The calculation of the per foil MCNP source particle rates based on the comparison of the measured and calculated fluxes and reaction rates for each foil provides an evaluation of the accuracy of the modeled neutron energy spectrum. The source particle rates calculated for the bare foils are consistent across all of the bare foils, and the source particle rates calculated for the cadmium covered foils are consistent across all of the cadmium covered foils; however, the bare and cadmium covered source particle rates differ by an order of magnitude. Experimental evidence suggests that the thermal region is over-sampled by the current energy spectrum used in the NRAD beamline model. The replacement of the NRAD's highly enriched core with lowenriched fuel increased the resonance capture by U-238, which is not accounted for in the current model spectrum. 
In 2012, the NRAD irradiated an array of 6 gold and 6 dysprosium foils at the east radiography station. The array of gold and dysprosium activation foils measured the variation in flux across the image plane. The calculated flux averaged over either the gold foils or dysprosium foils varied by less than $5.4 \%$ over the area of the image plane. The source particle rates calculated from these bare foil irradiations agree well with the source particle rates calculated from the 2011 bare foil irradiations. Based on the 2011 bare foil irradiations, the predicted total flux at the image plane of the NRAD is $5.54 \times 10^{6} \mathrm{n} / \mathrm{cm}^{2} \mathrm{~s}$. The flux data from the 2011 and 2012 irradiations indicate that the NRAD east beamline has a gold foil cadmium ratio of 2.7 or 2.9 , respectively.

More relevant information could be extracted from radiographs if the resulting images could be accurately simulated. Simulations could provide information like the material density or the thickness of an object with well characterized material properties. A film and scanner response correction program allows MCNP data to be converted into accurate image simulations. Creation of the program necessitated the creation of a new film characteristic curve relating foil activity to optical density for AGFA D3s.c. film mated to dysprosium foil irradiated in the NRAD.

The resulting image simulation method is tested with actual and simulated images of a polyethylene step block. The image generated using film and scanner response curves provides a much more accurate radiograph than the image generated using only the MCNP data. The simulated step averaged optical densities for a polyethylene step block differ from the step averaged optical densities measured on a radiograph of the step block by less than $35 \%$ for all steps. The corrected radiograph simulates the actual radiograph more accurately for the thinner steps; there is a step averaged $-11.6 \%$ difference in optical densities for the thinnest step, compared to a step averaged $-34.7 \%$ difference for the thickest step. When the scanner calibration curve is applied, the step averaged differences increase from $-11.6 \%$ to $-17.0 \%$ for the thinnest step and decrease from $-34.7 \%$ to $+7.7 \%$ for the thickest step. The smoothness of 
the simulated image could be improved using a lower resolution mesh and more source particles in the MCNP model. 


\section{CHAPTER 6}

\section{SUGGESTIONS FOR FUTURE RESEARCH}

Further characterization work could be completed, including measurement of the neutron to gamma ratio for the NRAD beamline. This could be accomplished using the Fricke Reference Dosimetry System (ASTM International, 2004), or by irradiating a thermoluminescent dosimeter or an optically stimulated luminescence dosimeter. Irradiating a non-neutron sensitive dosimeter on an empty film cassette would measure the gamma dose without measuring the neutron dose.

The NU device could not be moved far enough away from the image plane to precisely measure the L/D of the NRAD. Turning the film cassette 180 degrees creates a gap of 1 inch between the d-section wall and the foils. Taking an image of the NU device with this cassette configuration might allow the NU device to measure the absolute L/D. Modifying the NU device to contain smaller diameter wires would increase the range of measureable L/D values.

Using the existing benchmarked NRAD MCNP model (Bess et al., 2011) to derive a neutron energy spectrum for the beamline model may result in a more accurate neutron energy spectrum. A full spectrum unfolding using additional bare and cadmium covered activation foil data could also provide a more accurate neutron energy spectrum for the NRAD beamline model.

As a consequence of the difficulties in obtaining irradiation time at the NRAD, there are only four data points on the characteristic curve for the dysprosium foils. Generating more data points would make the curve more accurate and useful over a larger range of optical densities. A more complete characteristic curve would improve the accuracy of the image simulation program. Characteristic curves for indium foils and different radiography films should be generated using the procedures presented in this work. 
Additionally, the simulation could be made more accurate by adjusting the resolution and number of particles run to reduce uncertainty in the tally data. 


\section{REFERENCES CITED}

Aghara, S. and Charlton, W., "Characterization and Quantification of an In-Core Neutron Irradiation Facility at a TRIGA-II Research Reactor," Nuclear Instruments and Methods in Physics Research B, 248, 2006, pp. 181-190.

Arai, M., and Crawford, K., "Neutron Sources and Facilities," in Anderson, I.S., McGreevy, R.L., and Bilheux, H.Z., (Ed.) Neutron Imaging and Applications A Reference for the Imaging Community, Springer, New York, NY, 2010, pp. 13-30.

ASTM International, "Standard Test Method for Determining the L/D Ratio of Neutron Radiography Beams," ASTM Standard E803-91, 1991.

ASTM International, "Standard Practice for Thermal Neutron Radiography of Materials," ASTM Standard E748-02, 2002.

ASTM International, "Standard Practice for Using the Fricke Reference Standard Dosimetry System," ASTM Standard E1026-04, 2004.

ASTM International, "Standard Test Method for Determining Image Quality in Direct Thermal Neutron Radiographic Examination," ASTM Standard E545-05, 2005.

ASTM International, "Standard Guide for Selection and Use of Neutron Sensors for Determining Neutron Spectra Employed in Radiation-Hardness Testing of Electronics," ASTM Standard E720-08, 2008.

ASTM International, "Standard Practice for Determining Neutron Fluence, Fluence Rate, and Spectra by Radioactivation Techniques," ASTM Standard E261-10, $2010 \mathrm{a}$.

ASTM International, "Standard Practice for Fabrication of the Neutron Radiographic Sensitivity Indicator," ASTM Standard E2023-10, 2010c.

ASTM International, "Standard Practice for Fabrication of the Neutron Radiographic Beam Purity Indicators," ASTM Standard E2003-10, 2010b.

Auterinen, I., Seren, T., Anttila, K., Kosunen, A., and Savolainen, S., "Measurement of Free Beam Neutron Spectra at Eight BNCT Facilities Worldwide," Applied Radiation and Isotopes, 61, 2004, pp. 1021-1026.

Berger, H. and Iddings, F., "Neutron Radiography," Nondestructive Testing Information Analysis Center Report, NTIAC-SR-98-01, 1998.

Bess, J.D., Maddock, T.L., Marshall, M.A., and Montierth, L.M., "Fresh-Core Reload of the Neutron Radiography (NRAD) Reactor with Uranium(20)-Erbium-Zirconium-Hydride Fuel," Idaho National Laboratory Report EXT-10-19486, March, 2011.

Carestream Health Inc. "Kodak Industrex T-200 Film TI-2360," Rochester, NY, February, 2008. 
Chadwick, M.B., Obložinský, P., Herman, M., Greene, N.M., Mcknight, R.D., Smith, D.L., Young, P.G., "ENDF/B-VII.0: Next Generation Evaluated Nuclear Data Library for Nuclear Science and Technology," Nuclear Data Sheets, 107, 2006, pp. 367-600.

Craft, A.E., and King, J.C., "Development of a Neutron Radiography and Tomography Facility at the Colorado School of Mines," Transactions of the American Nuclear Society, vol. 104, 2011, pp. 225-226.

Crow, L., "Neutron Detectors for Imaging," in Anderson, I.S., McGreevy, R.L., and Bilheux, H.Z., (Ed.) Neutron Imaging and Applications: A Reference for the Imaging Community, Springer, New York, NY, 2010, pp. 47-63.

Davidson, D.L., Kahn, S., "Radiography and Foil Handling," NRAD-OI-5530 Idaho National Laboratory, 2006.

de Almeida, G.L., Silvani, M.I., Furieri, C.A.A., "Evaluation of the Divergence of a Thermal Neutron Beam Using a Position Sensitive Detector," Brazilian Journal of Physics, 35, 3B, 2005, pp. 771-774.

Döler, W., Rassow, St., Jäger, A., and Vosshenrich, R., "Investigation of the Imaging Properties of an X-ray Film Scanner," Physics in Medicine and Biology, 39, 1994, pp. 917-922.

GE Sensing \& Inspection Technologies, "X-Ray Radiographic Film Systems," GEIT-40007EN, 2008 , pp. 13.

Gonzalez-Lopez, A., "Useful Optical Density Range In Film Dosimetry: Limitations Due To Noise And Saturation," Physics In Medicine And Biology, 52, 2007, pp. 321-327.

Heller, A.K., and Brenizer, J.S., "Neutron Radiography," in Anderson, I.S., McGreevy, R.L., and Bilheux, H.Z., (Ed.) Neutron Imaging and Applications: A Reference for the Imaging Community, Springer, New York, NY, 2010, pp. 67-80.

Hendee, W. H., Ritenour, E.R., Medical Imaging Physics, John Wiley \& Sons, New York, NY 2002, pp. 96.

Herwig, K.W., "Introduction to the Neutron," in Anderson, I.S., McGreevy, R.L., and Bilheux, H.Z., (Ed.) Neutron Imaqing and Applications: A Reference for the Imaging Community, Springer, New York, NY, 2010, pp. 3-12.

Howerton, R.J., Perkins, S.T., Haight, R.C., and MacGreggor, M.H., "Ta-181 Evaluated Neutron Cross Sections," ENDF/B-VII Incident Neutron Data, Lawrence Livermore Laboratory, 2006.

Imel, G.R. and Urbatsch, T., "Beam Characterization at the Neutron Radiography Facility (NRAD)," World Conference on Neutron Radiography, San Francisco, CA, May 10, 1992, pp. 55-60.

Kirk, M.A. and Greenwood, L.R., "Determination of the Neutron Flux and Energy Spectrum in the Low-Temperature Fast-Neutron Facility in CP-5, Calculations of Primary-Recoil and Damage-Energy Distributions, and Comparisons with Experiment," Journal of Nuclear Materials, 80, 1979, pp. 159-171. 
Kobayashi, H., "Beam Formation and Characterization for Neutron Radiography," Nondestructive Testing and Evaluation, 16, 2001, pp. 121-129.

Malkawi, S.R. and Ahmad, N., "Prediction and Measurement of Neutron Energy Spectrum in a Material Test Research Reactor," Annals of Nuclear Energy, 27, 2000, pp. 311-327.

McNaught, A.D. and Wilkinson, A., Compendium of Chemical Terminology, Blackwell Science, Malden, MA, 1997, pp. 203.

Nemec, T., Rant, J.J., Kristof, E., and Glumac, B., "Characterization of the Ljubljana TRIGA Thermal Column Neutron Radiographic Facility," Second Regional Meeting of the Nuclear Society of Slovenia, Portoroz, Slovenia, 1995, pp. 161-167.

Pope, C.L., "Spent Nuclear Fuel Assembly Inspection Using Neutron Computed Tomography", Ph.D. Dissertation, Idaho State University, 2010.

Pritychenko, B., Sonzogni, A.A., Winchell, D.F., Zerkin, V.V., Arcilla, R., Burrows, T.W., Dunford, C.L., Herman, M.W., McLane, V., Oblozinsky, P., Sanborn, Y., Tuli, J.K., "Nuclear Reaction and Structure Data Services of the National Nuclear Data Center," Annals of Nuclear Energy, 33, 2006, pp. 390-399.

Quinn, R.A. and Sigl, C.C., Radiography in Modern Industry, Eastman Kodak Company, Rochester, NY, 1980, pp. 83.

Raj, B., Venkataraman, B., Practical Radiography, Alpha Science Int'I Ltd., 2004, p. 68.

Stephens, W.E., "HFEF/Neutron Radiography Reactor, System Design Description," Argonne National Laboratory - West, Doc. No. W0170-0004-SA, June, 1978.

Thornton, J., "Enhanced Radiography for Aircraft Materials and Components," Engineering Failure Analysis, 11, 2004, pp. 207-220.

X-5 Monte Carlo Team, "MCNP5 Manual Volume I: Overview and Theory," LA-UR-03-1987 Los Alamos National Laboratory, 2003.

Yoshii, K., and Kobayashi, H., "Characterization of the YAYOI Fast Neutron Radiography Field," Nuclear Instruments and Methods in Physics Research A, 377, 1996, pp. 68-71. 
APPENDIX A

NRAD BEAMLINE MCNP MODEL 


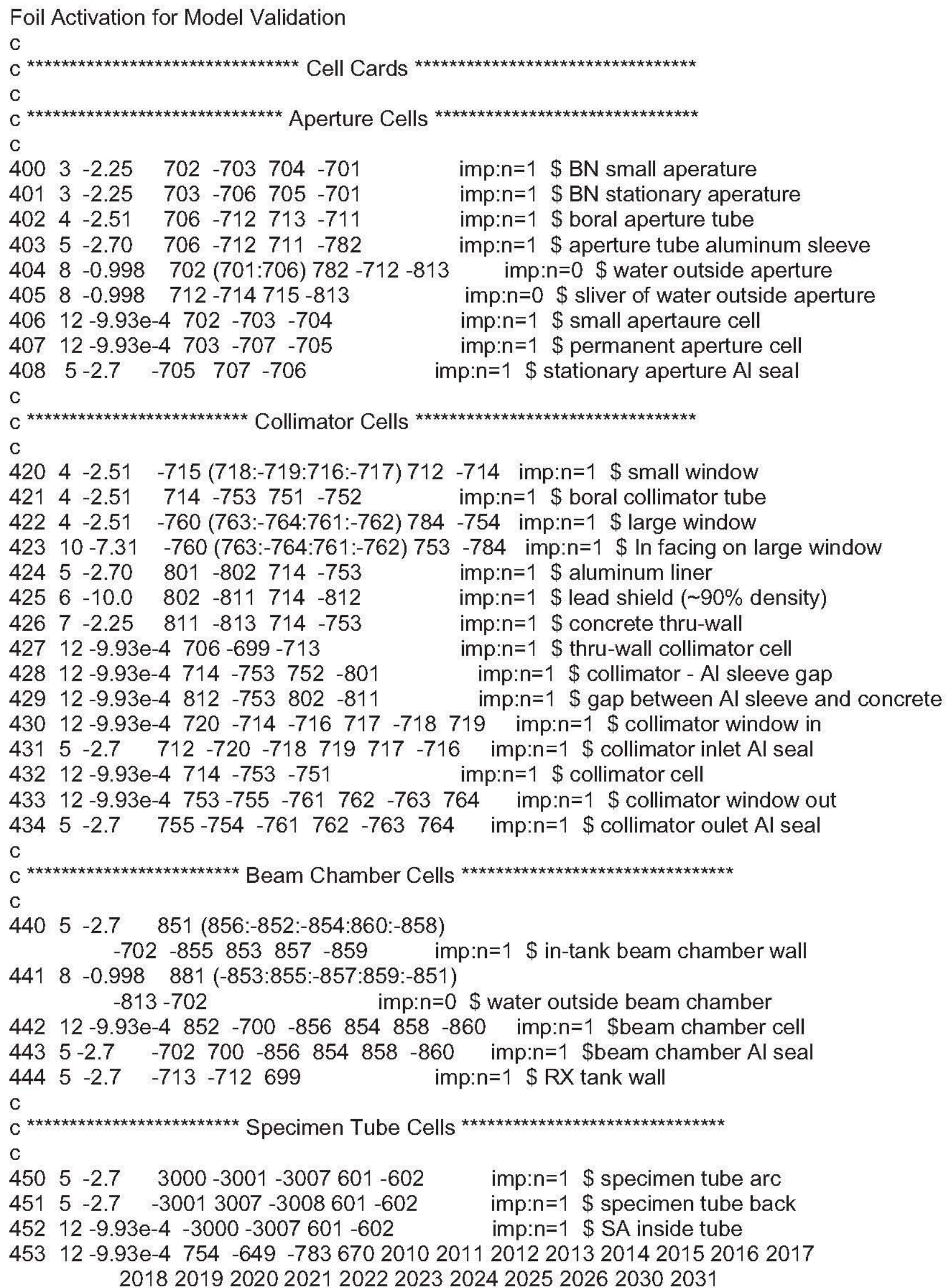


20322033203420352036203720382039204020412042

20432044204520462047204820492050205120522053

2054205520562057

(3001:3008:-601:602) imp:n=1 \$ beam cell

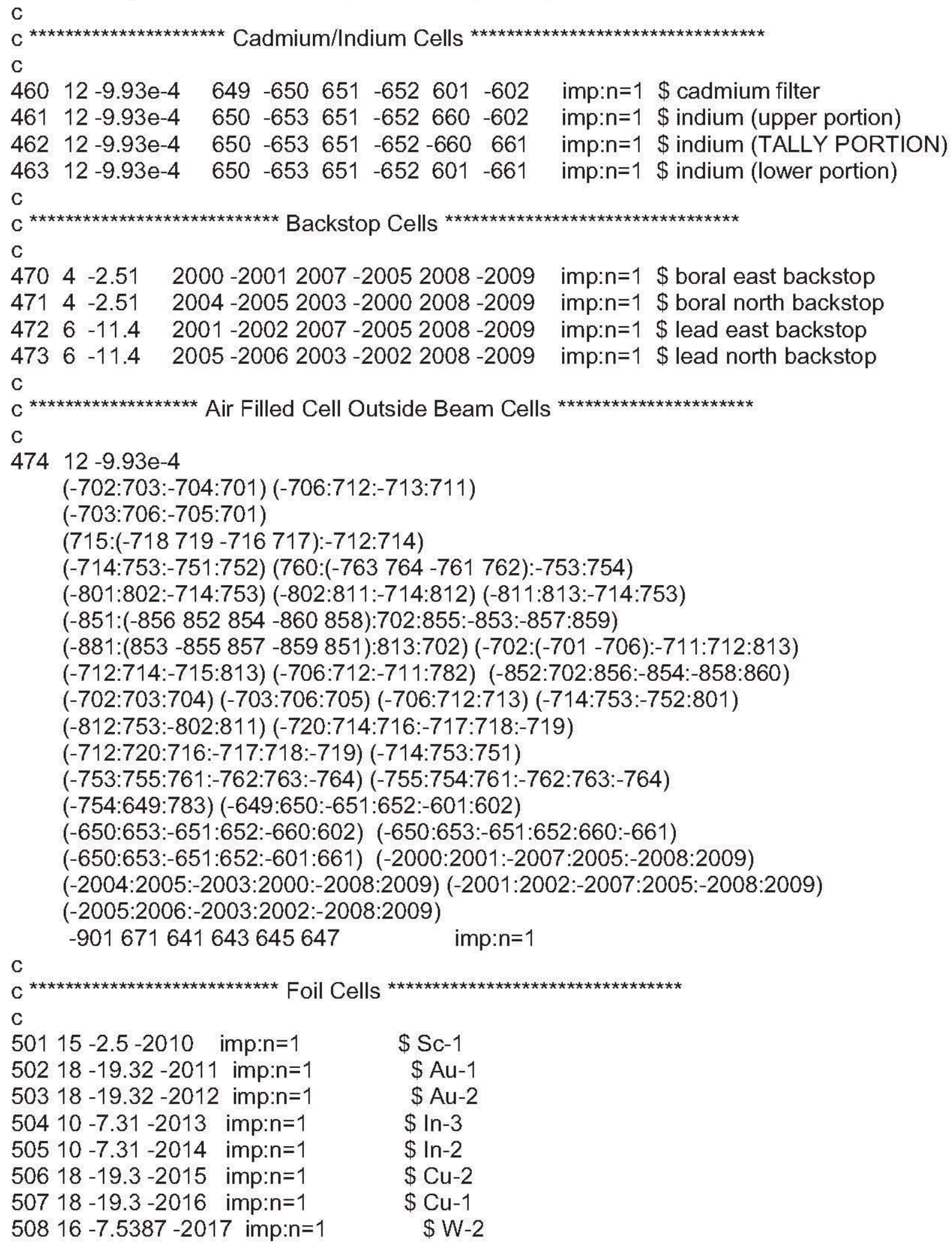


$50916-7.5387-2018$ imp:n=1

$51019-19.25-2019$ imp:n=1

$51119-19.25-2020$ imp:n=1

$51214-8.54-2021$ imp:n=1

$51314-8.54-2022$ imp: $n=1$

$51410-7.31-2023$ imp:n=1

$51510-7.31-2024$ imp: $n=1$

$51617-8.94-2025$ imp:n=1

$51717-8.94-2026$ imp:n=1

c

$5209-8.65-2030 \quad$ imp: $n=1$

$5219-8.65-2031$ imp:n=1

$5229-8.65-2032$ imp: $n=1$

$5239-8.65-2033$ imp: $n=1$

$5249-8.65-2034$ imp:n=1

$5259-8.65-2035$ imp: $n=1$

$5269-8.65-2036$ imp:n=1

$5279-8.65-2037$ imp:n=1

$5289-8.65-2038$ imp: $n=1$

$5299-8.65-2039$ imp:n=1

$5309-8.65-2040$ imp: $n=1$

$5319-8.65-2041 \quad$ imp: $n=1$

$5329-8.65-2042$ imp: $n=1$

$5339-8.65-2043$ imp: $n=1$

$5349-8.65-2044$ imp:n=1

$5359-8.65-2045$ imp: $n=1$

$5369-8.65-2046 \quad$ imp: $n=1$

$5379-8.65-2047$ imp: $n=1$

$5389-8.65-2048$ imp:n=1

$5399-8.65-2049$ imp:n=1

$5409-8.65-2050$ imp:n=1

$5419-8.65-2051 \quad$ imp: $n=1$

$5429-8.65-2052$ imp:n=1

$5439-8.65-2053$ imp:n=1

$5449-8.65-2054$ imp:n=1

$5459-8.65-2055$ imp: $n=1$

$5469-8.65-2056$ imp:n=1

$5479-8.65-2057$ imp:n=1
\$ W-1

$\$ \mathrm{MnCu}-2$

$\$ \mathrm{MnCu}-3$

$\$ \mathrm{Nb}-1$

$\$ \mathrm{Nb}-2$

$\$ \mathrm{Fe}-1$

\$ Ta-2

\$ Ta-1

$\$ \mathrm{Co}-1$

\$ Sc-1 front Cad cover \$ Sc-1 back Cad cover \$ Au-1 front Cad cover \$ Au-1 back Cad cover \$ Au-2 front Cad cover \$ Au-2 back Cad cover $\$$ In-3 front Cad cover $\$$ In-3 back Cad cover $\$$ In-2 front Cad cover $\$$ In-2 back Cad cover $\$ \mathrm{Cu}-2$ front Cad cover $\$$ Cu-2 back Cad cover $\$ \mathrm{Cu}-1$ front Cad cover $\$ \mathrm{Cu}-1$ back Cad cover $\$ W$-2 front Cad cover $\$ W-2$ back Cad cover $\$ W-1$ front Cad cover $\$ \mathrm{~W}-1$ back Cad cover $\$ \mathrm{MnCu}-2$ front Cad cover $\$ \mathrm{MnCu}-2$ back Cad cover $\$ \mathrm{MnCu}-3$ front Cad cover $\$ \mathrm{MnCu}-3$ back Cad cover $\$ \mathrm{Nb}-1$ front Cad cover $\$ \mathrm{Nb}-2$ front Cad cover $\$ \mathrm{Fe}-1$ front Cad cover \$ Ta-2 front Cad cover \$ Ta-1 front Cad cover $\$$ Co-1 front Cad cover

$\mathrm{c}$

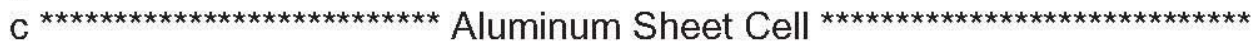

$\mathrm{C}$

$5605-2.7-670$ imp:n=1 \$ Front sheet $5615-2.7-671$ imp:n=1 \$ Back sheet

c

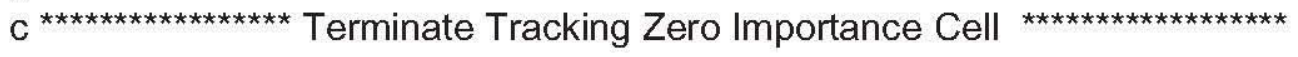

C

$4750901 \quad$ imp:n=0

C

C

$\mathrm{C}$ 


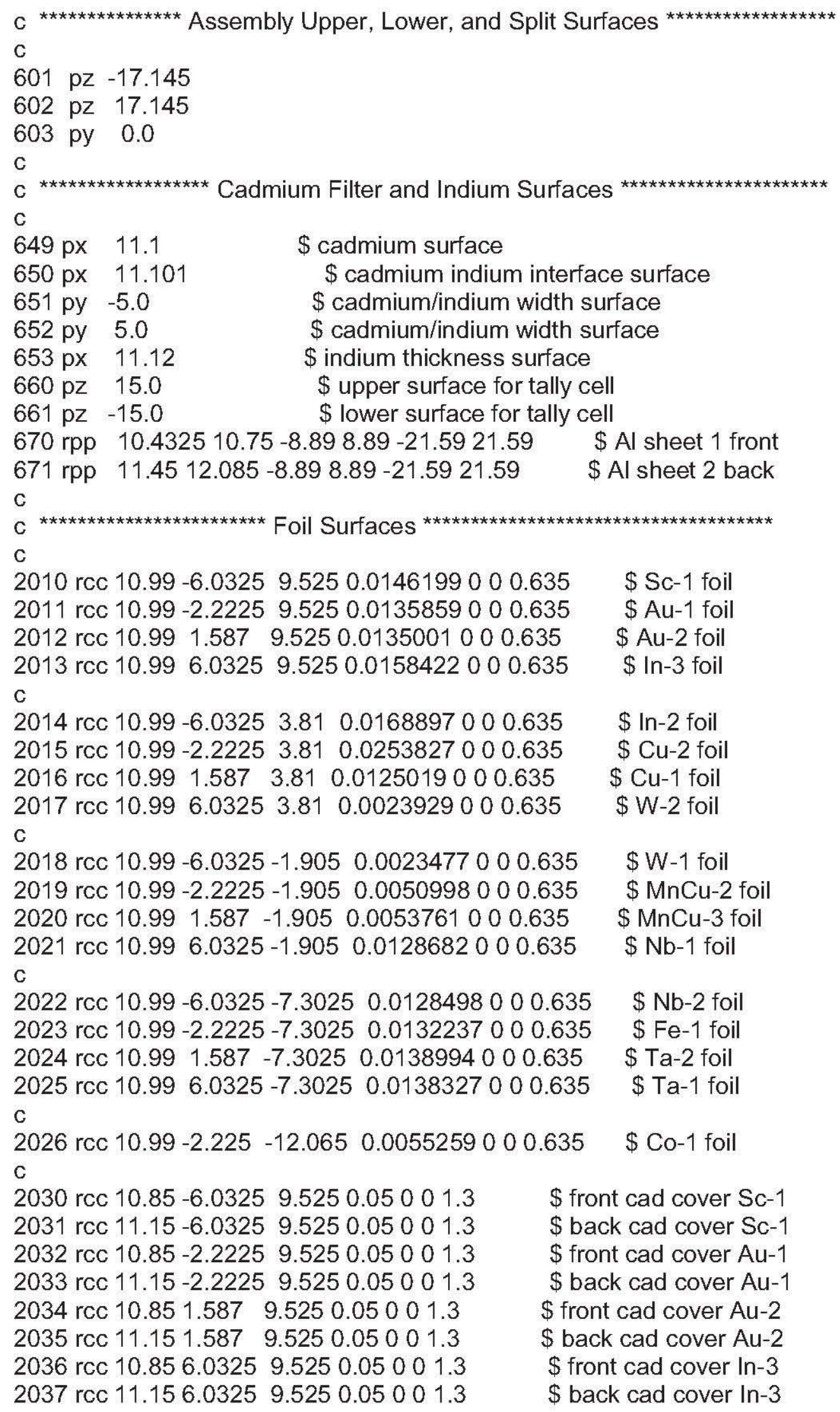


2038 rcc $10.85-6.03253 .810 .05001 .3$ 2039 rcc $11.15-6.03253 .810 .05001 .3$ 2040 rcc $10.85-2.22253 .810 .05001 .3$ 2041 rcc $11.15-2.22253 .810 .05001 .3$ $2042 \operatorname{rcc} 10.851 .587 \quad 3.810 .05001 .3$ 2043 rcc $11.151 .587 \quad 3.810 .05001 .3$ 2044 rcc 10.856 .03253 .810 .05001 .3 2045 rcc 11.156 .03253 .810 .05001 .3 C 2046 rcc $10.85-6.0325-1.9050 .05001 .3$ 2047 rcc $11.15-6.0325-1.9050 .05001 .3$ 2048 rcc $10.85-2.2225-1.9050 .05001 .3$ 2049 rcc $11.15-2.2225-1.9050 .05001 .3$ 2050 rcc $10.851 .587-1.9050 .05001 .3$ 2051 rcc $11.151 .587-1.9050 .05001 .3$

c

2052 rcc $10.856 .0325-1.9050 .05001 .3$ 2053 rcc $10.85-6.0325-7.30250 .05001 .3$ 2054 rcc $10.85-2.2225-7.30250 .05001 .3$ 2055 rcc $10.851 .587-7.30250 .05001 .3$ 2056 rcc $10.856 .0325-7.30250 .05001 .3$ 2057 rcc $10.85-2.225-12.0650 .05001 .3$ C

$\mathrm{C}$ $\mathrm{c}$

\begin{tabular}{|c|c|c|}
\hline 699 px & -254.6350 & \$ RX tank wall \\
\hline $700 \mathrm{px}$ & -289.8775 & $\$$ beam chamber Al seal \\
\hline $701 \mathrm{cx}$ & 12.7 & $\$$ aperture outside radius \\
\hline $702 \mathrm{px}$ & -289.56 & \$ small aperture surface near core \\
\hline $703 \mathrm{px}$ & -284.48 & $\$$ small aperture / stationary aperature interface \\
\hline $704 \mathrm{cx}$ & 1.7717 & $\$$ small aperture inside radius (hole) \\
\hline $705 \mathrm{cx}$ & 4.4450 & \$ stationary aperture inside radius (hole) \\
\hline 706 px & -279.40 & \$ stationary aperture / aperture tube interface \\
\hline 707 px & -279.5575 & \$ stationary aperture $\mathrm{Al}$ seal \\
\hline $711 \mathrm{cx}$ & 10.0013 & $\$$ aperture tube outside radius \\
\hline $712 \mathrm{px}$ & -254.00 & $\$$ aperture tube / collimator small window interface \\
\hline $713 \mathrm{cx}$ & 9.3282 & $\$$ aperture tube inside radius \\
\hline \\
\hline \\
\hline \\
\hline 714 px & -253.332 & \$ collimator small window / collimator tube interface \\
\hline $715 \mathrm{cx}$ & 28.5369 & $\$$ collimator small window end plate outside radius \\
\hline 716 py & 6.7640 & $\$$ collimator small window side \\
\hline 717 py & -6.7640 & \$ collimator small window side \\
\hline $718 \mathrm{pz}$ & 8.1102 & $\$$ collimator small window top \\
\hline $719 \mathrm{pz}$ & -8.1102 & $\$$ collimator small window bottom \\
\hline $720 \mathrm{px}$ & -253.84 & $\$$ collimator inlet seal surface \\
\hline $751 \mathrm{cx}$ & 17.780 & $\$$ collimator tube inside radius \\
\hline $752 \mathrm{cx}$ & 18.448 & $\$$ collimator tube outside radius \\
\hline $753 \mathrm{px}$ & -112.588 & $\$$ collimator tube / large window interface \\
\hline $754 \mathrm{px}$ & -111.920 & \$ collimator large window plate \\
\hline
\end{tabular}

$\$$ front cad cover In-2 $\$$ back cad cover In-2 $\$$ front cad cover $\mathrm{Cu}-2$ $\$$ back cad cover Cu-2 $\$$ front cad cover Cu-1 $\$$ back cad cover $\mathrm{Cu}-1$ $\$$ front cad cover W-2 $\$$ back cad cover $\mathrm{W}-2$

$\$$ front cad cover $\mathrm{W}-1$ $\$$ back cad cover $\mathrm{W}-1$ $\$$ front cad cover MnCu-2 $\$$ back cad cover $\mathrm{MnCu}-2$ $\$$ front cad cover $\mathrm{MnCu}-3$ $\$$ back cad cover $\mathrm{MnCu}-3$

$\$$ front cad cover $\mathrm{Nb}-1$ $\$$ front cad cover $\mathrm{Nb}-2$ $\$$ front cad cover Fe-1 $\$$ front cad cover Ta-2 $\$$ front cad cover Ta-1 $\$$ front cad cover Co-1

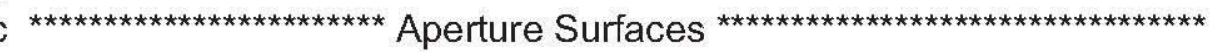




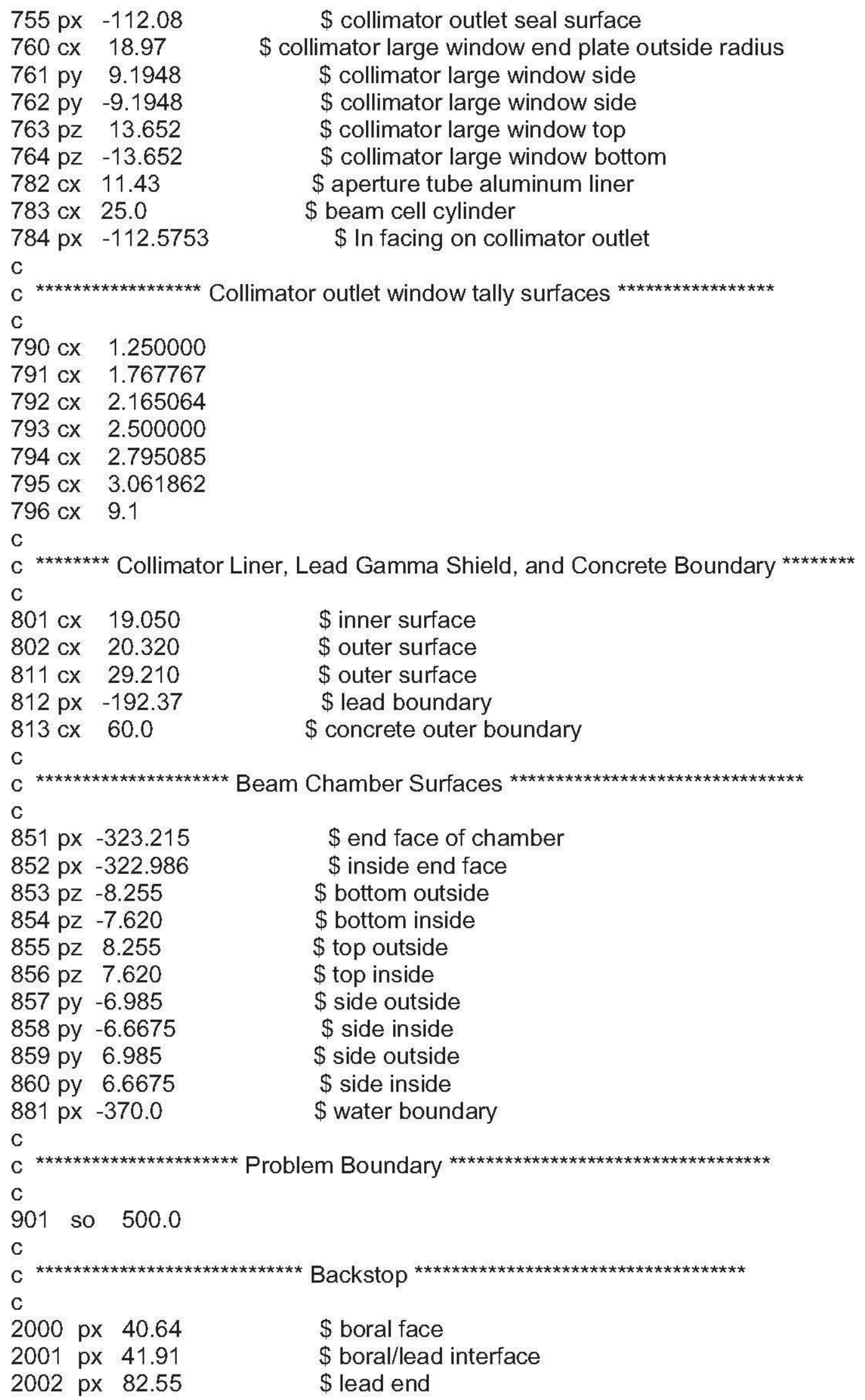




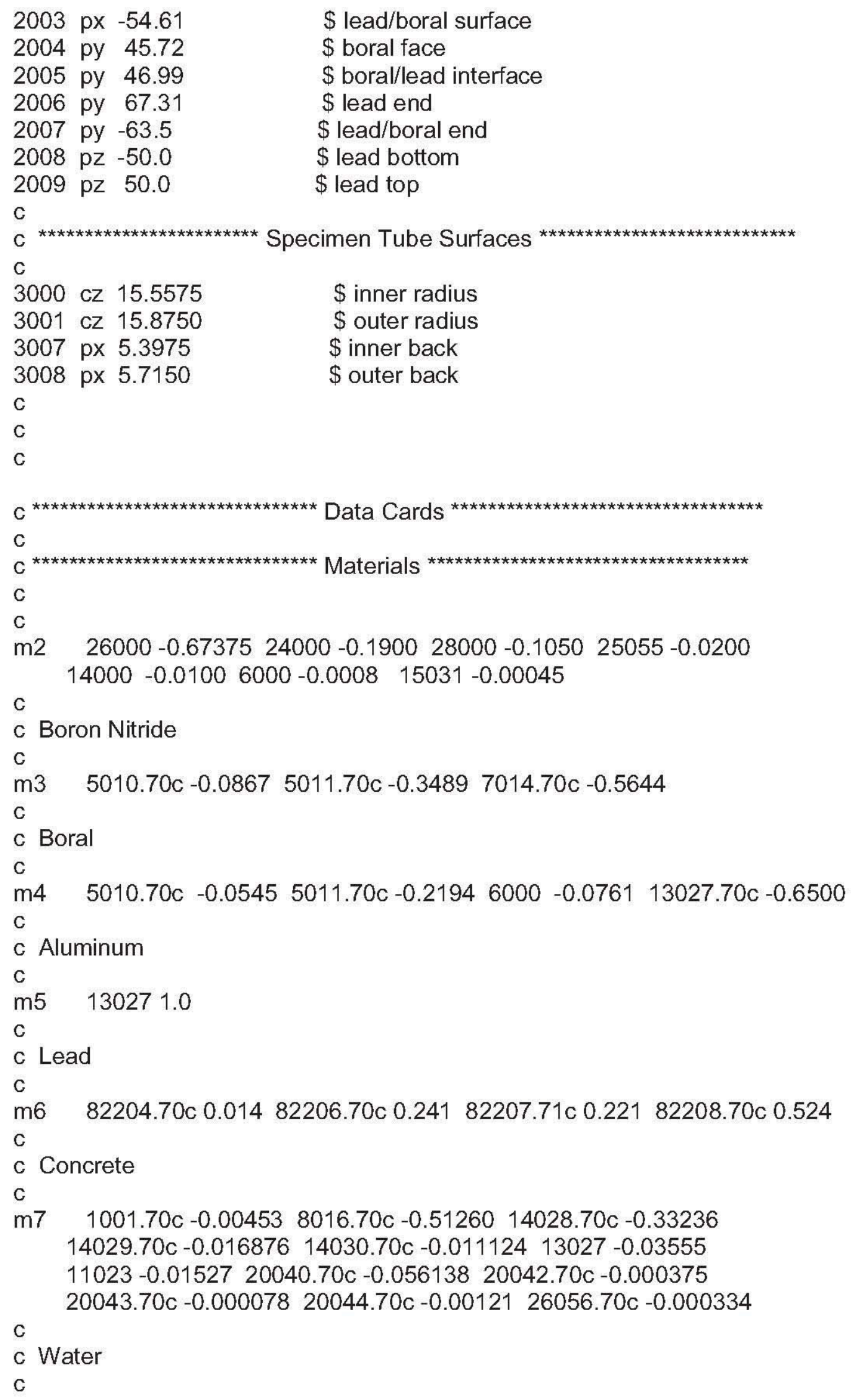




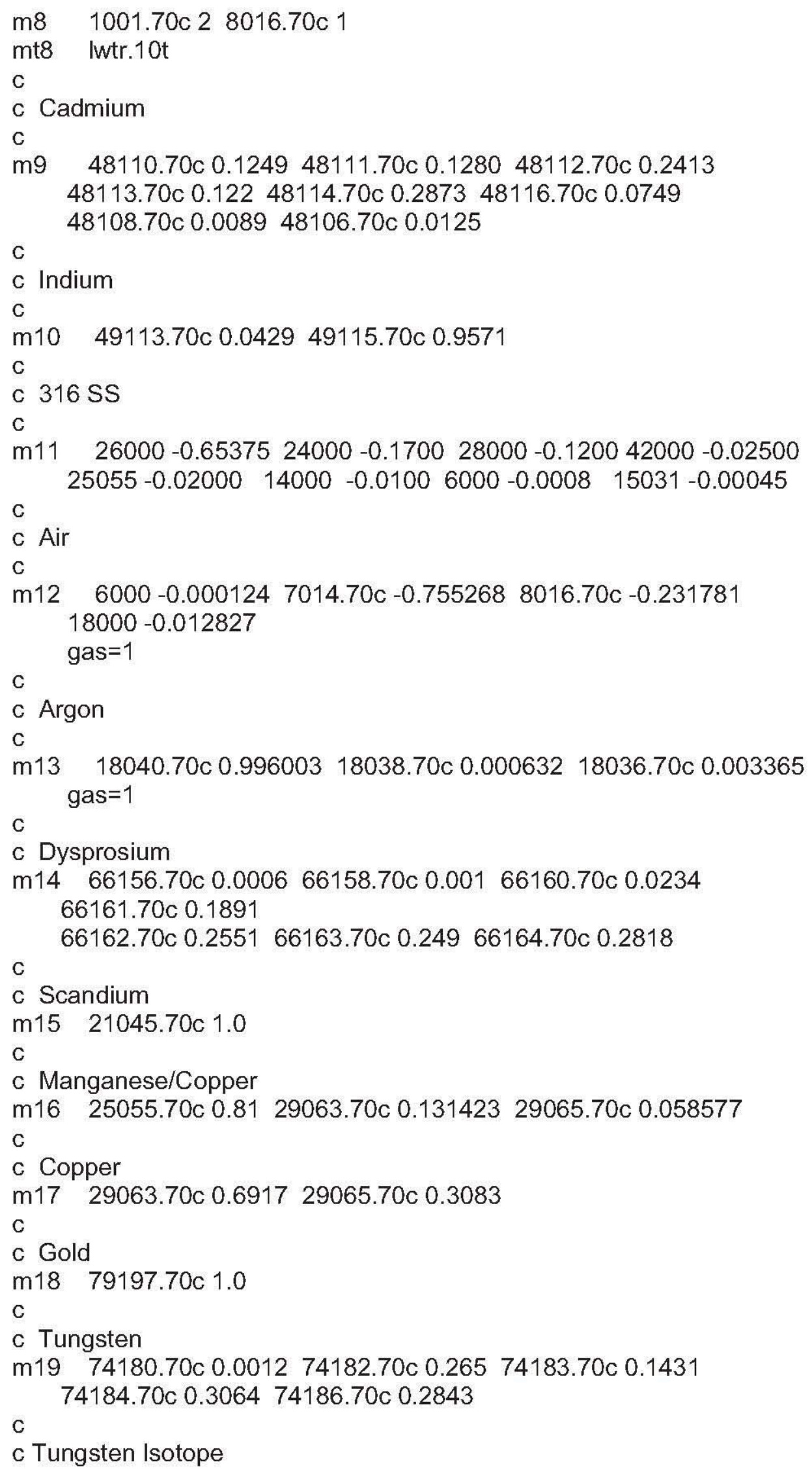




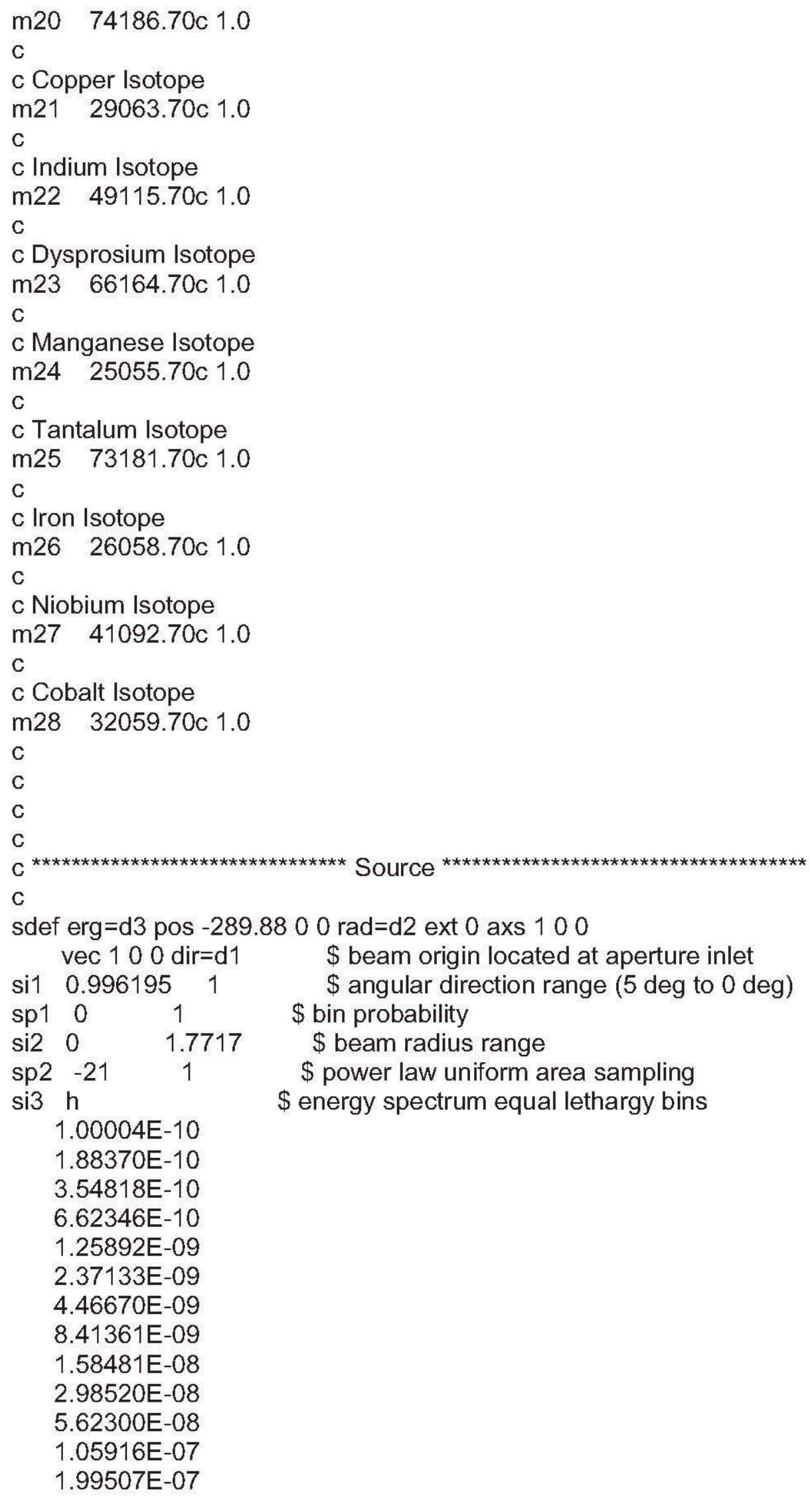




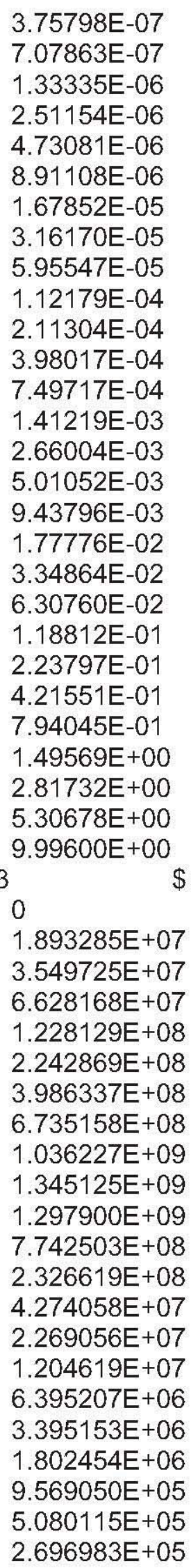




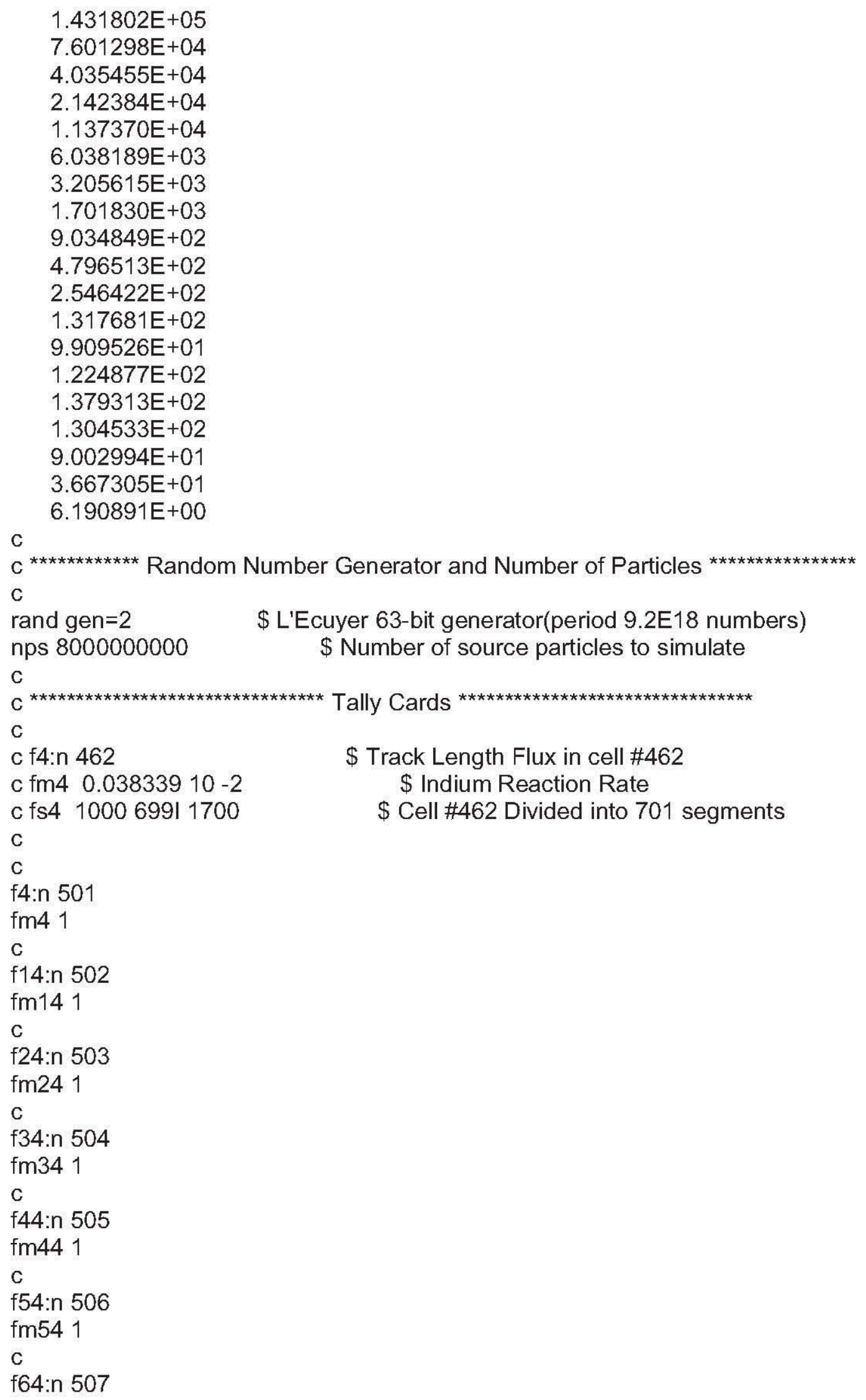


fm64 1

c

f74:n 508

fm74 1

c

f84:n 509

fm84 1

$\mathrm{c}$

f94:n 510

fm94 1

c

f104:n 511

fm104 1

c

f114: 512

fm114 1

c

f124:n 513

fm124 1

c

f134:n 514

fm134 1

C

f144:n 515

fm144 1

c

f154:n 516

fm154 1

c

f164:n 517

fm164 1

$\mathrm{c}$

c

C

f204:n 501

fm204 $0.03348815-2$

c

f214: 502

fm214 0.0590686 18 -2

c

f224: 503

fm224 0.059068618 -2

c

f234:n 504

fm234 $0.03669522-2$

C

f244: 505

fm244 $0.03669522-2$

C

f254:n 506

fm254 0.0587155 $21-2$ 
c

f264: 507

fm264 $0.058715521-2$

c

f274: 508

fm274 $0.017880520-2$

c

f284:n 509

fm284 0.0178805 20 -2

$\mathrm{c}$

f294:n 510

fm294 0.065969324 -2

C

f304:n 511

fm304 $0.065969324-2$

c

f314: 512

fm314 $0.055549227-2$

c

f324: 513

fm324 $0.055549227-2$

$\mathrm{c}$

f334:n 514

fm334 $0.000239426-2$

c

f344:n 515

fm3440.0196930 $25-2$

c

f354:n 516

fm354 0.0196930 $25-2$

c

f364: 517

fm364 $0.090943328-2$

$\mathrm{c}$

c

C

FMESH604:n GEOM=rec ORIGIN=7.95 -31.0 \$ Dy rxn fmesh IMESH=7.96016 IINTS=1

$\mathrm{JMESH}=5 \mathrm{JINTS}=450$

$\mathrm{KMESH}=9 \mathrm{KINTS}=450$

c $\quad E M E S H=0.5 e-60.110 .0$

fm604 0.00896914102

c

FMESH704:n GEOM=rec ORIGIN=8.30-31.0 \$In rxn fmesh IMESH $=8.31016$ IINTS $=1$

$\mathrm{JMESH}=5$ JINTS $=450$

$\mathrm{KMESH}=9 \mathrm{KINTS}=450$

c $\quad E M E S H=0.5 e-60.110 .0$

fm7040.0383410102

$\mathrm{C}$

C 


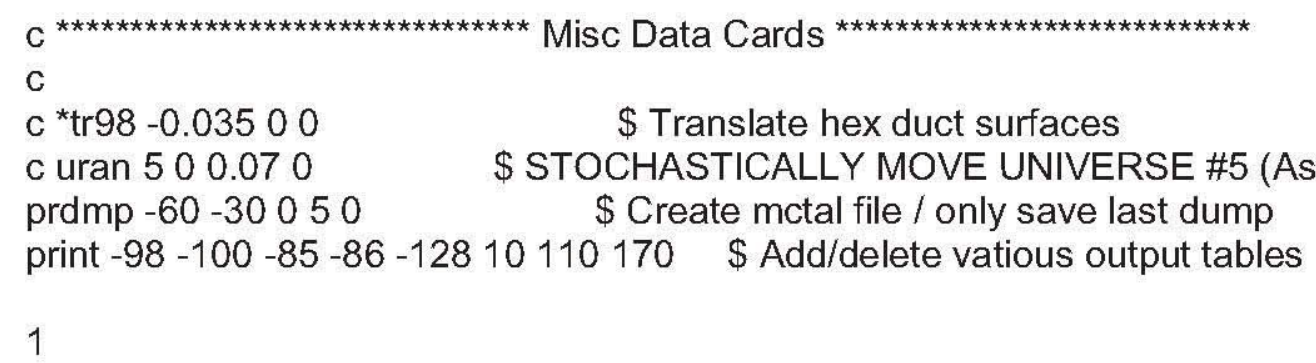


APPENDIX B

IMAGE SIMULATION PROGRAM 


\section{RadiographSimulation.cpp}

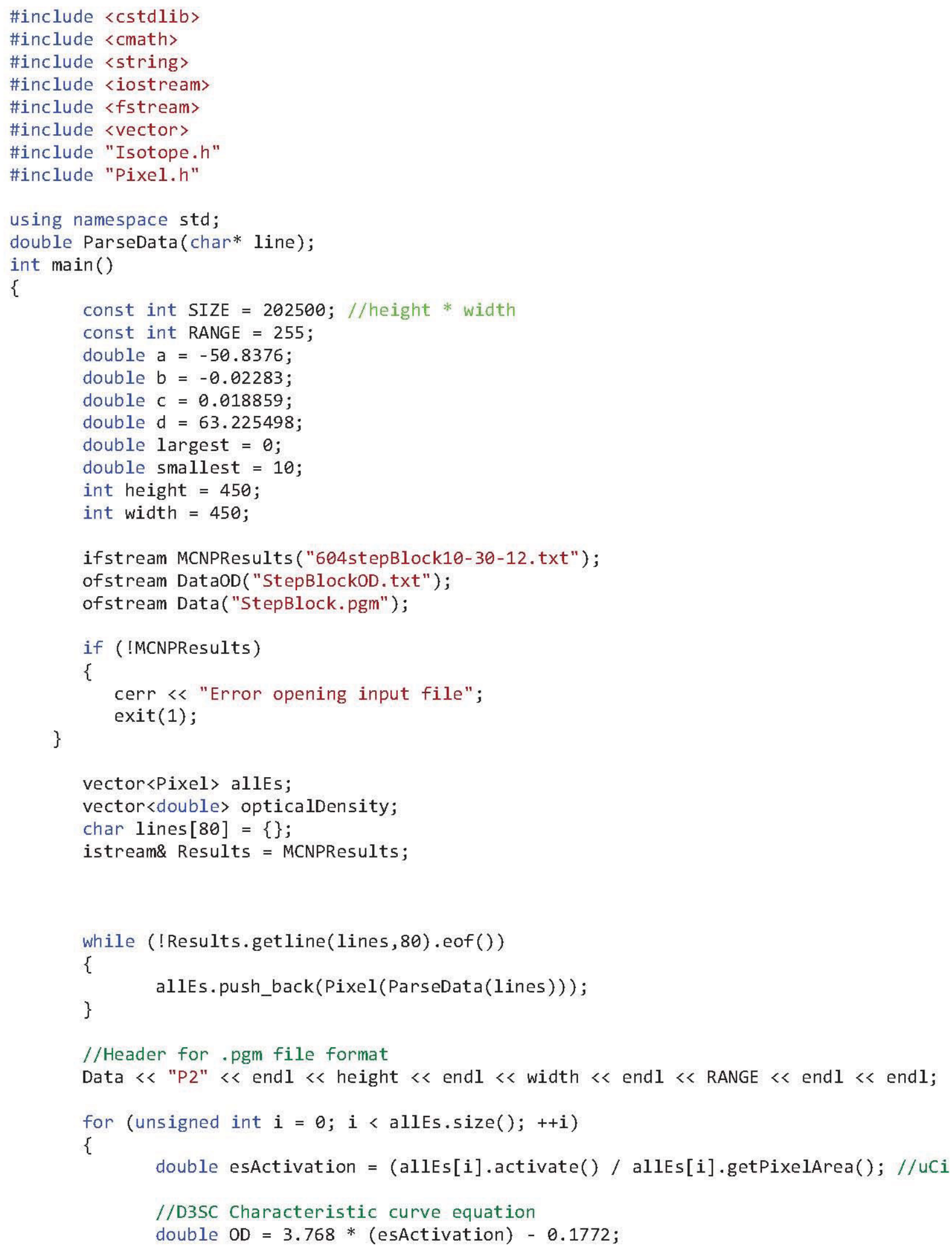




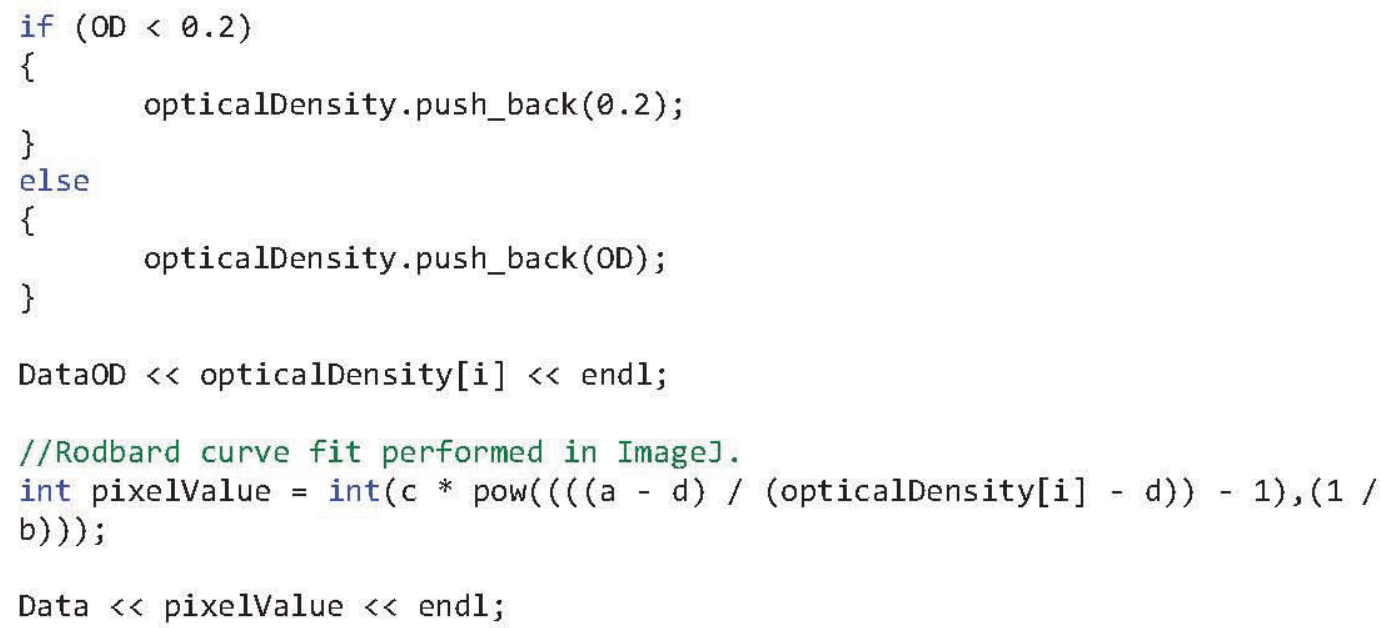




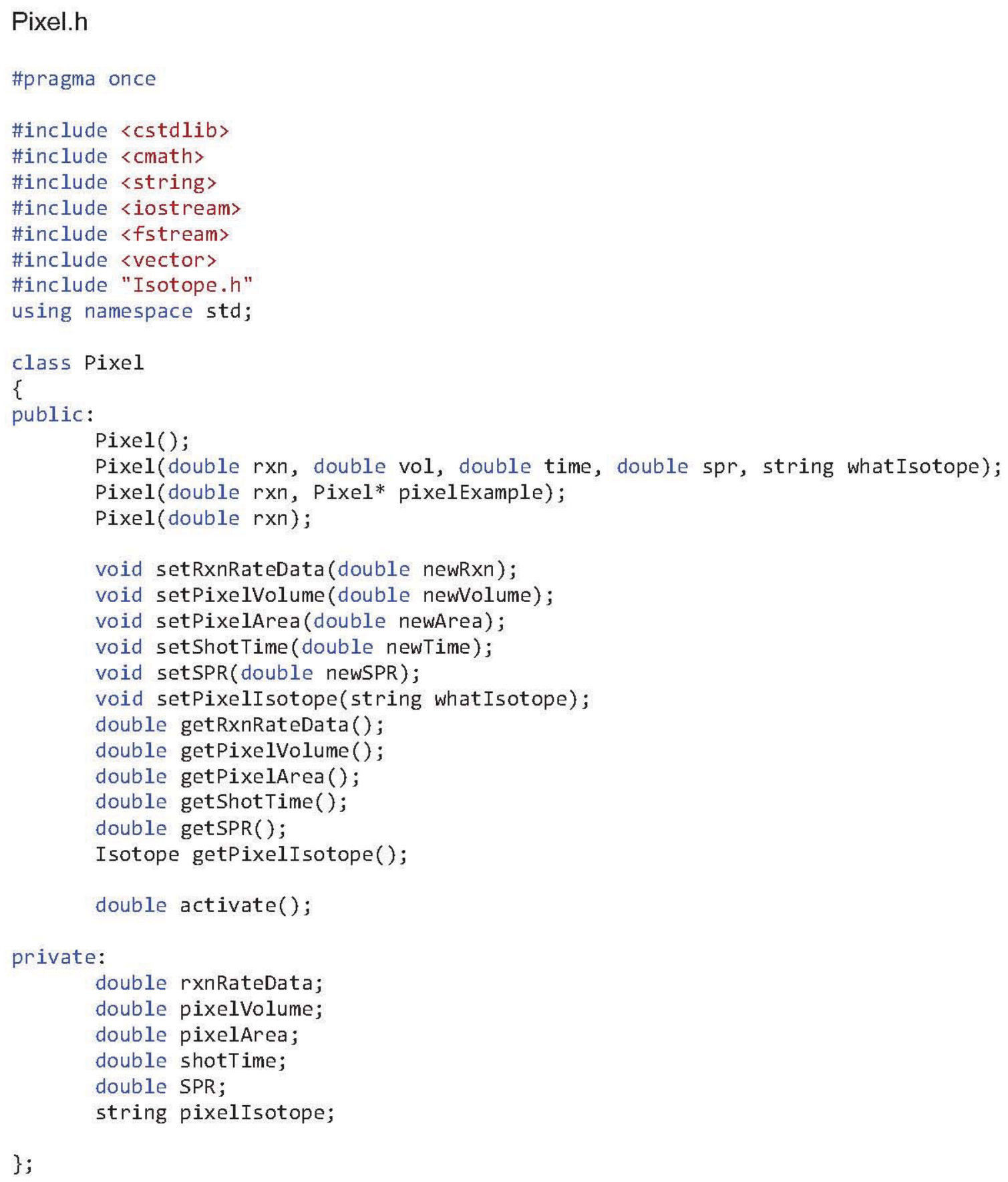




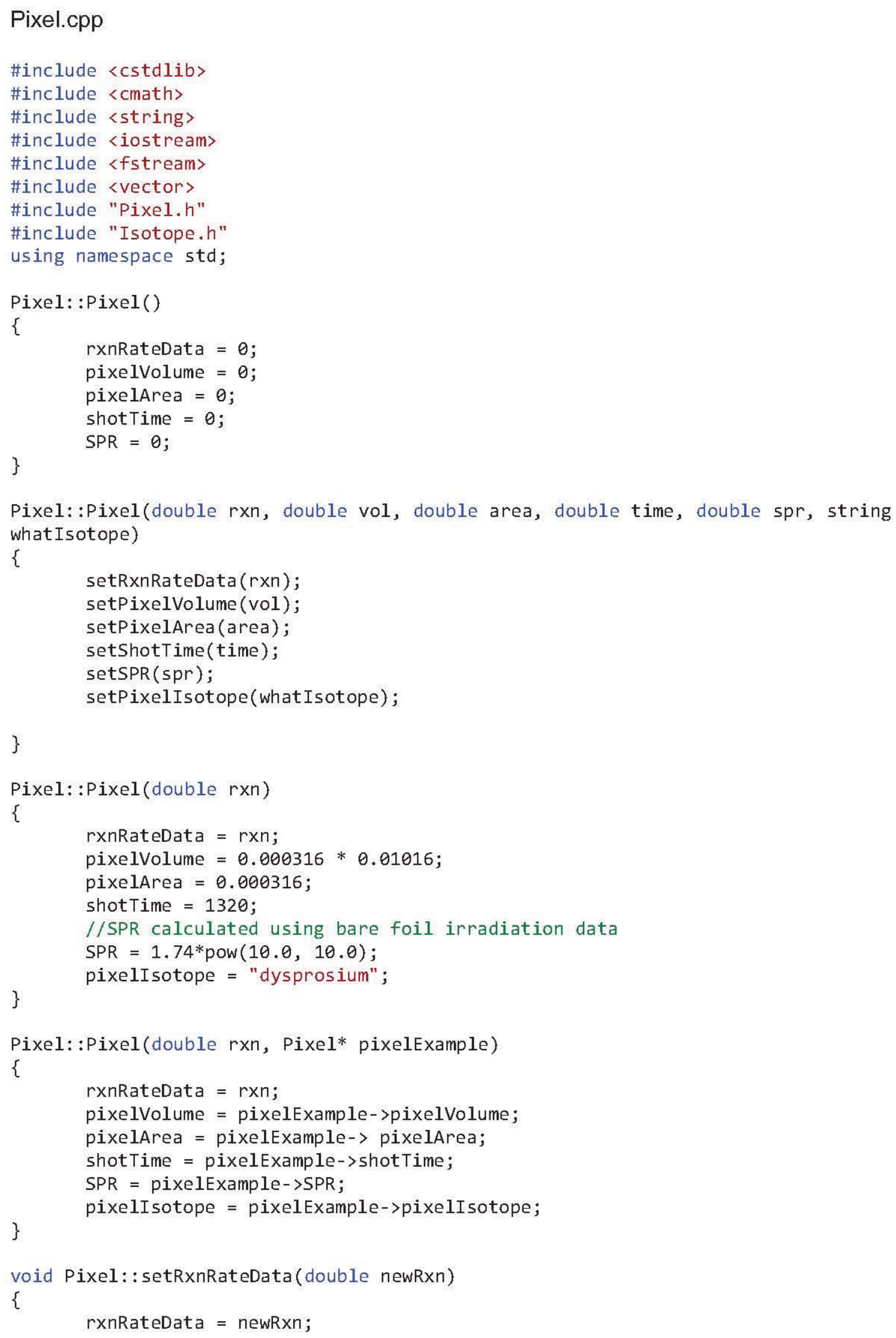




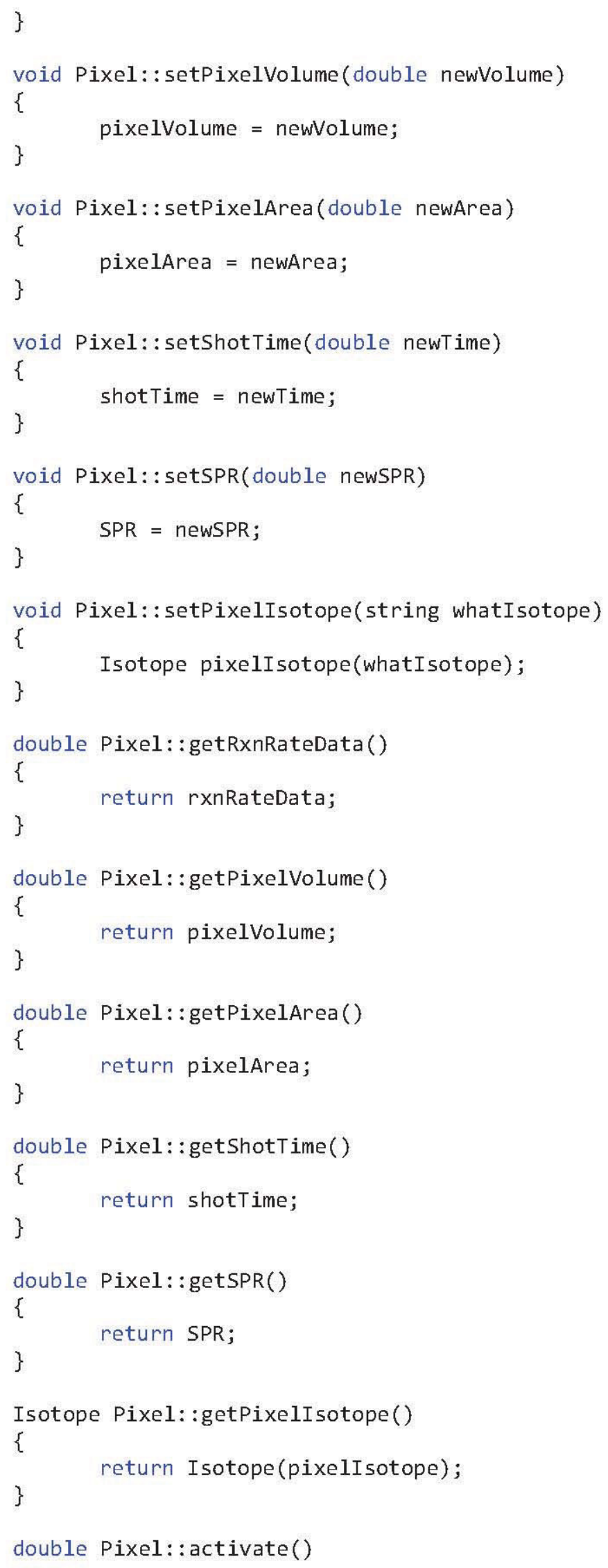


double lambda = getPixelisotope (). calcLambda();

double forDecay $=1-\exp (-$ lambda $*$ shotTime);

double multconsts $=$ pixelVolume $*$ SPR $* \exp (-$ lambda $* 480)$;

double microCuries $=1 /(3.7 * \operatorname{pow}(10.0,10) * \operatorname{pow}(10.0,-6))$;

\}

return rxnRateData * forDecay * multConsts * microcuries; 


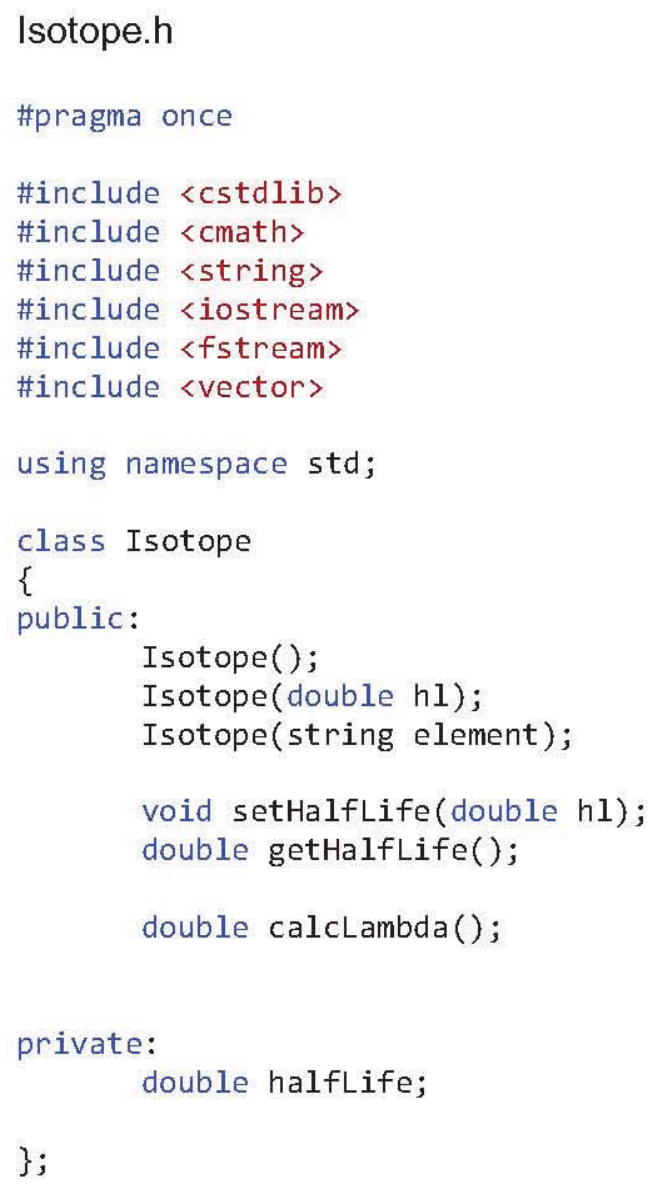




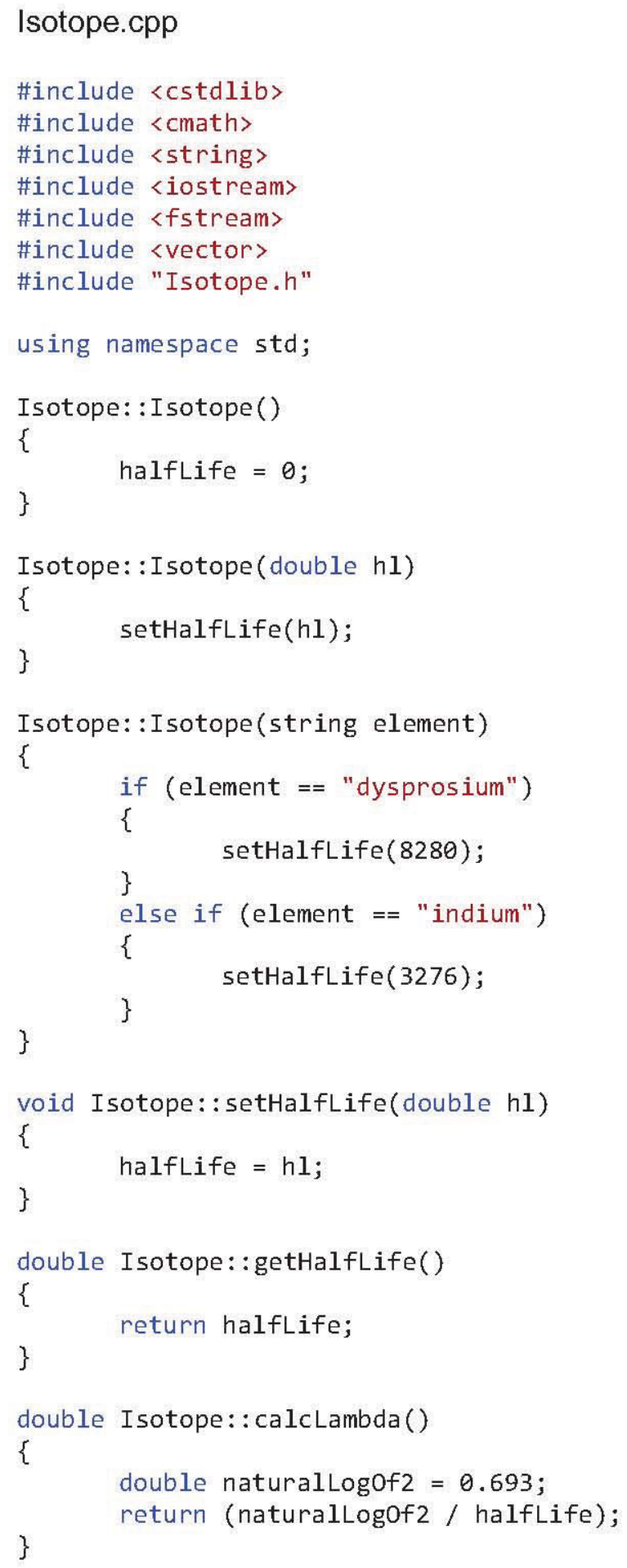

